\title{
SHARED-ENERGY PREDICTION MODEL FOR SHIP-ICE INTERACTIONS
}

\author{
by \\ (C) Amy Price, B.Eng.

\begin{abstract}
A thesis submitted to the School of Graduate Studies
Master of Engineering
\end{abstract} \\ in partial fulfillment of the requirements for the degree of
}

Faculty of Engineering and Applied Science

Memorial University of Newfoundland

October 2021

St. John's

Newfoundland and Labrador

Canada 


\begin{abstract}
To model the structural response of low- and non-ice-class ships requires both the ice and structural responses to be accounted for. A weakly coupled shared-energy algorithm is presented to model a ship-ice interaction for low- and non-ice-class ships. The algorithm is created to model the ship response within the operational design limits of the International Association of Classification Societies (IACS) Polar class rules [1], and therefore, employs many similar assumptions and methods as the IACS Polar rules. First, the Popov collision model [2] is implemented to determine the initial kinetic energy of the system. Daley's energy based ice collision force models [3] are adopted, assuming a pressure-area relationship, to determine the ice-crushing energy. The structural deformation energy is determined by superimposing the frame and plate response. The flexural strain energy of the beam is derived from classical beam theory. The plate deflection energy is modelled with a meta-model created through experiments conducted with finite element analyses and the design of experiments methodology. The algorithm determines the energy distribution through a system of equations that iterates the shared contact force until the internal energy of the system (the ice and structural response) equals the initial kinetic energy of the interaction. Various finite element benchmarking analyses are conducted to assess the prediction capabilities of the individual structural models, the combined structural response, and the algorithm as a whole.
\end{abstract}




\section{Acknowledgements}

First, I would like to acknowledge my gratitude for the financial support from two NSERC research projects: the NSERC / Husky Energy Industrial Research Chair in Safety at Sea; and the NSERC DND/CRD "Operational Capabilities of Low- and Non-ice-class Structures in Ice" grant, with the following project partners: Defence Research and Development Canada (Atlantic), Vard Marine Inc., the American Bureau of Shipping (ABS), and the Newfoundland \& Labrador Provincial Government.

Next, I would like to thank my co-supervisors, Dr. Brian Veitch, and Dr. Bruce W.T. Quinton, for encouraging both my academic and professional development throughout this whole process. Thank you both, for your continued support and patients with me during the writing process and for sharing all your knowledge and experience.

Also, thank you, Dr. Claude Daley, for your many discussions sharing your extensive knowledge and years of experience with ship structures and ice mechanics. Dr. Jennifer Smith, thank you, for helping me improve my technical writing skills with your tutorials and help with any research or administrative related questions. I would also like to thank all the graduate students I interacted and collaborated with from both research projects, for sharing their research expertise, ideas, and professional experiences.

Finally, thank you to my family and friends for always encouraging and supporting me during this process. I could not have completed this thesis without your help and support. 


\section{Table of Contents}

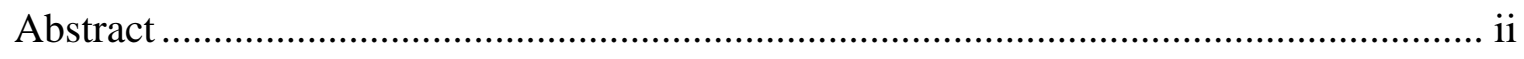

Acknowledgements ........................................................................................... ii

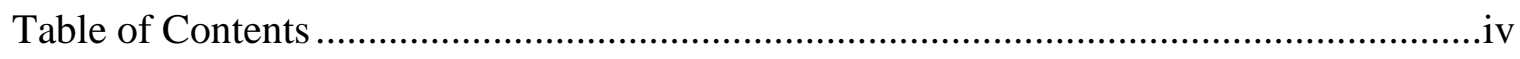

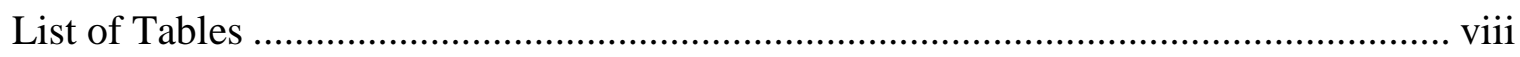

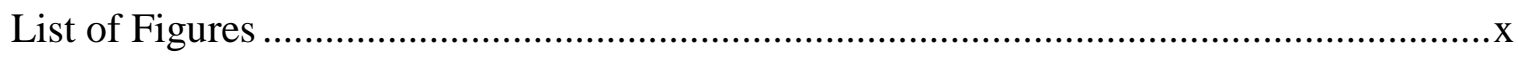

List of Symbols, Nomenclature or Abbreviations ................................................... xiii

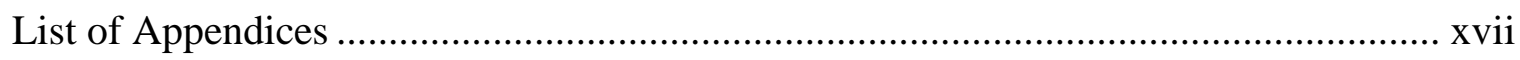

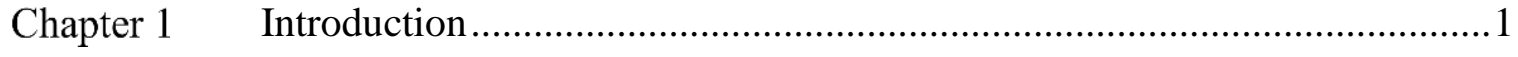

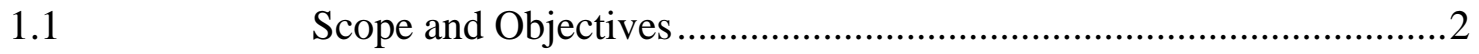

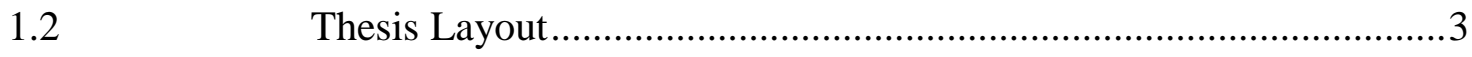

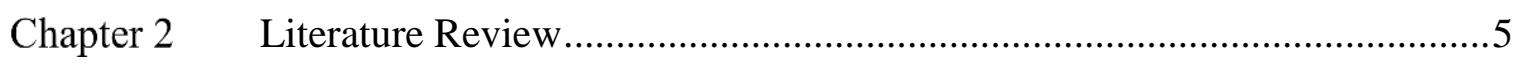

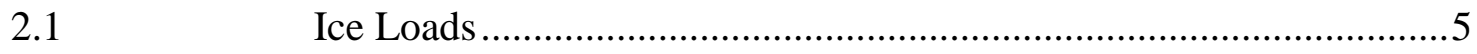

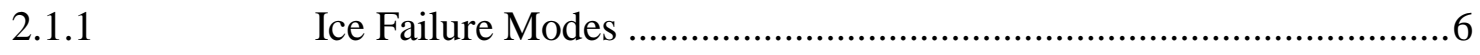

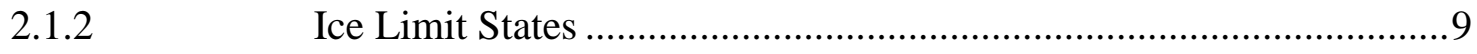

Ship Collison Models.........................................................

Types of Collision Models ................................................... 10

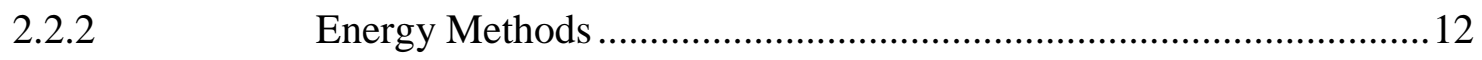


Chapter 3 Model Methodology...... 
3.4.2.3 Creating the Plate Meta-Model 


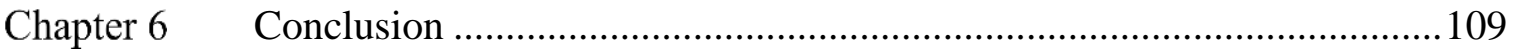

6.1

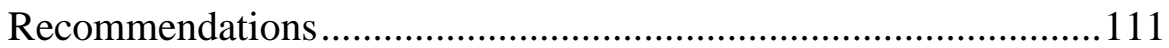

6.2

Future Work

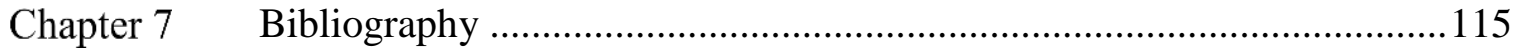

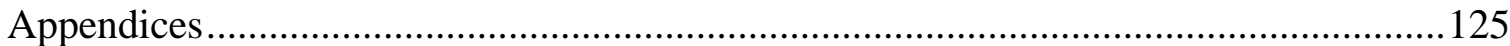




\section{List of Tables}

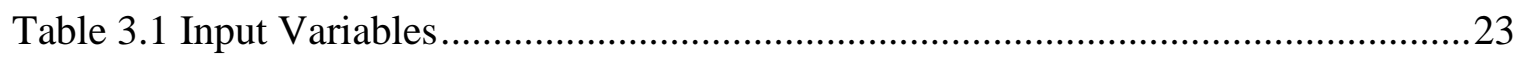

Table 3.2 Loads to Cause Edge and Centre Yield .........................................................50

Table 3.3 Plate Meta-Model Experimental Levels ......................................................50

Table 3.4 Plate Meta-Model Experimental Runs ........................................................51

Table 3.5 ANOVA Analysis of Elastic Plate Model ....................................................54

Table 3.6 Fit Statistic R ${ }^{2}$ Elastic Plate Model ...........................................................55

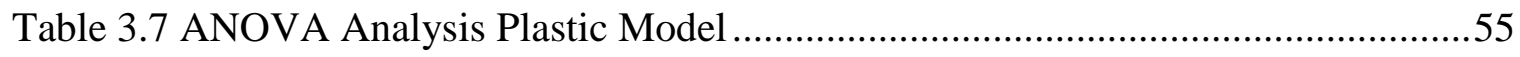

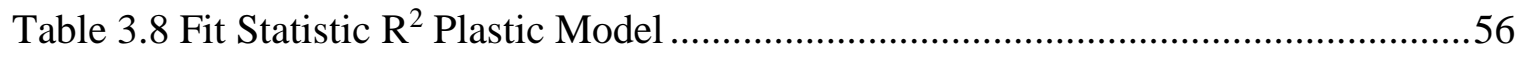

Table 3.9 Plate Transition and Termination Experimental Levels and Runs ..................57

Table 4.1 Beam - Transition and Termination Loads ................................................64

Table 4.2 Beam - Collapse Energy Comparision ...................................................66

Table 4.3 Beam Model Energy Prediciton Statistical Analysis ...................................67

Table 4.4 Elastic Plate FEA Verification Points.................................................... 71

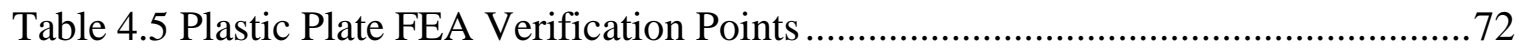

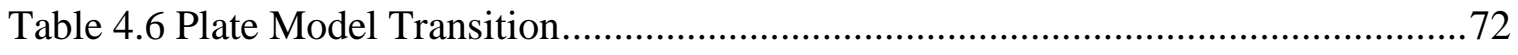

Table 4.7 Plate Termination Energy Prediction ................................................... 73

Table 4.8 Plate Meta-Model Energy Prediction Statistical Analyses .............................74

Table 4.9 Grillage Patch Load - Load at Yield .................................................... 84

Table 4.10 Grillage Patch Load Energy Prediction Statistical Analysis.........................85

Table 4.11 Grillage-Ice - Load at Yield (Plastic Interaction) .....................................95

Table 4.12 Grillage-Ice - Structural Termination Energy ........................................96 
Table 4.13 Grillage-Ice - Plastic Collapse Energy Responses .......................................97

Table 4.14 Grillage-Ice Energy Prediction Statistical Analysis ..................................98 


\section{List of Figures}

Figure 3.1 Ice and Structural Energy Distribution ....................................................21

Figure 3.2 Algorithm General Approach Flowchart .................................................22

Figure 3.3 Wedge Normal to the Hull Contact Geometry [3] ......................................31

Figure 3.4 Wedge Normal to the Hull Contact Geometry Conversion to a Rectangular Patch

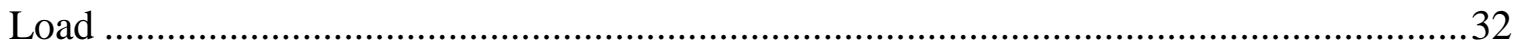

Figure 3.5 Beam Deformation Response Energy...................................................... 35

Figure 3.6 Elastic Beam Boundary and Loading Conditions .......................................36

Figure 3.7 Plastic Beam Boundary and Loading Conditions..........................................38

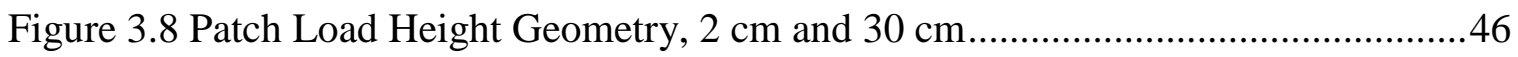

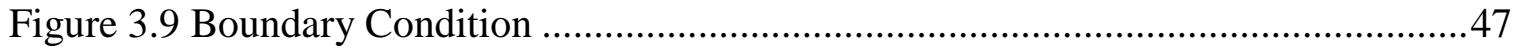

Figure 3.10 Elastic-Plastic Bi-linear Material Response .........................................47

Figure 3.11 Patch Load Loading Condition....................................................................48

Figure 3.12 Plate Models Mesh Convergence Analyses............................................49

Figure 3.13 Normal Plot of Residuals - Elastic Plate Response No Transformation........52

Figure 3.14 Normal Plot of Residuals - Elastic Plate Response Natural Log Transformation

Figure 3.15 Box-Cox Plot - Elastic Plate Response No Transformation .........................52

Figure 3.16 Box-Cox Plot - Elastic Plate Response Natural Log Transformation............52

Figure 3.17 Normal Plot of Residuals - Plastic Plate Response No Transformation........53

Figure 3.18 Normal Plot of Residuals - Plastic Plate Response Natural Log Transformation 
Figure 3.19 Box-Cox Plot - Plastic Plate Response No Transformation .53

Figure 3.20 Box-Cox Plot - Plastic Plate Response Natural Log Transformation .53

Figure 4.1 Beam FEA Model (Half-length patch load) .60

Figure 4.2 Elastic Perfectly Plastic Material Model .61

Figure 4.3 Beam FEA Benchmarking - Mesh Convergence Analyses .62

Figure 4.4 Uniformly Loaded Beam - Energy Analysis .63

Figure 4.5 Beam Half-Length Patch Load - Energy Analysis

Figure 4.6 Beam Quarter-Length Patch Load - Energy Analysis .64

Figure 4.7 Plate Energy Analysis - 27.2 cm Patch Load Height

Figure 4.8 Plate Energy Analysis - $20 \mathrm{~cm}$ Patch Load Height .69

Figure 4.9 Plate Energy Analysis - $12.1 \mathrm{~cm}$ Patch Load Height .70

Figure 4.10 Plate Energy Analysis - $6.7 \mathrm{~cm}$ Patch Load Height .70

Figure 4.11 Grillage Structure - Patch Load on Frame .76

Figure 4.12 Grillage Structure - Loading Condition Between Frames with Fixed Boundary Conditions .76

Figure 4.13 Grillage Structure - Top View Dimensions (In mm) .77

Figure 4.14 Grillage Structure - Left Side View Dimensions (In mm).........................78

Figure 4.15 Grillage Structure Patch Load - Mesh Convergence Analyses......................79

Figure 4.16 Grillage Patch Load - Frame Energy Response ........................................80

Figure 4.17 Grillage Patch Load - Plate Energy Response ........................................81

Figure 4.18 Grillage Patch Load - Full Grillage Energy Response................................83

Figure 4.19 Grillage Patch Load - Energy Prediction Box and Whisker Plot...................86

Figure 4.20 Grillage-Ice Interaction FEA Model .87 


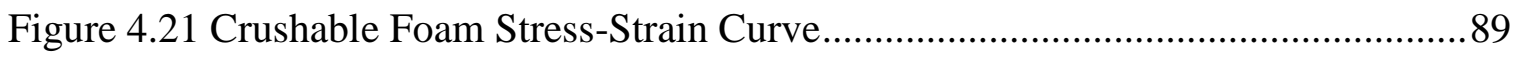

Figure 4.22 Grillage-Ice Interaction Mesh Convergence Analyses ..............................90

Figure 4.23 Elastic Grillage-Ice Interaction Structural Response ...............................91

Figure 4.24 Plastic Grillage-Ice Interaction Structural Response ................................92

Figure 4.25 Grillage-Ice Interaction Plastic Beam Response ....................................93

Figure 4.26 Grillage-Ice Interaction Plastic Plate Response........................................94

Figure 4.27 Grillage-Ice Interaction Plastic Ice Response........................................95

Figure 4.28 Grillage-Ice Energy Prediction Box and Whisker Plot ..............................98

Figure C.1 Beam Boundary and Loading Conditions ............................................ 142

Figure C.2 Elastic Beam Reaction Forces and Moments......................................... 142

Figure C.3 Plastic Beam Reaction Forces and Moments ....................................... 146 


\section{List of Symbols, Nomenclature or Abbreviations}

\begin{tabular}{|c|c|}
\hline ANOVA & Analysis of Variance \\
\hline B.C. & Boundary Conditions \\
\hline DoE & Design of Experiments \\
\hline $\mathrm{FE}$ & Finite Element \\
\hline FEA & Finite Element Analysis \\
\hline IACS & International Association of Classification Societies \\
\hline $\mathrm{PC}$ & Polar Class \\
\hline PL & Patch Load \\
\hline UR & Unified Requirements \\
\hline$\alpha$ & Waterline angle \\
\hline$A$ & Contact force variable in meta-model analysis \\
\hline$A_{c}$ & Contact area \\
\hline$A_{n}$ & Normal contact area between the ship and ice \\
\hline$a$ & Distance to the beam patch load from the start of the beam \\
\hline$A M_{x}$ & Added mass factor in surge \\
\hline$A M_{y}$ & Added mass factor in sway \\
\hline$A M_{z}$ & Added mass factor in heave \\
\hline$A M_{\text {roll }}$ & Added mass factor in roll \\
\hline$A M_{\text {pit }}$ & Added mass factor in pitch \\
\hline$A M_{\text {yaw }}$ & Added mass factor in yaw \\
\hline$A R$ & Aspect Ratio \\
\hline$\beta$ & Frame angle \\
\hline$\beta$, & Normal frame angle \\
\hline$B$ & Patch load height variable in meta-model analysis \\
\hline$B_{\text {ship }}$ & Breadth of ship \\
\hline$b$ & Length of the beam patch load \\
\hline$C_{B}$ & Block coefficient \\
\hline$C_{m}$ & Midship coefficient \\
\hline$C_{o}$ & Effective mass coefficient \\
\hline$C_{w p}$ & Waterplane coefficient \\
\hline$E$ & Young's Modulus \\
\hline$E_{\text {tan }}$ & Tangent Modulus (Slope of the plastic linear material response) \\
\hline$e x$ & Ice-crushing exponent \\
\hline$F$ & Force \\
\hline$F(x)$ & Applied/ Reaction force across the beam \\
\hline
\end{tabular}




$\begin{array}{ll}F_{n} & \text { Contact force } \\ f_{A} & \text { Function of area } \\ f_{a} & \text { Ice-crushing contact geometry term } \\ f_{F} & \text { Function of force } \\ f_{x} & \text { Ice-crushing contact geometry term } \\ \gamma & \text { Shear angle } \\ H & \text { Height (depth) of ship } \\ h_{p l} & \text { Rectangular patch load height } \\ I & \text { Moment of inertial } \\ I E & \text { Ice-crushing energy } \\ K E & \text { Kinetic energy } \\ L_{b} & \text { Length of the beam } \\ L_{b} / 2 & \text { Beam patch load a half-length of beam length } \\ L_{b} / 4 & \text { Beam patch load a quarter-length of beam length } \\ L_{i c e} & \text { Edge length of square ice piece } \\ L_{s h i p} & \text { Overall length of ship } \\ l, m, n & \text { Directional cosine terms } \\ \lambda, \mu, \eta & \text { Moment arm terms } \\ M & \text { Mass } \\ M(x) & \text { Bending moment across the beam } \\ M_{A e} & \text { Elastic reaction moment at the start of the beam } \\ M_{B} & \text { Reaction moment at end of the beam } \\ M_{c} & \text { Moment at the center of the plastic beam } \\ M_{E} & \text { Moment at the ends of the elastic beam } \\ M_{e} & \text { Effective mass } \\ M_{e_{i c e}} & \text { Effective mass of the ice } \\ M_{e_{s h i p}} & \text { Effective mass of the ship } \\ M_{e_{s y s}} & \text { Effective mass of the system } \\ M_{i c e} & \text { Mass of the ice } \\ M_{P} & \text { Plastic moment of the beam } \\ M_{s h i p} & \text { Mass of the ship } \\ \varphi & \text { Ice-wedge angle } \\ P & \text { Contact point between structure and ice } \\ P_{a v e} & \text { Average pressure } \\ P_{o} & \text { Nominal ice pressure of } 1 \text { m } \\ Q(x) & \text { Shear force across the beam } \\ R_{A e} & \\ R_{A p} & \text { Elastic reaction force at the start of the beam } \\ & \end{array}$




$\begin{array}{ll}R_{B} & \text { Reaction force at end of the beam } \\ r_{x}{ }^{2} & \text { Squared mass radii of gyration in roll } \\ r_{y}{ }^{2} & \text { Squared mass radii of gyration in pitch } \\ r_{z}{ }^{2} & \text { Squared mass radii of gyration in yaw } \\ \sigma_{y} & \text { Yield stress } \\ S E & \text { Structural response energy } \\ S E_{b_{p}} & \text { Plastic beam response energy } \\ S E_{p_{e}} & \text { Elastic plate response energy } \\ S E_{p_{p}} & \text { Plastic plate response energy } \\ \Delta \theta & \text { Change in slope of beam } \\ \theta(x) & \text { Slope across the length of the beam } \\ T & \text { Draft of ship } \\ t & \text { Beam cross-section thickness } \\ U & \text { Beam flexure strain energy due to bending } \\ U_{e} & \text { Elastic beam flexure energy } \\ U_{p} & \text { Plastic beam flexure energy } \\ u_{n} & \text { Distributed beam patch load } \\ V_{n} & \text { Relative velocity between ship and ice } \\ v(x) & \text { Deflection across the length of the beam } \\ W_{R} & \text { Rotational Work } \\ W_{z} & \text { Triangular width of the contact geometry } \\ w & \text { Beam cross-section width } \\ w_{n o m} & \text { Nominal rectangular patch load width } \\ w_{p l} & \text { Rectangular patch load width } \\ Y_{E d} & \text { Load to cause yield at the edge of the plate } \\ Y_{C H} & \text { Load to cause yield at the centre of the plate } \\ y & \text { Cross-sectional area distance to the neutral axis } \\ \ddot{\zeta} & \text { Net normal acceleration at the point of contact } \\ \zeta_{n} & \text { Normal ice indentation } \\ Z & \text { Plastic section modulus } \\ \mathrm{Kg} & \text { Kilogram } \\ \mathrm{Kg} / \mathrm{m}^{3} & \text { Kilogram per Cubic Meter } \\ \mathrm{J} & \text { Moule } \\ \mathrm{m} & \text { Cetentimetre } \\ \mathrm{cm} & \mathrm{mm} \\ \mathrm{m} / \mathrm{s} & \end{array}$




$\begin{array}{ll}\mathrm{N} & \text { Newton } \\ \mathrm{kN} & \text { Kilonewton } \\ \mathrm{kN} / \mathrm{m} & \text { Kilonewton per Meter } \\ \mathrm{Pa} & \text { Pascal } \\ \mathrm{MPa} & \text { Mega-Pascal } \\ \mathrm{GPa} & \text { Giga-Pascal } \\ \circ & \text { Degree } \\ \mathrm{rad} & \text { Radian }\end{array}$




\section{List of Appendices}

Appendix A - Algorithm (MATLAB Script) .................................................... 126

Appendix B - Popov Model Description ............................................................. 137

Appendix C - Beam Patch Load Derivation............................................................ 142

Appendix D - Beam Strain Energy Integration (MATLAB Script) .............................149 


\section{Chapter 1 Introduction}

Ship-ice interaction research has focused on the interaction between icebreakers and ice, but assumptions and methods typically used with icebreakers are less applicable to lower and non-ice-class ships. Hence, with the growing interest in the operational capabilities of low- and non-ice-class ships, a comprehensive understanding and model of the structural response of these ships within their standard operational ice loads is beneficial for both designers and operators alike.

Current ship-ice interaction models, especially with icebreakers, assume that all the collision energy produced during the interaction goes into ice-crushing. From there, the structural design load is extrapolated from the load produced during the peak ice-crushing response. With low- and non-ice-class ships, this assumption of all the energy going into ice-crushing is less valid, since with lower ice-class ships both the structural and icecrushing responses are significant in dissipating the energy of the collision. In addition, the assumption that the energy is absorbed during ice-crushing artificially increases the structural design load. This assumption is more valid with stiffer structures like higher iceclass ships, but prevents the full capabilities of less stiff structures, like low- and non-iceclass ships, from being modelled. By distributing the collision energy between the structure and ice, this reduces the contact force by having two bodies absorb portions of the impact energy compared to a single body responding to the full collision energy. Therefore, a versatile ship-ice interaction algorithm is developed, hereby accounting for the structural response of the ship, through the inclusion of both the ship structural response and icecrushing models. 


\subsection{Scope and Objectives}

The shared-energy algorithm models the structural response of a ship-ice interaction up to the allowable International Association of Classification Societies (IACS) Unified Requirements (UR) for Polar Class (PC) rules limit states of plastic 3-hinge collapse. Thus, the ship response anywhere within the allowable elastic and plastic response range can be found. From there, the ship response is interpreted as safe (within the elastic response) or within a cautionary response range (a plastic structural response up to the limit state); the algorithm also identifies if the response is dangerous (beyond the allowable limit state). The nature of the structural model also allows for simple adaptation to various structural arrangements since the structural components are analyzed individually and with models that only require basic geometric parameters.

Energy methods are used with this approach to equate the total energy of the collision, found with the Popov method [2], to the energy distributed between the ice-crushing and structural response. The progressive response of the ice and structure is linked through the shared contact force of the interacting bodies and iterated until the combined ice-structure response equals the total kinetic energy of the collision. For the ice-crushing energy, Daley's energy based ice collision force model [3] developed with the pressure-area relationship is adapted for this algorithm. The ship structure is broken into individual grillage components: plates and beams. The bending energy of the beam is derived as the flexural strain energy, using classical beam theory. The plate membrane and bending energy is modelled with a meta-model developed with a design of experiments uniform design approach. 
As the structural models are developed, they are individually benchmarked using finite element analysis. The completed algorithm is then benchmarked against a finite element ship grillage, through both a fixed patch load and an ice floe interaction.

\subsection{Thesis Layout}

The rest of this thesis examines the current state of knowledge, the developed algorithm, the benchmarking process, and a final discussion of the proposed algorithm.

Chapter 2 starts by looking at the current understanding of ice and how it is modelled during a collision. From there, ship collision models are assessed, both with ice and with other ships or structures, concentrating on how the structural response is modelled. Finally, the design of ship structures for operations in ice is examined.

Chapter 3 focuses on the development of the shared-energy algorithm. The chapter starts by giving a general description of how the algorithm is setup. The rest of the chapter discusses the individual models used in the algorithm, including the kinetic energy model, ice-crushing model, and structural response models.

In Chapter 4, various types of benchmarking analyses are conducted to assess the applicability of the developed algorithm. The benchmarking starts with an analysis of the individual structural components of the grillage with finite element analyses (FEA). Next, an FEA grillage structure is compared to the algorithm, with two types of loading scenarios. The FEA grillage experiences a fixed patch load for one loading event and an ice piece interaction for the other. 
Chapter 5 discusses the development of the algorithm and conclusions drawn during the benchmarking phase. Finally, in Chapter 6, the conclusion, potential future work, and recommendations are considered. 


\section{Chapter 2 Literature Review}

A ship-ice interaction can be broken up into three components: ice response, ship response, and the interface between the ship and the ice [4]. The first section of this literature review examines the general knowledge about the ice response and interface. The second part of this chapter investigates the ship response of more generic collision models, in addition to more specific ship-ice collision models.

\subsection{Ice Loads}

Ice-induced loads on a ship can be categorized as global or local loads. Both global and local loads interact with the structure, but it is the effect of the interaction that is distinguished as global or local. Ice loads can be generated during level ice-breaking operations and individual loading events from ice floes. The effects of global loads concern the overall strength and stability of a ship. Local loads occur over a more defined region of the ship hull and are typically used to determine the structural design of a ship hull and strength requirements for the ship's structural elements $[5,6]$.

An ice-breaking event can be divided into a five-step process, applicable with either level ice or ice floes [7]. First, the ice interacts with the structure through local ice-crushing. If the ice piece has a large enough surface area, the ice will fail in flexure and break off an ice piece. Then the new ice piece or the original ice piece gets pushed downward and submerged under or along the side of the ship. Finally, the broken ice pieces slide along the hull of the ship until they resurface again [7]. Typically, the maximum ice load arises at the end of the ice-crushing period, which can occur just before the ice fails due to bending, or 
when the ship stops after all its kinetic energy is expelled in ice-crushing, or if the ice floe glances away [7]. Therefore, to try and reduce the loads on the hull, ships that are designed to transit in ice, like icebreakers, have hulls designed to induce bending failure in the ice [8].

During the ice-crushing process, initially, the sharp local protrusions of the ice spall. Once the easily crushable edges spall, the contact area starts to grow, and the structure starts to experience elastic deformation [9]. Ice also does not produce a uniform pressure during icecrushing but generates a more randomly developing spatial distribution with varying sporadic peak forces $[10,11]$. These high-pressure zones randomly appear across the contact area depending on the ice properties, loading geometry and build-up of ice pressure. The change in force and location of these high-pressure zones is due to the accumulation of ice pressure prompting eventual spalling events, which release the ice pressure by discharging the buildup of pressure in the intact zone. With the release of one high-pressure zone, a new one is formed elsewhere, and the process repeats $[12,13]$.

\subsubsection{Ice Failure Modes}

Ice failure modes can be broken down into two basic groups: ice-crushing and bending. Depending on the failure mode and loading rate, this affects the ice pressure [12]. Therefore, due to the various modes in which ice may fail, holistic ice response models, like Kim et al. [14], are required to account for the multiple ice failure responses that may occur during an ice interaction. In cases with relatively thin ice, the dominant failure mode is bending failure [7]. With slightly larger or more confined ice floes, typically the ice will 
experience some crushing before failing in bending. Smaller-sized ice floes tend to rotate with no significant ice edge crushing [14].

Ice-crushing occurs over an area and is typically modelled as a contact pressure instead of a contact force [15]. Ice exerts pressure through both direct contact and a layer of extruded crushed ice material [16]. Solid direct contact produces the highest pressure, even when the ice is starting to experience internal damage and cracks, since the ice is still able to sustain high pressure due to high confinement at the center of the contact area [16]. The shape of the contact area and indenter can affect the flow of crushed ice. If restricted, the crushed ice can result in increased confinement and contact pressure. Likewise, if the crushed ice is allowed to extrude and not accumulate, this decreases the contact pressure [15, 17]. Depending on the loading rate, crushed ice behaviour can be ductile or brittle [6, 15]. At slower loading rates, the ice has a ductile response and a more uniform pressure. As the loading rate increases, the ice becomes more brittle, resulting in more sporadic peaks in pressure and causing more splitting and shattering of the ice $[9,15]$.

Different ice-crushing models have been proposed over the years, but the pressure-area relationship is by far the most commonly used. The pressure-area relationship simplifies the complex nature of ice-crushing, by creating an empirical model where the ice-crushing loads vary with the change in nominal contact area [7]. Kurdjumov and Kheisin's hydrodynamic model is also commonly referenced in the literature $[8,15,18,19]$, which assumes a thin layer of crushed ice between the structure and the ice. However, it is often debated that the empirical evidence does not support the assumptions of the hydrodynamic model. Also, due to the Reynolds thin-film equation, the formula is too complicated to be 
solved explicitly, requiring a numerical integration, so the hydrodynamic model is often passed up for simpler models $[8,15]$. During a review of the ice-crushing failure process, Riska [15] also noted a couple of other less common models: the Korzhavin ice pressure model and the Winkler foundation model.

Even though the pressure-area relationship is the most commonly used ice-crushing model that does not mean that it does not come with its issues. The pressure-area model uses empirical data to produce a scaling effect of the geometric area [15]. Most of the empirical data employed with the model was collected through strain gauges attached to ship hull structural elements, which does not allow for very good spatial resolution and suffers from cross-talk between frames, affecting both the spatial resolution and enhancing errors [10]. In part due to the poor spatial resolution of the strain gauge data, the pressure-area relationship traditionally assumes a uniform pressure across the whole contact area [15], but more recent research suggests that a "spatial" pressure-area model more realistically describes the local peak pressures in relation to the total contact area [16]. The pressurearea relationship also assumes that the average pressure will decrease as the nominal contact area increases [16, 19]. Timco and Sudom [12] analyzed this assumption and found that the pressure does decrease with increased area, but not always. Due to the nature of the ice-crushing experiments conducted, the pressure-area relationship appears to have a false plateau. The trend in the pressure-area experimental data of decreasing pressure with the increase of area is likely influenced by the experimental set-ups through either the limiting force capabilities of the ram, or the limited momentum or energy of a ramming vessel, as the interaction can only consume the maximum energy introduced into the system [16]. 


\subsubsection{Ice Limit States}

Limit state methodology is the association between the structural capability or resistance, and the internal and external actions. With this methodology, the structural resistance must be greater than the action, with the maximum design load at the point where the resistance equals the action. There are 3 types of actions: permanent action, for example, the weight distribution of the structures; variable action, for example, any of the different types of loading scenarios; and environmental action, for example, ice, waves, and wind [20].

With ship-ice interactions, the ice environmental actions can include any of the three following limit states: ice strength, the driving force, and momentum [21]. If an ice interaction has unlimited driving force and momentum, the ice will consume the collision energy through ice-crushing unless interrupted by either a lower energy ice failure, like bending in flexure or radial cracking, or enough energy is inputted into the interaction to flip or rotate the ice away from the structure. Otherwise, the interaction will be limited by either the driving force or momentum. The driving force of the interaction refers to the external force propelling the ice towards the ship or structure, for example, wind, current, or the growth of landfast ice. The momentum of interaction can also be a limit state. Depending on the speed of the ship, the internal response energy can not exceed the amount of energy put into the system. Therefore, once all the initial energy of the interaction from both the ship and the ice is expelled, the interaction has reached its peak possible load.

\subsection{Ship Collison Models}

Ship damage can be split into three different categories: collisions, groundings, and contact. Ice can produce either collision or contact damage [22]. Collisions are interactions with an 
initial kinetic energy where the ship has speed and mass at the moment of impact. Grounding damage occurs when a ship strikes the seabed. Contact damage consists of an interaction with a stationary ship or structure, for example, either an ice floe or landfast ice could produce a contact interaction [22]. The rest of the chapter focuses on ship collision damage models.

\subsubsection{Types of Collision Models}

In the literature, ship collision models are typically divided into 5 different categories: empirical and experimental models, finite element based models, analytical models, hybrid models, and probabilistic models [22]. Empirical or experimental models either use existing experimental data or create models with data generated from specified experiments $[17,23$, 24]. Experimental models have been dropping in popularity [22], likely due to cost and time to set up experiments, whereas numerical and finite element models have been increasing in terms of use $[22,24]$. One of the first and well-known empirical models was created by Minorsky [23] to model energy absorption during a collision, based on the volume of damage sustained during the interaction. The model was developed by studying 26 collision cases [25]. Dedicated experimental models gradually overtook empirical models created from observed or deduced trends in experimental datasets, for example, full-scale and model tests are employed to validate the empirically and analytically developed models [22].

With more modern computing power, numerical and finite element models are starting to become the more popular modelling approach $[22,26]$. Both a positive and a negative of FEA models is that more details are required to model the interaction [22], thus also 
producing a more detailed response. If a very specific interaction is being modelled, and the details of the interaction are known, this can be a more accurate approach, since fewer simplifying assumptions are required to model the interaction [27]. FEA models are often used to model the coupling effect of two interacting bodies, with either ice or structural interactions, since both responses and effects of the interaction can be modelled simultaneously [28, 29, 30, 31]. The primary issue with modelling ice in finite element analyses is the lack of experimental data to validate the ice models [17].

Analytical models are the most common models and are often used during the preliminary design phase, structural optimization, and risk assessment, due to their simplicity, speed, and low resource cost $[22,32]$. These models tend to consider plastic analysis and failure, by describing the material and structural failure through the internal and external mechanics of a collision [33, 34, 35]. Analytical models typically apply energy methods and momentum conservation to determine the structural response of a collision [22, 31, 36, 37].

Hybrid-analytical models have started to become more popular in recent years. These models typically combine the simplicity and speed of analytical models with the more detailed numerical approaches, like the finite element method [22,33]. One example is a simplified shared-energy approach presented by Daley and Kim [38], where the localized plastic response is modelled linearly, and the linear response, depending on the structural arrangement, is determined with FEA and the design of experiments methodology. The more standard hybrid models use analytical energy methods to determine the kinetic energy of the interaction and employ FEA to model the structural response of the loading condition $[32,39,40]$. 
The final type of collision model, and probably the least common approach in the literature [31], is the probabilistic model. This model uses available data to predict the type and frequency of damage a vessel experiences during its planned operational use [22]. This approach is also a good way to integrate various scales of an interaction that can lead to a specific collision scenario, such as the operational environment, the ice conditions and properties, ice failure modes and location of impact on the ship hull [5].

\subsubsection{Energy Methods}

There are three different ways to model how the energy is distributed during a collision with energy methods: strength design, ductile design, and shared-energy design [9, 31, 37]. A strength design, which is historically used to model ice collisions, assumes that the ice will dissipate the majority of the collision energy, allowing the structure to be modelled rigidly. The ductile design method assumes that the structure absorbs all the collision energy, and the ice or other interacting body is modelled as infinitely rigid, this approach is more often used while modelling two ships colliding with each other $[33,34,41]$. The final approach, the shared-energy method, is the most realistic, with studies showing that the striking ship can absorb between $20 \%$ and $40 \%$ of the collision energy [25], and during a ship-ice collision the ice can dissipate about $70-80 \%$ of the collision energy with the structure dissipating between $20-30 \%$ of the energy [17]. The shared-energy method is also the most challenging technique to model, due to the additional information required to model the mutually dependent response of the ice and structure [9].

To overcome the mutual dependency of the interaction, there are a few different approaches to modelling the coupled effect of a shared-energy model. A direct approach models the 
structural deformation with fluid-structure interaction techniques. With an indirect approach, a portion of the kinetic energy is dissipated through the relative motion of the ship-ice interaction, modelled as the outer mechanics, the other portion of the energy is consumed through the deformation of the ship and ice, modelled as internal mechanics [31].

There are also two different approaches to model an indirect approach, with either an integrated (or fully coupled) approach and a segregated (or weakly coupled) approach [29, 31]. With an integrated approach, non-linear finite element methods are required to model the internal energy dissipation of the interaction. A weakly coupled interaction is more of a simplified analytical approach, which integrates the response and relative displacement in reference to each other at each time step, assuming the ice and structure respond separately [29, 31].

\subsubsection{Ice Collisions}

The various models discussed in section 2.2.1 were created to model either ice or ship collisions, but very few of those models were created with the intention to model a light ship structure interaction with ice. With more interest recently in understanding the capabilities of existing lighter structural strength ships in ice, more research is still needed in both modelling the structural response, in addition to the ice response, since the assumption previously applied with heavy icebreakers are not valid for lighter weight structures [42]. Also, there has been a lot more focus on the modelling and failure methods of ships transiting in level ice, compared to the modelling and study of an interaction between a ship structure and an ice piece, which is the type of interaction more typical of lower and non-ice-class ships [14]. The quality of the model will also be affected by the 
general knowledge of the subject, which is part of the reason why there is a lack of more detailed and accepted ice models [17, 22, 42]. The biggest difference with lightweight ship structure response in ice compared to icebreakers is the increased structural response that is not typically accounted for with traditional ship-ice collision models, as mentioned in the Energy Methods section. In addition, the shared-energy approach is not as well studied, with the lack of experiments suggesting either a lack of interest or difficulty setting up shared-energy type experiments [9]. Nonetheless, the following sections examine the international Polar Class standards and two common ice interaction models that are also adopted into the Polar Class rules.

\subsubsection{IACS PC Rules}

The International Association of Classification Societies (IACS) Polar Class (PC) Unified Requirements (UR) are a set of international ice-class rules that were published in 2006 to unify the various regional ice-class rules [1]. The ice-class rules were created as a construction standard for the design of ships operating in ice-covered waters, and therefore do not necessarily represent the full capabilities and limitations of the vessel, depending on the type of ice conditions and operations [8].

IACS PC structural requirements are based on a glancing bow impact with a thick edge infinite mass ice piece $[19,43]$, which induces a simple quasi-static flexural limit force that is not valid with thinner ice and higher speed impacts [8]. The ship-ice interaction model is created assuming that the ice fails in crushing, and the ice-crushing forces can not exceed the force required to cause the ice to fail in bending [19]. The design loads are governed by the kinetic energy of the interaction determined with a Popov-based loading event [2]. By 
assuming a Popov loading event, the collision is reduced from a six degree of freedom system to a single degree of freedom. This approach also assumes that all the kinetic energy goes into ice-crushing [19].

The structural requirements for the IACS Polar rules can be considered semi-empirical as many assumptions are determined empirically [44], particularly for the ice design load. To determine the ice load, all the factors are simplified as constants depending on the PC of the vessel. Three of the ice-related parameters in the IACS rules, pressure-area exponent, ice strength factor, and spalling characteristic, are noted to be derived from operational experience with added safety factors, but from a sensitivity analysis, these parameters are found to be the most important assumptions [19]. In addition, the contact geometry has been found to have a strong influence on the ice load, but that is too difficult to predict. Therefore, the UR assumes a wedge shape contact geometry [44] that is contained in the class factors, along with the designated ship speed, ice thickness, and ice strength. The rest of the design load parameters are based on the mechanical properties, vessel geometry, and displacement of the vessel [19].

The IACS rules are created with a plastic limit state, to allow for some plastic behaviour while still maintaining a reserve against actual collapse or rupture [45]. The plastic limit state of the Polar rules assumes a loading condition comparable to 3-hinge collapse, which is considered sophisticated, but still has numerous simplifications, like ignoring the effects of membrane stress and strain hardening [10], which therefore assumes the structure is only experiencing pure bending [46]. The structural requirements for both the frame and plate are derived in isolation, creating a conservative estimation of the capacity of the structural 
elements [14, 47], which from both FEA and experimental assessments suggest, can support 5-10 times the IACS design load [8]. The onset of the plastic material response, of the plate and frame elements, is modelled with the formation of plastic hinges [43]. A plastic hinge model assumes either an elastic perfectly plastic or rigid perfectly plastic material model, where once a hinge forms that section of the structural component takes on the perfectly plastic mechanical properties and can no longer carry load. Nonetheless, the structural element can still generate resistance from the plastic moments, as all the plastic deformation is modelled through the hinge. The plastic hinge limit load is reached once there are a sufficient number of hinges to create a collapse mechanism [46, 48].

For the plate plastic hinge model, the rigid parts are connected through rectilinear plastic hinges [49], with all the plastic deformation occurring through the hinges with the assumption of a rigid-perfectly plastic material model [48]. With the use of rectilinear hinges, the membrane effects of the plate are removed. Modelling the plate response with plastic hinges allows for several possible hinge failure locations and configurations. The IACS rules assume a traditional "roof-top" style collapse pattern [49]. Both the rectilinear hinge model and "roof-top" style collapse pattern produce an overestimation of the plate's capacity, but in the IACS rules the solution is slightly adjusted to produce a more conservative required plate thickness [43, 49].

Similar to the plate criteria, the framing requirements followed the plastic hinge assumptions, which presumes rigid plastic behaviour that ignores large strains and large deflection effects. The framing criteria assessed two different loading conditions to examine two key failure mechanisms, 3-hinge collapse and shear panel collapse, by 
checking the acceptable shear area and section modulus through a central loading and an asymmetrical end loading case [45]. To determine the plastic section modulus, the frame is assumed to be attached to the plate, modelled as a flange. For the central loading condition, a reduced plastic section modulus is assumed at the ends of the frame, since, as the shear load increases at the ends of the frame, the web starts to reduce its ability to contribute to the bending moment $[45,46]$.

\subsubsection{Popov Collision Method}

Popov's collision model [2] is one of the earliest analytical models [4], but is still one of the main approaches used to model ice-induced loads [7]. A Popov collision model neglects damping and buoyancy to simplify a two-body collision into a single degree of freedom system through a single impact impulse value. The kinetic energy normal to the impact is used to determine the contact force described by a simple penetration model of the ice normal to the impact [4]. The inelastic Popov collision assumes that the kinetic energy of the ship will be used to move the ice floe and partially used in crushing the ice edge [2]. A modern example of where a Popov collision is implemented to determine ice loads is the IACS Polar rules [43].

To reduce a collision down to a single degree of freedom, a reduced mass term is found to reduce the interaction down to a single point normal to the hull through a force and a unit acceleration. The movement of each impacting body is modelled through the center of gravity, in addition to rotating about the momentary axis [2]. To uncouple the interaction, the impact is assumed to be transient, and the friction, damping and buoyancy terms are neglected [4]. The ship is modelled as a solid body, with a flat sloped interaction surface. 
The ship structure is modelled as a rigid body, ignoring the elastic strain and yield of the ship [2]. The circular ice floe is assumed immobile, which Popov modelled as a thin plate, with a small thickness relative to its expanse $[2,4]$. The elastic strain in the ice is ignored and the magnitude of the ice pressure will depend on the contact geometry and depth of penetration [2].

Vessel motion other than normal to the impact is ignored, and translations during impact are considered small. Therefore, any change in velocity is a result of the collision, and the reduced velocity is a projection of the ship's normal velocity relative to the impact [2]. The hydrodynamic and static aspects of the impact, such as the water resistance created by the translation of the ship and ice, are considered small relative to the contact stress and are thus lumped in the added mass term [2,4].

\subsubsection{Daley's Energy Based Ice Collision Forces}

Daley's ice collision force model [3] is designed to be applied with two different types of collision models: normal (Popov) and beaching impacts. With these types of collision models, the energy can be expelled in one of two ways through irrecoverable ice-crushing energy and recoverable potential energy. The ice collision force is determined by employing the pressure-area relationship to derive a force-indentation relationship, making the force dependent on the indentation and nominal contact area. With the ice force dependent on the indentation depth either directly or indirectly through the contact area [4], the collision geometry is significant, which therefore requires more detailed equations depending on the type of contact. Daley derived various contact geometries associated with 
the energy based ice collision model [3]. From there the projected normal contact area is used to determine the normal force and indentation.

Even though this model is widely accepted, there are a few limitations with the model, particularly due to the use of the pressure-area relationship to model the ice response. A few different discussions noted that models like the pressure-area relationship ignore other more important factors like ice types, loading rate, aspect ratio, and ice failure mode that can sometimes be more important in determining the ice force than the contact area [12, 19]. The pressure-area relationship also assumes that interaction has a constant velocity, which is not necessarily true, and affects how the response is modelled. At the beginning of the interaction with the initial 'high' velocity, the ice exhibits brittle behaviour, but as that velocity reduces as the interaction consumes the kinetic energy, the ice starts to have a more ductile response, with the crushing pressure at the end of the interaction almost constant and independent of the area [19]. Kim and Amdahl [19] also found during a sensitivity study that the ice spalling effect is the most important parameter in determining ice pressure, but is also the most uncertain parameter, with little validation behind the set value in the IACS PC rules. These issues with the pressure-area relationship are also compounded with the more general difficulties with measuring ice strength in a controlled manner [12]. 


\section{Chapter 3 Model Methodology}

\subsection{General Approach}

As the algorithm is created with potential applications with marine simulators or design assessment tools in mind, a real-time hybrid-analytical approach is the most practical method. With this approach, the algorithm conducts a quick assessment of the ship-ice interaction by estimating the ice and structural response of the collision and can notify the user of the level of damage to the structure as the interaction occurs. The majority of the algorithm implements analytical models, except for the plate model, where a meta-model is created to replace more complicated analytical response models.

To create a structural response model that accounts for both the ice-crushing and structural deformation, the foundation of the algorithm uses energy methods to balance the internal and external energies of the collision. First, during a ship-ice interaction scenario, the algorithm determines the total kinetic energy $(K E)$ of the collision, with the known ice mass, ice velocity, ship mass, ship velocity, and general ship structure parameters. From there, the total kinetic energy, along with the response energies of the ice $(I E)$ and structure $(S E)$ from the collision form a system of equations. Therefore, this algorithm is designed to determine the kinetic energy (the external energy) and to appropriately distribute the kinetic energy produced during the collision between the ice-crushing energy and structural response energy of both the plate and frame (the internal response energies).

Energy models are established dependent on the applied contact force $\left(F_{n}\right)$ for the ice and structure and then placed into the system of equations. This leaves the only unknown in the 
system of equations (Eq. 1) as the contact force between the ice and structure in both the ice and structural energy equations since the force at the point of contact between the ice and structure has to be equal.

$$
K E=I E\left(F_{n}\right)+S E\left(F_{n}\right)
$$

Due to the non-linear nature of the system of equations, a simplified equation solving for the contact force cannot be arranged. Therefore, the algorithm iterates through potential contact forces until the system of energy equations is satisfied. In other words, this algorithm finds the portion of the energy used by ice-crushing and the portion used due to structural deformation, for a specified contact force and compares the sum of those energies to the total kinetic energy of the system. Figure 3.1 shows the development of the energy distribution of the ice and structure interaction until the total kinetic energy is reached.

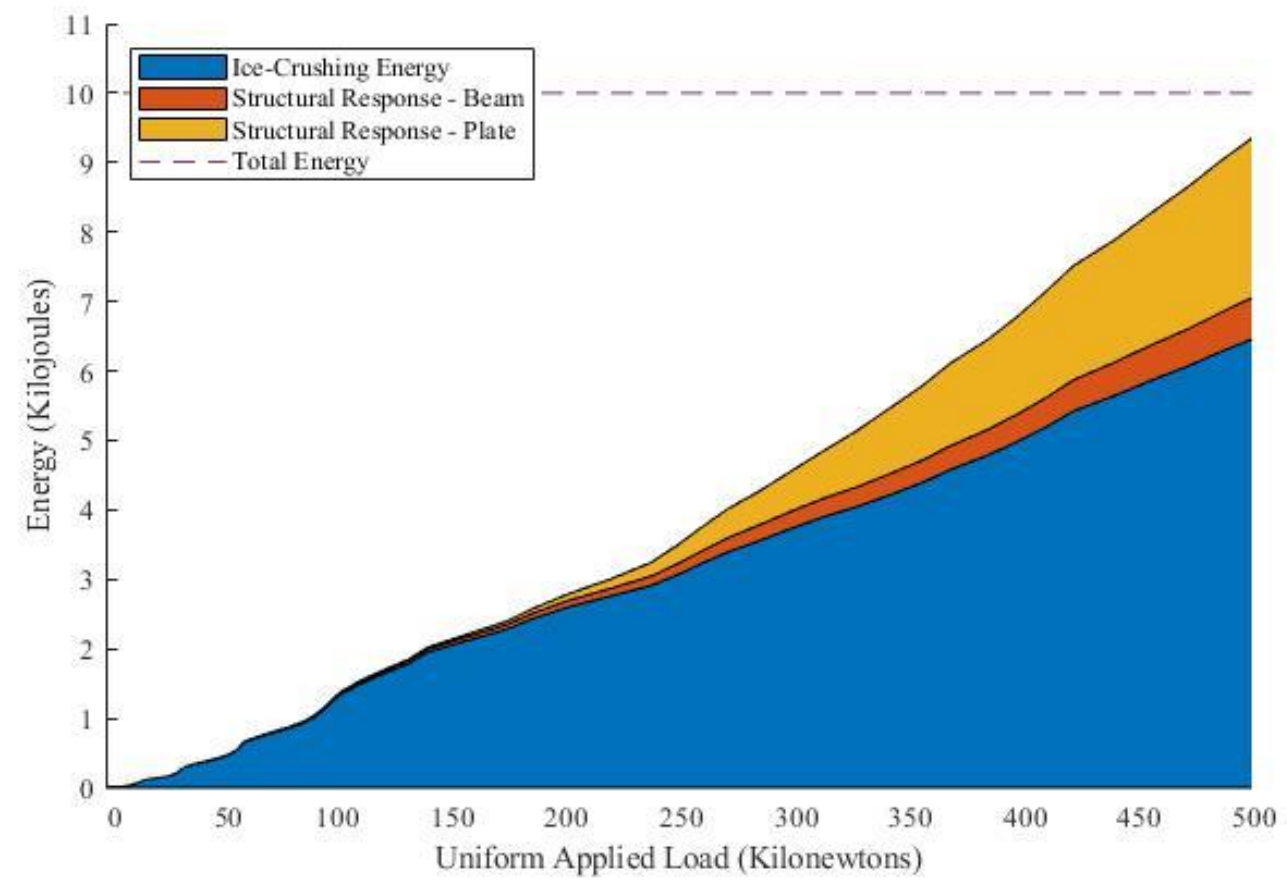

Figure 3.1 Ice and Structural Energy Distribution 
The algorithm repeats this assessment and iterates through possible contact forces until the summed ice-crushing energy and structural response energy equals the total kinetic energy of the ship-ice interaction. Figure 3.2 shows the algorithm mapped out in broad terms, to help visualize the process. The rest of this chapter explains the specific components of the algorithm, and an example MATLAB script of the complete algorithm is included as Appendix A.

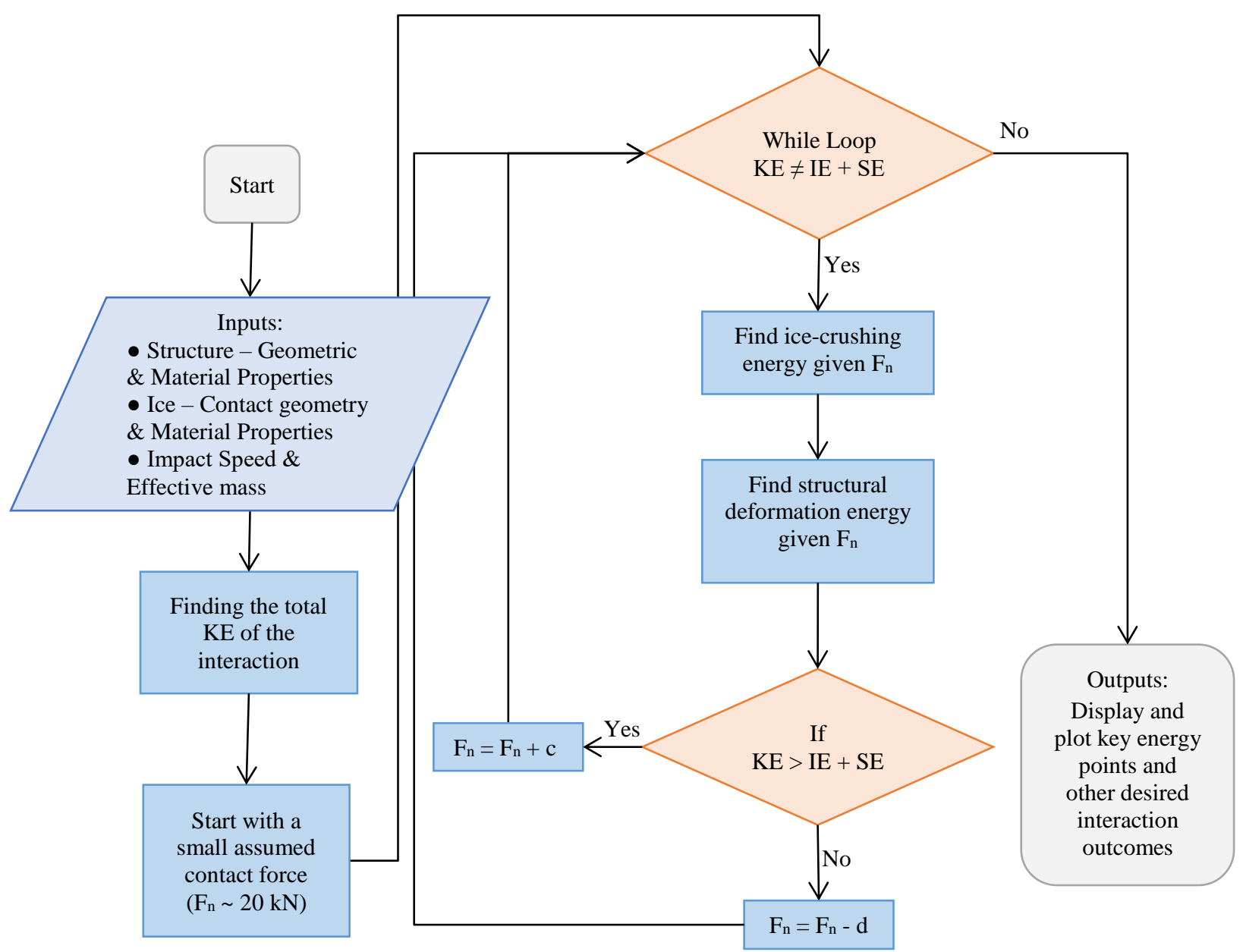

Figure 3.2 Algorithm General Approach Flowchart 


\subsubsection{Inputs and Outputs}

Specific parameters needed for both the ice and ship components of the interaction can be found in Table 3.1. The potential applications of this algorithm include assessing or modeling a specific ship interacting within an ice field. Therefore, easy access to the ship's structural parameters is assumed. For the ship inputs, the mass, basic ship parameters, velocity, and standard ship structure specifications are required.

Table 3.1 Input Variables

\begin{tabular}{|c|c|c|c|}
\hline \multicolumn{2}{|l|}{ Ship } & \multicolumn{2}{|l|}{ Ice } \\
\hline Velocity & $(\mathrm{m} / \mathrm{s})$ & Velocity & $(\mathrm{m} / \mathrm{s})$ \\
\hline Mass & $(\mathrm{kg})$ & Ice Density & $\left(\mathrm{kg} / \mathrm{m}^{3}\right)$ \\
\hline Draft & (m) & \multirow{2}{*}{$\begin{array}{l}\text { Ice Length/ Width (assuming a } \\
\text { square geometry) }\end{array}$} & \\
\hline Beam & (m) & & (m) \\
\hline Length Overall & (m) & Ice Thickness & (m) \\
\hline Height to Main Deck & (m) & Ice Strength & $(\mathrm{Pa})$ \\
\hline Midship Coefficient & $(-)$ & Ice-Crushing Exponent & $(-)$ \\
\hline Waterplane Coefficient & $(-)$ & Wedge Angle & (rad) \\
\hline Block Coefficient & $(-)$ & \multirow{2}{*}{$\begin{array}{l}\text { Impact Location (Coordinates } \\
\text { from Center of Mass) }\end{array}$} & \multirow[b]{2}{*}{ (m) } \\
\hline Waterline Angle & (rad) & & \\
\hline Frame Angle & $(\mathrm{rad})$ & & \\
\hline Normal Frame Angle & (rad) & & \\
\hline Shear Angle & $(\mathrm{rad})$ & & \\
\hline $\begin{array}{l}\text { Impact Location (Coordinates } \\
\text { from Center of Mass) }\end{array}$ & (m) & & \\
\hline \multicolumn{2}{|l|}{ Material Properties } & & \\
\hline Density & $\left(\mathrm{kg} / \mathrm{m}^{3}\right)$ & & \\
\hline Young's Modulus & $(\mathrm{Pa})$ & & \\
\hline Yield Strength & $(\mathrm{Pa})$ & & \\
\hline \multicolumn{2}{|l|}{ Beam } & & \\
\hline Length & (m) & & \\
\hline Cross-Section Dimensions & (m) & & \\
\hline \multicolumn{2}{|l|}{ Plate } & & \\
\hline Thickness & (m) & & \\
\hline Length & (m) & & \\
\hline Height & (m) & & \\
\hline Fixed Patch Load Length & (m) & & \\
\hline
\end{tabular}


When modelling a ship-ice interaction, the ice properties and geometry, in reality, are more likely to vary, especially compared to the ship structure. The algorithm has the potential to vary the ice parameters and contact geometry or allow them to be set by a designer. For the example algorithm, in Appendix A, fixed ice parameters and contact geometries are set to simplify the assembly of the algorithm. Nonetheless, the algorithm requires the ice velocity, mass, geometry, and standard ice properties, like ice strength, thickness, and density.

As the algorithm is arranged to solve for the collision energies and contact force, other desired outputs can also be determined once the energy distribution is known. For instance, the algorithm can also be set to solve for the ice and structural displacement to allow for force-displacement plots of the structure and ice response during the collision.

\subsubsection{Assumptions}

The ice-structure interaction response algorithm is created to model the ship's response within the bounds of the operational limits of the ship's structure following the IACS Polar rules standards [1]. Therefore, the IACS Polar rules are the basis for the assumptions and theories used in the algorithm. The main assumptions and methodologies that the algorithm follows regarding the loading event from the IACS Polar rules are the design scenario of a glancing impact and a Popov-based loading event [2]. The ice is assumed to produce a maximum contact force due to crushing, but unlike the IACS UR, the limiting force of the ice in flexure due to bending is not considered in this algorithm [19]. The plate and framing structural components experience a uniformly distributed rectangular patch load from the ice contact and follow the IACS Polar rules allowable limit state of 3-hinge collapse due to 
bending [43]. The algorithm does not include a shear criterion, like the one adopted in the IACS Polar rules framing standards.

The algorithm uses Popov's collision model to find the kinetic energy created during the collision [2]. Popov's collision model assumes that the interaction is instantaneous and that the ice is normal to the hull. In addition, friction, buoyancy forces, and dampening from the water are ignored during the collision [4]. Likewise, this algorithm assumes that all the initial collision energy and initial kinetic energy goes into either ice-crushing or structural deflection. Therefore, this model assumes that the instantaneous interaction ends with a normal kinetic energy of zero.

The ice-crushing model assumes a constant uniform area, with a slight area increase or decrease with the change in contact force, following the pressure-area relationship [3]. Further, the ice model ignores any variation in spatial pressure and high-pressure zones caused by the ice-crushing process, therefore assuming the ice contact produces an average uniform pressure on the ship structure. In addition to assuming the ice only fails due to crushing, by assuming the ice interaction is instantaneous the possibility of the ice rotating or rafting can be ignored. Otherwise, the ice-crushing model follows similar assumptions as Popov as the implemented ice collision model was developed in conjunction with the Popov collision model [3].

To solve for the structural response energy, first, the grillage structure is separated into simplified plate and beam components. Even though the system is anticipated to be nonlinear, diminishing the applicability of superposition, it was still decided to model the structural components separately to simplify the calculations of the structural response. The 
expected errors with this assumption are assessed later during benchmarking. In addition, the structural model assumes a localized response, ignoring any exterior ship motion not normal to the collision surface, since this model is only considering minimal permanent set during any one collision. Finally, following the assumptions of the ice model, the ice load is applied to the structural components as a rectangular patch load with a uniform distribution.

\subsubsection{Termination Condition}

With the IACS Polar rules as the basis for the assumptions and theories used in the algorithm, this algorithm is set up to model anything in the elastic or plastic material response region before the center hinge collapse of any of the structural components. Therefore, once the termination condition, the upper limit of the response range of the algorithm, of 3-hinge collapse is met, the algorithm ceases computation and does not compute the interaction response past that point. The centre or 3-hinge collapse limit state allows some plastic deformation before any substantial deterioration of the structural capacity of the ship occurs [50]. Even with the allowance of some plastic deformation, the 3-hinge collapse limit state is still considered a conservative model, since in reality there are a number of additional collapse mechanisms, like shear and axial tension, that are not being considered and contributing to the structural response.

The 3-hinge collapse in this algorithm assumes the plate or beam first forms theoretical plastic hinges at the edges of the element, followed by a third plastic hinge at the center of the structural component. Both beams and plates initially yield at the edges, indicating a transition from the purely elastic material response. Once the edges of these structural 
components have yielded through the entire cross-section, to simplify the response, the yielded regions are treated as plastic hinge moments. Simultaneously, the rest of the material is still modelled elastically, thus allowing a simplified initial plastic response. Therefore, once the center of the structural component reaches yield within the simplified initial plastic response and the center hinge forms, this creates a third hinge at the center of the element and indicates a 3-hinge collapse [45, 49].

This model separates the degrees of damage the structural response could experience into three basic categories, for both efficiently communicating the damage due to the interaction to the user and helping organize the algorithm into discrete modules. When the structural response is elastic, the behaviour is considered safe. When the structure deforms plastically up to the limit state of 3-hinge collapse, the structural response is within the cautionary zone, but still within the design limit of the structure. Once the structural response surpasses the limit state, the code terminates and deems the interaction past the operational design limit of the structure.

\subsection{Initial Kinetic Energy of the Collision}

To distribute the energy between the ice and the structure, first, the total kinetic energy of the system must be determined. To establish the kinetic energy of the interaction, Popov's collision method is implemented [2]. Popov's ship-ice interaction model is appropriate, as it simplifies a complex six degrees of freedom problem into a single degree of freedom. The main assumptions of Popov's method, as discussed in the previous section, are that all the energy is absorbed during the interaction resulting in a final kinetic energy of zero, that the collision occurs normal to the ship hull, and that the interaction is instantaneous. In 
addition, Popov's method neglects the forces produced by friction, damping and buoyancy effects, to help make the equations solvable, even though neglecting these factors increases the error produced by the model [4].

The kinetic energy of the ship-ice interaction (Eq. 2) requires the reduced mass of the system (Eq. 3) and the relative normal velocity $\left(V_{n}\right)$ of the ship and ice. To determine the reduced mass of the system (Eq. 3) first requires the reduced masses of the individual colliding bodies $\left(M_{e_{\text {ship }}} \& M_{e_{i c e}}\right)$. The effective or reduced mass of the system and individual bodies essentially summarizes the inertial effects experienced and produced by the individual bodies involved in the collision. The effective mass of the ship and ice takes into account the location of the impact relative to the object's center of mass considering effects like the radii of gyration and the moment arms. With this approach of a singular effective mass $\left(M_{e}\right)$, the inertial effects produced in the six possible degrees of freedom are summarized into a single term.

$$
\begin{gathered}
K E=1 / 2 M_{e_{s y s}} V_{n}^{2} \\
M_{e_{s y s}}=\frac{1}{\frac{1}{M_{e_{\text {ship }}}}+\frac{1}{M_{e_{i c e}}}}
\end{gathered}
$$

Popov [2] developed the reduced mass approach with a combination of theoretical and experimental equations. The reduced mass of the ship or ice (Eq. 4) represents how a point on the object will accelerate subjected to a normal force, and modelled with the object's mass $(M)$ and effective mass coefficient $\left(C_{o}\right)$. The effective mass coefficient (Eq. 5) is dependent on the following inertial properties: the directional cosines $(l, m, n)$, moment 
arms $(\lambda, \mu, \eta)$, squared radii of gyration $\left(r x^{2}, r y^{2}, r z^{2}\right)$, and the added mass terms $\left(A M_{x}, A M_{y}\right.$, $\left.A M_{z}, A M_{\text {roll }}, A M_{\text {pit }}, A M_{\text {yaw }}\right)$. Popov's original reduced mass equations assumed a circular ice floe, but the example script in Appendix A assumed a square ice floe with add mass terms adapted by Dolny [51]. More details about the terms in the effective mass coefficient equation and Popov's collision model can be found in Appendix B.

$$
\begin{gathered}
M_{e}=\frac{M}{C_{o}} \\
C_{o}=\frac{l^{2}}{1+A M_{x}}+\frac{m^{2}}{1+A M_{y}}+\frac{n^{2}}{1+A M_{z}}+\frac{\lambda^{2}}{r x^{2}\left(1+A M_{\text {roll }}\right)}+\frac{\mu^{2}}{r y^{2}\left(1+A M_{\text {pit }}\right)}+\frac{\eta^{2}}{r z^{2}\left(1+A M_{\text {yaw }}\right)}
\end{gathered}
$$

\subsection{Ice-Crushing Energy}

Daley's energy based ice collision force models [3] are used to determine the ice-crushing energy. Generally, Daley's models find the normal force of the impact, through a forceindentation relationship, knowing the kinetic energy of the system, typically found with Popov [2]. The models use the pressure-area relationship to determine the contact force and integrate the force to determine the ice indentation energy.

First, a generic pressure-area relationship is found (Eq. 6) dependent on the nominal pressure at $1 \mathrm{~m}^{2}\left(P_{o}\right)$, the contact area $\left(A_{c}\right)$, and the ice-crushing exponent $(e x)$. From there the pressure-area relationship is used to determine the force $(F)(\mathrm{Eq} .7)$.

$$
\begin{aligned}
& P_{\text {ave }}=P_{o} A_{c}{ }^{e x} \\
& \text { Eq. } 6 \\
& F=P_{a v e} A_{c}=P_{o} A_{c}^{1+e x}
\end{aligned}
$$

Now, an appropriate contact area needs to be determined to find the contact force $\left(F_{n}\right)$ between the structure and the ice during the interaction. To do this, the normal contact geometry $\left(A_{n}\right)$ is assumed to be a function $\left(f_{A}\right)$ dependent on the contact area and the normal 
indentation of the ice $\left(\zeta_{n}\right)$, Eq. 8. Similarly, the contact force $\left(F_{n}\right)$ (Eq. 9) is assumed to be a function $\left(f_{F}\right)$ relating force to the normal indentation $\left(\zeta_{n}\right)$. The force function (Eq. 10) assumes a similar form as Eq. 7, but in this case, the contact area is replaced by the normal contact geometry (Eq. 8).

$$
\begin{aligned}
& A_{n}=f_{A}\left(\zeta_{n}\right) \\
& \text { Eq. } 8 \\
& F_{n}=f_{F}\left(\zeta_{n}\right) \\
& \text { Eq. } 9 \\
& f_{F}=P_{o}\left(f_{A}\left(\zeta_{n}\right)\right)^{1+e x}
\end{aligned}
$$

With a relationship now determined for the contact force based on the normal contact geometry, the force is integrated relative to the normal ice indentation to solve for the ice indentation energy (IE), also known as ice-crushing energy (Eq. 11).

$$
I E=\int F_{n} d \zeta_{n}=\int P_{o}\left(f_{A}\left(\zeta_{n}\right)\right)^{1+e x} d \zeta_{n}
$$

Next, the normal contact geometry is determined depending on the shape of the ice in the interaction. The current model assumes a single geometry of a wedge normal to the hull (Figure 3.3), but Daley [3] derived many different contact geometries, allowing for various types of interactions or the potential for an algorithm with varying contact geometries depending on the desired use of the algorithm.

With the contact geometry chosen, the projected vertical, horizontal, and normal areas can be determined. To solve for the contact force $\left(F_{n}\right)$, only the normal contact area $\left(A_{n}\right)$ is required. The normal contact area (Eq. 12) is found dependent on the normal frame angle $\left(\beta^{\prime}\right)$ of the ship hull, the ice-wedge angle $(\varphi)$, and the normal ice indentation $\left(\zeta_{n}\right)$. From there the normal projected contact area is substituted into Eq. 9 to solve for the contact 
force (Eq. 13). Finally, the contact force is substituted into Eq. 11 and integrated to solve for the ice-crushing energy (IE) (Eq. 14).
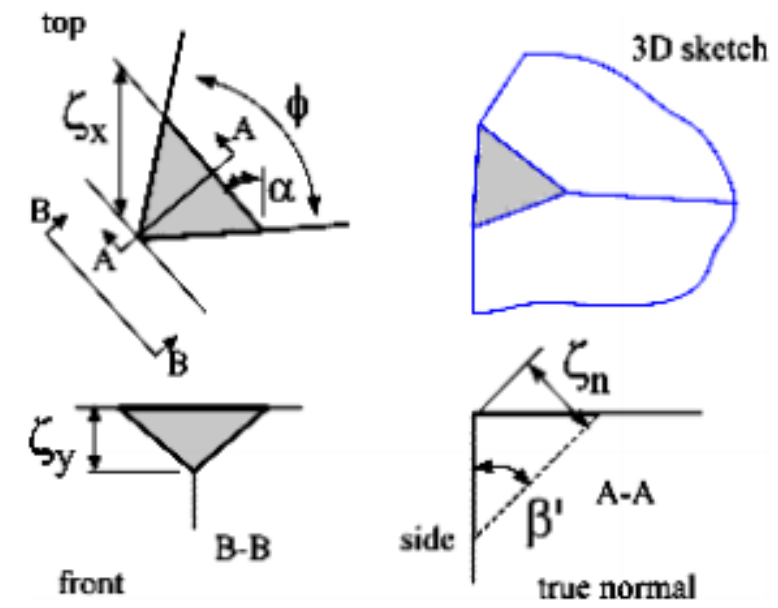

Figure 3.3 Wedge Normal to the Hull Contact Geometry [3]

$$
\begin{gathered}
A_{n}=\frac{\zeta_{n}^{2} \tan \left({ }^{\varphi} / 2\right)}{\sin \left(\beta^{\prime}\right) \cos ^{2}\left(\beta^{\prime}\right)} \\
F_{n}=P_{o}\left(\frac{\tan \left({ }^{\varphi} / 2\right)}{\sin \left(\beta^{\prime}\right) \cos ^{2}\left(\beta^{\prime}\right)}\right)^{1+e x} \zeta_{n}^{2+2 e x} \\
I E=\frac{P_{o}}{3+2 e x}\left(\frac{\tan \left({ }^{\varphi} / 2\right)}{\sin \left(\beta^{\prime}\right) \cos ^{2}\left(\beta^{\prime}\right)}\right)^{1+e x} \zeta_{n}^{3+2 e x}
\end{gathered}
$$

To simplify the ice-crushing energy equation (Eq. 14) two contact geometry specific terms ( $f_{a}$ and $f_{x}$ ) were established. These contact geometry terms can vary depending on the type of ice edge geometry the ice is modelled with and are dependent on the ice-crushing constant $\left(e_{x}\right)$, normal frame angle $\left(\beta^{\prime}\right)$, and ice-wedge angle $(\varphi)$. Assuming a general wedge normal to the hull contact geometry gives the contact geometry terms found as Eq. 15 and 
Eq. 16. From there, Eq. 17 is determined by substituting in the contact geometry specific terms, and by solving the contact force (Eq. 13) for the normal ice indentation and substituting that into Eq. 14, finally creating an ice-crushing energy (IE) dependent on the contact force $\left(F_{n}\right)$.

$$
\begin{gathered}
f_{x}=3+2 e_{x} \\
f_{a}=\left(\frac{\tan (\varphi / 2)}{\sin \left(\beta^{\prime}\right) \cos ^{2}\left(\beta^{\prime}\right)}\right)^{1+e_{x}} \\
I E=\frac{P_{o} f_{a}}{f_{x}}\left(\frac{F_{n}}{P_{o} f_{a}}\right)^{f x / f x-1}
\end{gathered}
$$

\subsubsection{Rectangular Patch Load Dimensions}

A rectangular patch load, with a similar aspect ratio to the original contact geometry, can be arranged with the geometric parameters of the ice's contact geometry, Figure 3.4. This approach determines the rectangular patch load dimensions based on the ice indentation and contact geometries [51]. Comparatively, the IACS Polar rules establish the rectangular patch load based on the hull angles, ship displacement, and a set aspect ratio for non-bow patch loads [1]. By determining the patch load based on the ice force-indentation relationship, the patch load dimensions vary depending on the amount of force produced during the interaction, rather than the fixed patch load dimensions of the IACS Polar rules.
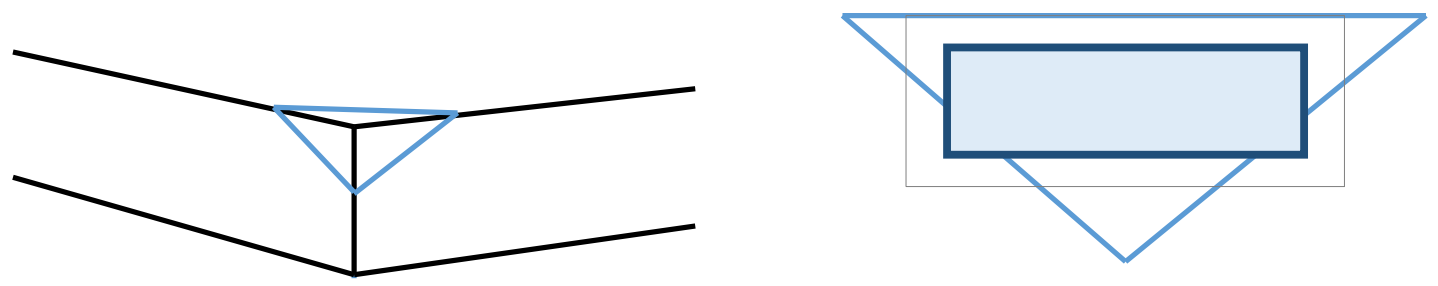

Figure 3.4 Wedge Normal to the Hull Contact Geometry Conversion to a Rectangular Patch Load 
To convert the triangular contact surface between the ice and ship structure that forms for a wedge normal to the hull contact geometry, the algorithm adapts the approach presented by Dolny [51], which follows a similar approach to the IACS Polar rules but is dependent on the normal indentation of the ice instead of the displacement of the ship. The triangular width (Eq. 18) is found dependent on the normal indentation of the ice $\left(\zeta_{n}\right)$, the ice-wedge angle $(\varphi)$, and the normal frame angle $\left(\beta^{\prime}\right)$. Similarly, the aspect ratio (Eq. 19) is found with the wedge angle of the ice, and the normal frame angle of the ship structure [51]. The triangular width is then used to find the nominal width (Eq. 20) for a rectangle. With the nominal rectangular patch load width and the aspect ratio, a reduced patch load height and width (Eq. 21 and Eq. 22) are found. A reduced patch load is implemented to model a more concentrated load at the centre of the original patch load and reduce the area of the applied load, which accounts for some spalling at the edges [51].

$$
\begin{gathered}
W_{z}=\frac{2 \zeta_{n} \tan (\varphi / 2)}{\cos \left(\beta^{\prime}\right)} \\
A R=2 \tan (\varphi / 2) \sin \left(\beta^{\prime}\right) \\
W_{n o m}=W_{z} / \sqrt{2} \\
w_{p l}=0.7 \cdot W_{n o m} \\
h_{p l}={ }^{w_{p l}} / A R
\end{gathered}
$$

From there, the rectangular dimensions can be applied to the structural components as patch loads. The width $\left(w_{p l}\right)$ is applied as the beam's patch load length, and the height $\left(h_{p l}\right)$ is used as the patch load height on the plate. 


\subsection{Structural Energy}

There are no pre-existing structural models that would fit in the framework of this algorithm and determine the energy that a grillage structure experiences during a collision. Therefore, to determine the structural response energy of a grillage, assuming a localized collision, the energy response is calculated for the individual structural components and superimposed. Similar to the structural analyses of the IACS Polar rules, the structure model separates the grillage structure into two types of structural elements, plates and beams, and so the algorithm requires both a beam and plate response energy model.

Both the beam and plate response energy models are divided into two parts, an elastic and plastic response, to allow good model prediction capabilities. The beam model is split into two to allow for a shift in the approach of how the fixed boundary condition is modelled between the two material responses. As the plate response is described using a meta-model, the model is divided to improve the estimation of the generated meta-model and to account for the slight shift between the shape of the elastic and plastic response of the plate. In both cases, the shift between the elastic and plastic models occurs once the edges of the component experience full through-thickness yield.

\subsubsection{Beam Model}

Classical beam theory is used to find the deformation energy of the beam. This model only considers the bending energy and ignores axial tension. A simple explicit FEA analysis is conducted to assess the validity of this assumption. Two $180 \mathrm{~mm}$ thick and $9 \mathrm{~mm}$ wide rectangular cross-section beam models are created using beam elements and an elastic perfectly plastic material model and the properties of structural steel: a density of 7850 
$\mathrm{kg} / \mathrm{m}^{3}$, a Young's modulus of $200 \mathrm{GPa}$, a Poisson's ratio of 0.3 , and a yield strength of 250 MPa. A $191.5 \mathrm{kN} / \mathrm{m}$ load is applied as a beam load across the full length of the $1.5 \mathrm{~m}$ long beam to model a uniform distributed load. In this case, a rectangular cross-sectioned beam is assessed since a rectangular cross-section is assumed and used to create the sample algorithm. Only the boundary conditions changed between the two models: one beam is fixed to induce a bending and axial response, while the other beam is fixed but is free-topull in and move in the longitudinal direction to produce a purely bending beam response.

As can be seen from Figure 3.5, the FEA analysis shows no significant increase in energy with the inclusion of the axial tension. Therefore, in this specific case of a simple rectangular beam, a fixed but free-to-slide boundary condition is implemented to induce a purely bending response.

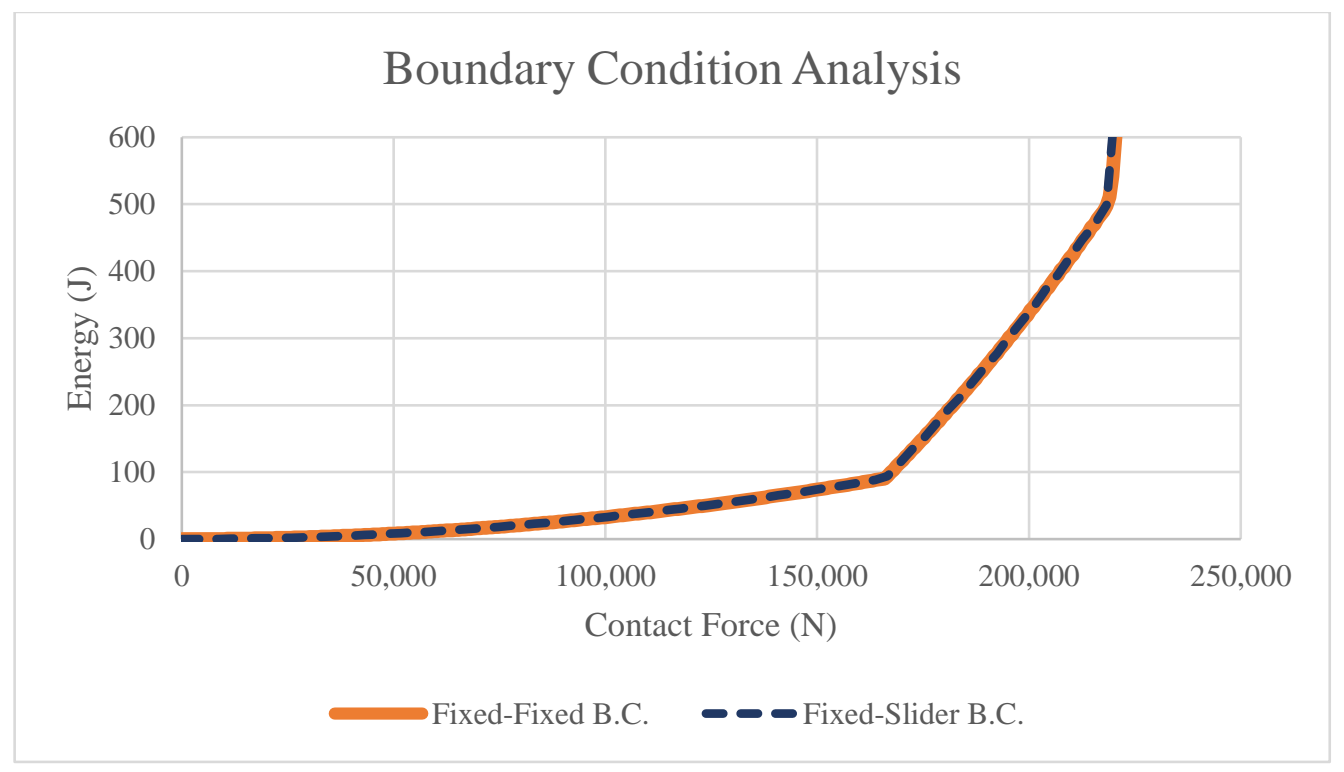

Figure 3.5 Beam Deformation Response Energy 
The elastic strain energy due to flexure is employed to find the beam bending energy. With this approach, the moment distribution across the length of the beam can be found for different boundary and loading conditions and substituted into the energy equation (Eq. 23). The strain energy $(U)$ is derived from the moment $(M(x))$ across the length of the beam $\left(L_{b}\right)$, Young's modulus $(E)$, and the moment of inertia $(I)[52]$.

$$
U=\int_{L_{b}} \frac{M^{2}(x)}{2 E I} d x
$$

In this case, the beam model assumes a uniformly distributed patch load centred on the beam with a variable patch length as the loading condition, to mimic the change in contact area dependent on the magnitude of the contact force.

\subsubsection{Elastic Model}

To find the deformation energy of the beam in the elastic material response region, first, the moment formula across the beam is established. As mentioned above, the beam has a fixed but free-to-pull in boundary condition, with a variable patch load length, centred on the beam (Figure 3.6). The moment equation (Eq. 24) is determined with classical beam theory and represented with the use of piecewise functions. A more detailed derivation of the beam equations is found in Appendix C.

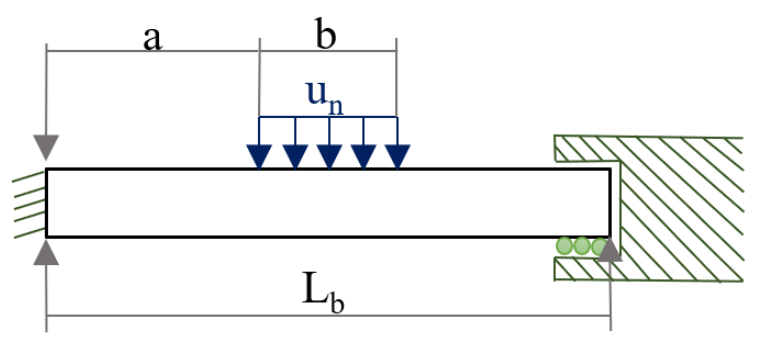

Figure 3.6 Elastic Beam Boundary and Loading Conditions 


$$
\begin{aligned}
& R_{A e}=\frac{u_{n}}{2 L_{b}^{3}}\left[\left(L_{b}-a-b\right)^{4}-\left(L_{b}-a\right)^{4}\right] \\
& +\frac{u_{n}}{L_{b}^{2}}\left[\left(L_{b}-a\right)^{3}-\left(L_{b}-a-b\right)^{3}\right] \\
& M_{A e}=\frac{u_{n}}{4 L_{b}^{2}}\left[\left(L_{b}-a-b\right)^{4}-\left(L_{b}-a\right)^{4}\right] \\
& +\frac{u_{n}}{3 L_{b}}\left[\left(L_{b}-a\right)^{3}-\left(L_{b}-a-b\right)^{3}\right] \\
& M_{e_{1}}(x)=-M_{A e}+R_{A e^{x}} \quad 0 \leq x \leq a \\
& M_{e_{2}}(x)=-M_{A e}+R_{A e} x-\frac{u_{n}}{2}(x-a)^{2} \quad a \leq x \leq a+b \\
& M_{e_{3}}(x)=-M_{A e}+R_{A e} x-u_{n} b(x-a-b / 2) \quad a+b \leq x \leq L_{b}
\end{aligned}
$$

The bending moment across the beam is found with the elastic reaction force $\left(R_{A e}\right)$ and elastic reaction moment $\left(M_{A e}\right)$ at the start of the beam, the uniform load $\left(u_{n}\right)$, the distance to the beginning of the applied load $(a)$, the length of the applied patch load $(b)$, and the full length of the beam $\left(L_{b}\right)$. From there, the moment equation (Eq. 24) is substituted into the energy equation (Eq. 23) to be integrated across the full length of the beam, to determine the elastic bending energy $\left(U_{e}\right)$ of the beam (Eq. 25). The integral is solved using MATLAB and then copied into the algorithm. The beam strain energy integration script and solution are found in Appendix D. 


$$
\begin{aligned}
U_{e}=\frac{1}{2 E I}\left[\int_{0}^{a}\right. & \left(-M_{A e}+R_{A e} x\right)^{2} d x \\
& +\int_{a}^{a+b}\left(-M_{A e}+R_{A e} x-\frac{u_{n}}{2}(x-a)^{2}\right)^{2} d x \\
& \left.+\int_{a+b}^{L_{b}}\left(-M_{A e}+R_{A e} x-u_{n} b(x-a-b / 2)\right)^{2} d x\right]
\end{aligned}
$$

\subsubsection{Plastic Model}

As the model is only modelling the initial portion of the plastic material response, the material response is still linear and is often considered the pseudo-elastic region [53]. As the majority of the beam is still responding elastically, elastic theory is still applied, but the boundary conditions are adapted to model the new material behaviour. Following an elastic perfectly plastic material model, the ends of the beam are replaced with plastic hinge moments, since the edges yield first [48]. Thus, the adapted boundary condition for the beam is simply supported with imposed plastic moments at both ends, Figure 3.7.

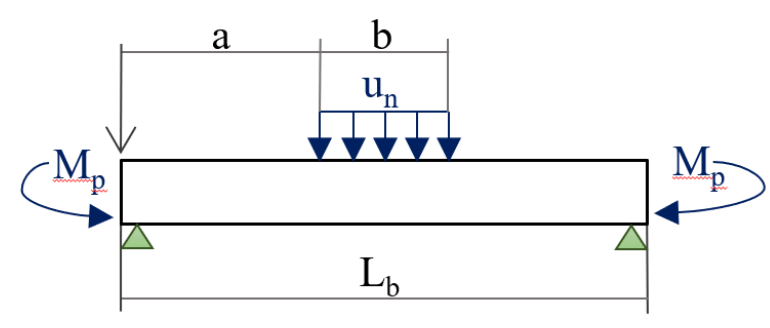

Figure 3.7 Plastic Beam Boundary and Loading Conditions

With the modified boundary condition and the centred patch load loading condition (Figure 3.7), the moment across the beam (Eq. 26) is derived, with details shown in Appendix C. The moment $(M(x))$ is dependent on the plastic moment $\left(M_{p}\right)$, the plastic reaction force 
$\left(R_{A p}\right)$ at the start of the beam, the uniform load $\left(u_{n}\right)$, distance to the start of the applied load (a), length of the patch load $(b)$, and the full length of the beam $\left(L_{b}\right)$.

$$
\begin{aligned}
& R_{A p}=\frac{u_{n} b}{L_{b}}\left(L_{b}-a-b / 2\right) \\
& M_{p_{1}}(x)=-M_{P}+R_{A p} x \\
& =-M_{P}+u_{n} b x-\frac{u_{n} a b x}{L_{b}}-\frac{u_{n} b^{2} x}{2 L_{b}} \\
& M_{p_{2}}(x)=-M_{P}+R_{A p} x-\frac{u_{n}}{2}(x-a)^{2} \\
& =-M_{P}+u_{n} b x-\frac{u_{n} a b x}{L_{b}}-\frac{u_{n} b^{2} x}{2 L_{b}}-\frac{u_{n} x^{2}}{2} \quad a \leq x \leq a+b \\
& +u_{n} a x-\frac{u_{n} a^{2}}{2} \\
& M_{p_{3}}(x)=-M_{P}+R_{A p} x-\frac{u_{n}}{2}(x-a)^{2} \\
& +\frac{u_{n}}{2}(x-(a+b))^{2} \\
& a+b \leq x \leq L_{b} \\
& =-M_{P}-\frac{u_{n} a b x}{L_{b}}-\frac{u_{n} b^{2} x}{2 L_{b}}+u_{n} a b-\frac{u_{n} b^{2}}{2} \\
& 0 \leq x \leq a
\end{aligned}
$$

The plastic moment is determined with the yield stress of the material $\left(\sigma_{y}\right)$ and plastic section modulus $(Z)$ of the beam's cross-section (Eq. 27). In the case of a rectangular crosssection of the beam, the plastic modulus (Eq. 28) consists of the cross-section thickness $(t)$ or distance to the neutral axis $(y)$, and width $(w)$. The plastic moment also assumes an elastic perfectly plastic material model, which follows the assumptions of the plastic hinge collapse model. 


$$
\begin{gathered}
M_{P}=\sigma_{y} Z \\
Z=\frac{t^{2} w}{4}=y^{2} w
\end{gathered}
$$

From there, the moment (Eq. 26) is substituted into the strain energy equation (Eq. 23) and integrated across the full length of the beam. Similar to the elastic response, the beam strain energy (Eq. 29) is integrated through MATLAB; the solution and script are found in Appendix D.

$$
\begin{aligned}
U_{p}=\frac{1}{2 E I}\left[\int_{0}^{a}(\right. & \left.-M_{P}+u_{n} b x-\frac{u_{n} a b x}{L_{b}}-\frac{u_{n} b^{2} x}{2 L_{b}}\right)^{2} d x \\
& +\int_{a}^{a+b}\left(-M_{P}+u_{n} b x-\frac{u_{n} a b x}{L_{b}}-\frac{u_{n} b^{2} x}{2 L_{b}}-\frac{u_{n} x^{2}}{2}+u_{n} a x\right. \\
& \left.-\frac{u_{n} a^{2}}{2}\right)^{2} d x \\
& \left.+\int_{a+b}^{L_{b}}\left(-M_{P}-\frac{u_{n} a b x}{L_{b}}-\frac{u_{n} b^{2} x}{2 L_{b}}+u_{n} a b-\frac{u_{n} b^{2}}{2}\right)^{2} d x\right]
\end{aligned}
$$

In addition, since the ends of the beam are now modelled as plastic hinge moments, work is also consumed through the rotation of the plastic hinges at the ends of the beam, which is not accounted for by the strain energy (Eq. 29). Therefore, the plastic response energy of the beam includes both the rotation work at the ends of the beam and the bending energy of flexure of the rest of the beam. The rotational work $\left(W_{R}\right)$ at the ends of the beam is dependent on the moment and angle of rotation in radians $(\theta)$, Eq. 30. In this case, the moment at the ends of the beam is the plastic moment $\left(M_{P}\right)$. 


$$
W_{R}=M_{P} \Delta \theta
$$

To determine the change in rotation of both ends of the beam, the beam slope equation at $x$ $=0$ and $x=L_{b}$ is derived. Applying classical beam theory with the already derived plastic response moment equation, finds the slope at the ends of the beam as Eq. 31. A more detailed derivation of the beam slope equation, $\theta(x)$, is shown in Appendix $\mathrm{C}$ with the beam moment derivations.

$$
\begin{array}{r}
\theta\left(0 \& L_{b}\right)=\frac{1}{E I L_{b}}\left[\frac{-M_{P} L_{b}^{2}}{2}+\frac{u_{n} b L_{b}^{2}}{6}\left(L_{b}-a-\frac{b}{2}\right)\right. \\
\left.+\frac{u_{n}}{24}\left(\left(L_{b}-a-b\right)^{4}-\left(L_{b}-a\right)^{4}\right)\right]
\end{array}
$$

As the elastic beam response assumes that the beam is fixed at both ends, the elastic response assumes a slope of zero at the ends of the beam during the whole response up to the transition to the plastic response. Therefore, the plastic change in angle of the hinge assumes an initial angle of zero. The full plastic response energy of the beam is shown as Eq. 32, where the energy is dependent on the flexure energy $\left(U_{p}\right)$, and the plastic hinge rotation work $\left(W_{R}\right)$ at both ends of the beam.

$$
\begin{aligned}
S E_{b_{p}}= & U_{p}+2 W_{R} \\
= & U_{p}+2 M_{P} \Delta \theta\left(0 \& L_{b}\right) \\
= & U_{p}+\frac{2 M_{P}}{E I L_{b}}\left[\frac{-M_{P} L_{b}^{2}}{2}+\frac{u_{n} b L_{b}^{2}}{6}\left(L_{b}-a-\frac{b}{2}\right)\right. \\
& \left.+\frac{u_{n}}{24}\left(\left(L_{b}-a-b\right)^{4}-\left(L_{b}-a\right)^{4}\right)\right]
\end{aligned}
$$




\subsubsection{Model Transition and Termination}

To indicate when the model transitions from an elastic to an initial plastic response, and terminates once center hinge collapse has occurred, moments are used. To determine when the beam yields at the ends and center of the beam, the moments at the ends and the center of the beam are compared to the plastic moment of the beam $\left(M_{p}\right)$. The moment equation derived to solve for the elastic energy equation (Eq. 24) is employed to solve for the bending moment at the ends of the beam $\left(x=0\right.$ or $\left.x=L_{b}\right)$ (Eq. 33), and happens to be the same as the elastic reaction moment equation $\left(M_{A e}\right.$, Eq. 24$)$. The transition from the elastic equation occurs once the moments at the ends of the beam exceed the plastic moment of the beam.

$$
M_{E}=\frac{u_{n}}{4 L_{b}^{2}}\left[\left(L_{b}-a-b\right)^{4}-\left(L_{b}-a\right)^{4}\right]+\frac{u_{n}}{3 L_{b}}\left[\left(L_{b}-a\right)^{3}-\left(L_{b}-a-b\right)^{3}\right]
$$

Similarly, the moment at the center of the beam (Eq. 34) is derived from the moment equation arranged to solve for the plastic response energy of the beam up to collapse (Eq. 26). Once the moment at the center of the beam $\left(x=L_{b} / 2\right)$ surpasses that of the plastic moment of the beam, this indicates center hinge collapse and the termination of the code.

$$
M_{c}=\frac{u_{n}}{4}\left[4 a^{2}-4 a L_{b}-2 a-b^{2}+2 b L_{b}-L_{b}^{2}\right]-M_{P}
$$

\subsubsection{Plate Model}

Various methods and analytical models were examined to create the plate model. None of the methods examined solved for the deformation energy of the plate or allowed a reasonable adaption to the method to solve for the energy. Most existing collision models 
for ships, either in ice or otherwise, focused on a singular design value [19, 37, 43]. Additionally, the design points were typically outside the elastic and plastic design range of this model [34, 39, 54]. One method examined was allowable permanent set [55], but this approach did not allow for modelling in the elastic range, and the model applicability terminated before the designated plate collapse. Also, the allowable permanent set was unable to be rearranged to solve for deformation energy. A few analytical models were promising but did not quite meet the criteria. Ratzlaff and Kennedy [56] developed an analytical plate model within the correct material response range, elastic and initial plastic response, but it could not be rearranged to solve for deformation energy. In addition, the primary assumption presented in Ratzlaff and Kennedy's model was a long plate with an infinite aspect ratio, limiting the adaptability of the model, even if the infinite aspect ratio model had a decent experimental agreement with reasonably low aspect ratio plates. Timoshenko [57] had the most promising analytical equations, as some of those equations actually solved for bending and membrane energy of the plate, but only a few specific cases had complete solutions. Trying to find the Timoshenko plate bending and membrane equations for other boundary or loading conditions was complex and out of the scope of this project.

As none of the examined analytical models met the criteria for this algorithm, a meta-model is created to represent the specific desired plate response. To do this the design of experiments (DoE) methodology is applied to extract data from FEA analyses. Then, with the data extracted from the computer experiments, a regression equation is created as a meta-model to model the internal energy of the plate examined during the experiments. 


\subsubsection{Design of Experiments - Uniform Design}

To simplify the inherently complex nature of plate bending and membrane response, a meta-model is created with a DoE approach to model the plate deformation energy. A uniform design DoE method [58] is used to create the meta-model. To collect the data and generate the meta-model, FEA is used to conduct the various loading experiments.

A uniform design is a space-filling design, which evenly distributes the design points across the response surface [58], which is desirable in cases such as this, where a deterministic computer model is used to conduct the experiments. This approach is preferred over other methods like response surface methodologies as it does not require duplicate runs, which are redundant with computer experiments [59]. For example, a center composite design (a response surface methodology) without duplicate runs loses its pure error terms, which reduces the modelling capability of the method from cubic to only quadratic, even though five levels are examined. In addition, with a uniform design, it is easy to increase the number of factor levels without outrageously increasing the required number of runs.

The plate meta-model is created with two input variables, contact force $(A)$ and patch load height $(B)$, and produces the output equation of the internal deformation energy of the plate. The uniform design is set with two five-level factors, and a 25 run experimental setup [60]. This design is selected as it has an adequate number of data points to allow for some pure error without requiring replication runs and provides up to a quartic model without significant aliased terms. Aliased terms occur when the full set of treatment combinations are not examined. Instead of all the possible factors and level combinations being examined, only a fraction of the terms are checked for significance. Only analyzing a 
fraction of the terms causes the higher-order terms to become aliased or grouped with specific lower-order terms, but these groups are represented by the lowest ordered term, leaving the significance of the higher-order terms unknown [59]. Depending on how many runs of the full treatment set are conducted, the factor and factorial interaction may be modelled together and prevent any meaningful conclusions from being drawn from the data.

The plate material response model is split into two different equations, the elastic and initial plastic response, to create models with good agreement and reasonably simple forms. This helps the models' predictability since the elastic and plastic material responses follow different forms. The elastic response energy is much more linear and grows gradually, while the plastic response is more exponential. However, as the model is split into two different response models a transition equation is required, in addition to a termination equation.

\subsubsection{Experimental FEA Models}

To create the meta-model, the experiments examine five levels for both factors. Therefore, only five FEA models are required with the various patch load heights. For the contact force, the FEA models only need to be examined up to the point where the center of the plate yields, the limit state of center hinge collapse. With the FEA models modelling the plate response up to yield at the center of the plate, the various responses throughout the interaction up to that point can be extracted for the desired contact force. The patch load is set to range from a relatively small height compared to the plate's height (for example, in this case, the smallest patch load height is roughly $1 / 15^{\text {th }}$ of the plate height) up to the full 
height of the plate. This is done to account for the variable contact areas, especially with lower forces, that the contact geometry between the plate and ice can produce.

The plate dimensions, for the model produced with this analysis, are determined by the distance between stiffeners for the height and the frame spacing determined by the plate width. For this case, a $600 \mathrm{~mm}$ wide by $300 \mathrm{~mm}$ tall plate is used to create the plate model and assumes the patch load heights range from $20 \mathrm{~mm}$ to the full plate height of $300 \mathrm{~mm}$ (Figure 3.8). The plate thickness is set for the desired region of the ship structure; in this case, the plate thickness is set to $12 \mathrm{~mm}$. Therefore, this plate model assumes fixed plate width, height, and thickness dimensions, with only the patch load height and contact force varying.
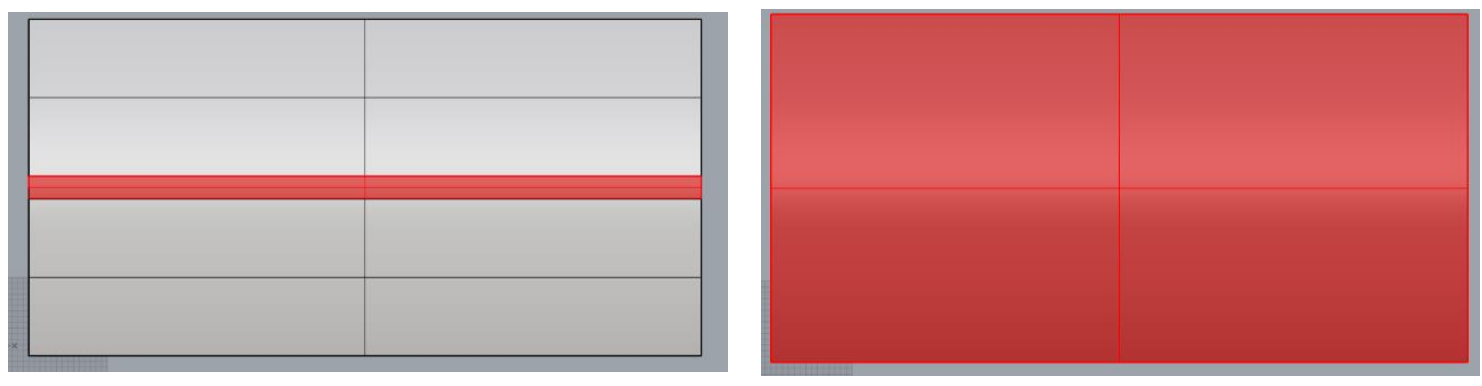

Figure 3.8 Patch Load Height Geometry, $2 \mathrm{~cm}$ and $30 \mathrm{~cm}$

The plate is created with shell elements with a shear factor of $5 / 6$ and 5 through-thickness integration points. The plate is set with fixed boundary conditions on all edges (Figure 3.9). Typically, analytical models assume a simply supported or free-to-pull in boundary condition to simplify the solution, which removes membrane effects from the problem, but for this model, a membrane response of the plate is desired. Therefore, the plate is set to experience a fixed boundary condition to induce both a bending and membrane response. 
For this model A36 structural steel is assumed, with a density of $7850 \mathrm{~kg} / \mathrm{m}^{3}$, a Young's Modulus of $200 \mathrm{GPa}$, a Poisson's ratio of 0.3 , and a yield strength of $250 \mathrm{MPa}$. A kinematic bi-linear material model is applied to model the initial plastic response of the plate. For the bi-linear model, an $E_{\text {tan }}$ of $1.5 \mathrm{GPa}$ is assumed, creating the material response shown in Figure 3.10.

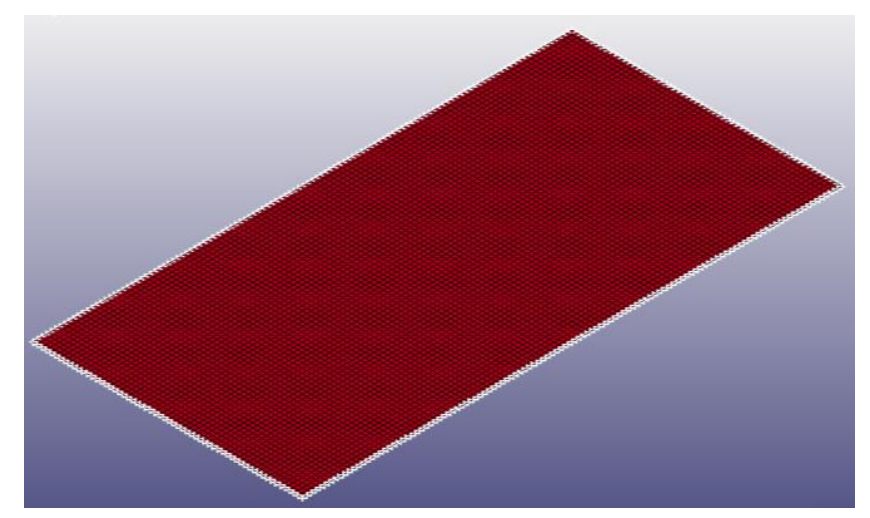

Figure 3.9 Boundary Condition

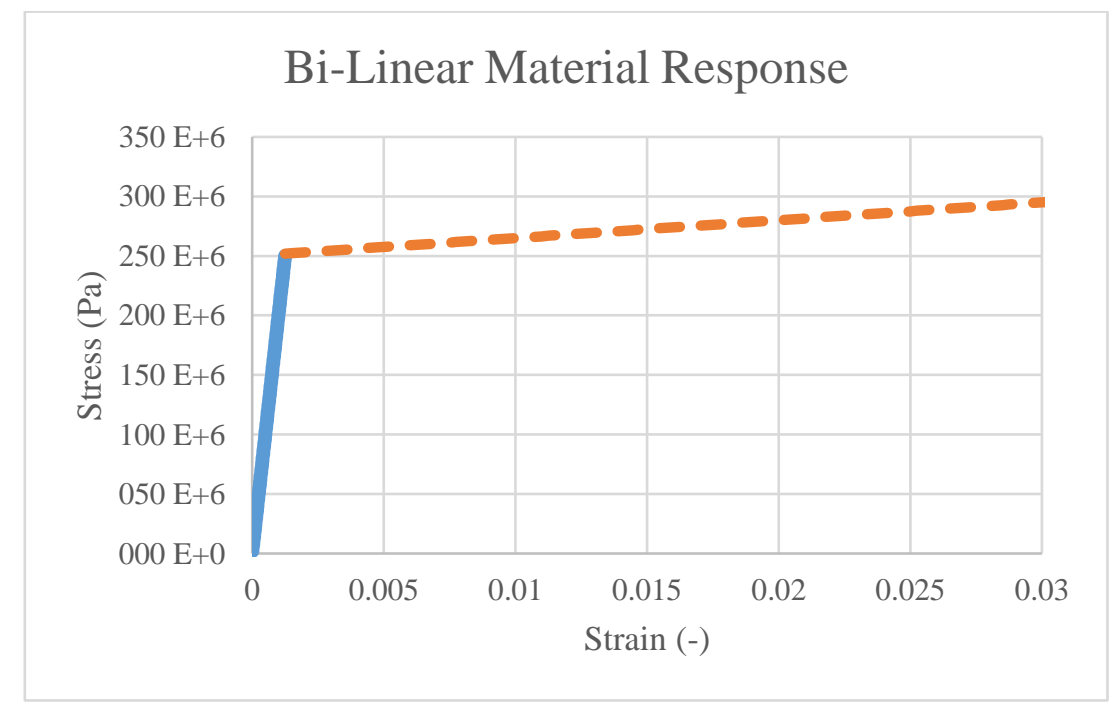

Figure 3.10 Elastic-Plastic Bi-linear Material Response

A distributed pressure is applied to a set of shell elements (Figure 3.11a), with a unit ramp function (Figure 3.11b). By applying the pressure with a unit ramp over one second 
gradually increases the load until the model reaches the full desired applied load of $535 \mathrm{kN}$ and removes any inertial response the plate may experience due to the applied load, thus creating a quasi-static loading event. Even though the load is applied in the FEA model as a pressure, the applied pressure is converted to a force when creating the meta-model. The default explicit solver is used to conduct the plate loading experiments.
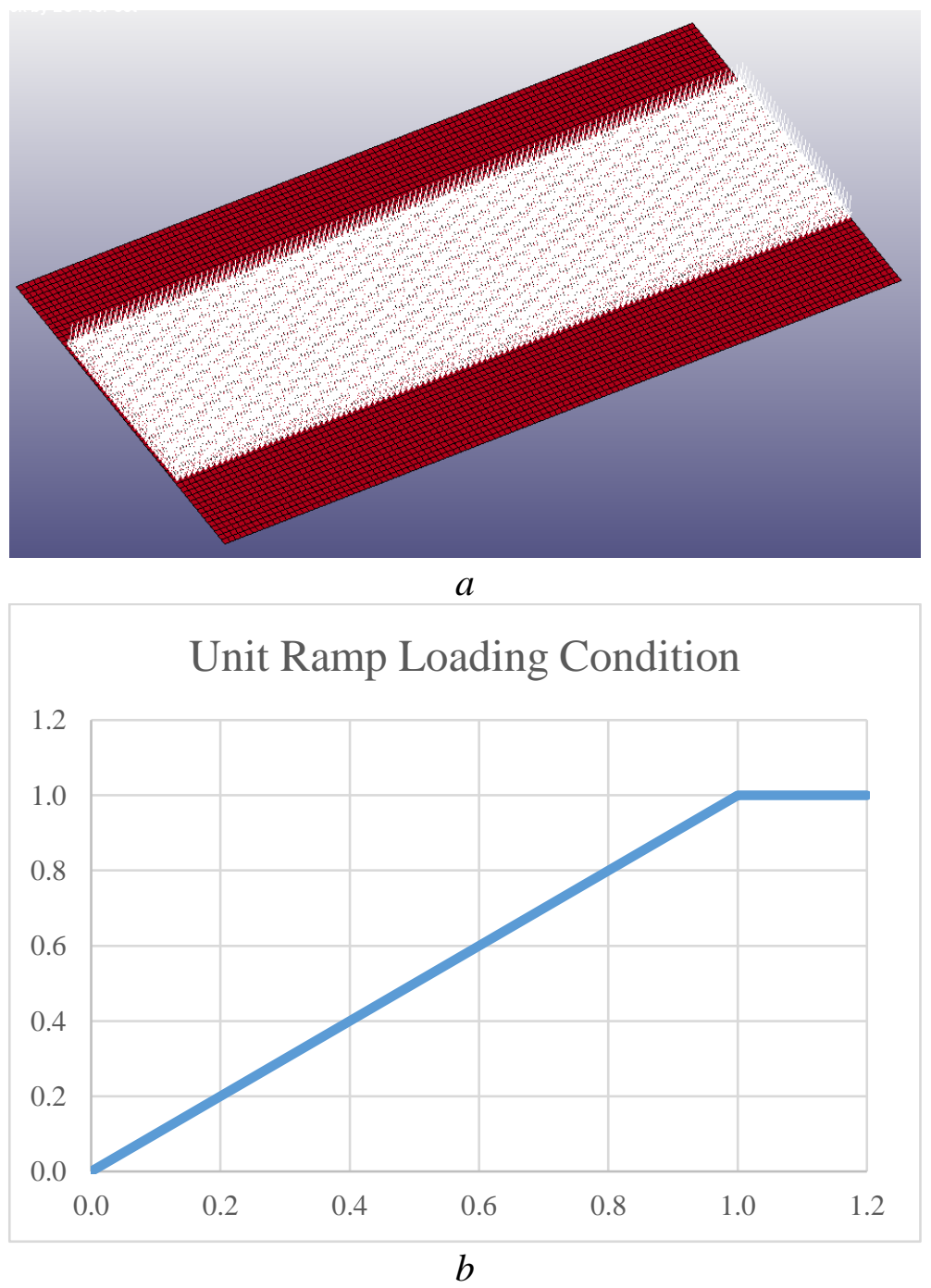

Figure 3.11 Patch Load Loading Condition

Before the final data is collected and implemented to create the meta-model, a mesh convergence analysis (Figure 3.12) is conducted for each of the five patch load heights used 
to create the model. Two mesh element sizes are chosen, $5 \mathrm{~mm}$ and $2 \mathrm{~mm}$ depending on the height of the patch load, to allow for a patch load mesh density of at least 4 elements per $\mathrm{cm}^{2}$. The smallest mesh element edge length of $2 \mathrm{~mm}$ allows for a height of 10 elements in the smallest patch load height of $2 \mathrm{~cm}$. A $2 \mathrm{~mm}$ mesh element length also provides the two $3.5 \mathrm{~cm}$ tall top and bottoms unloaded edges of the $23 \mathrm{~cm}$ patch load height plate, with a height of 18 elements.

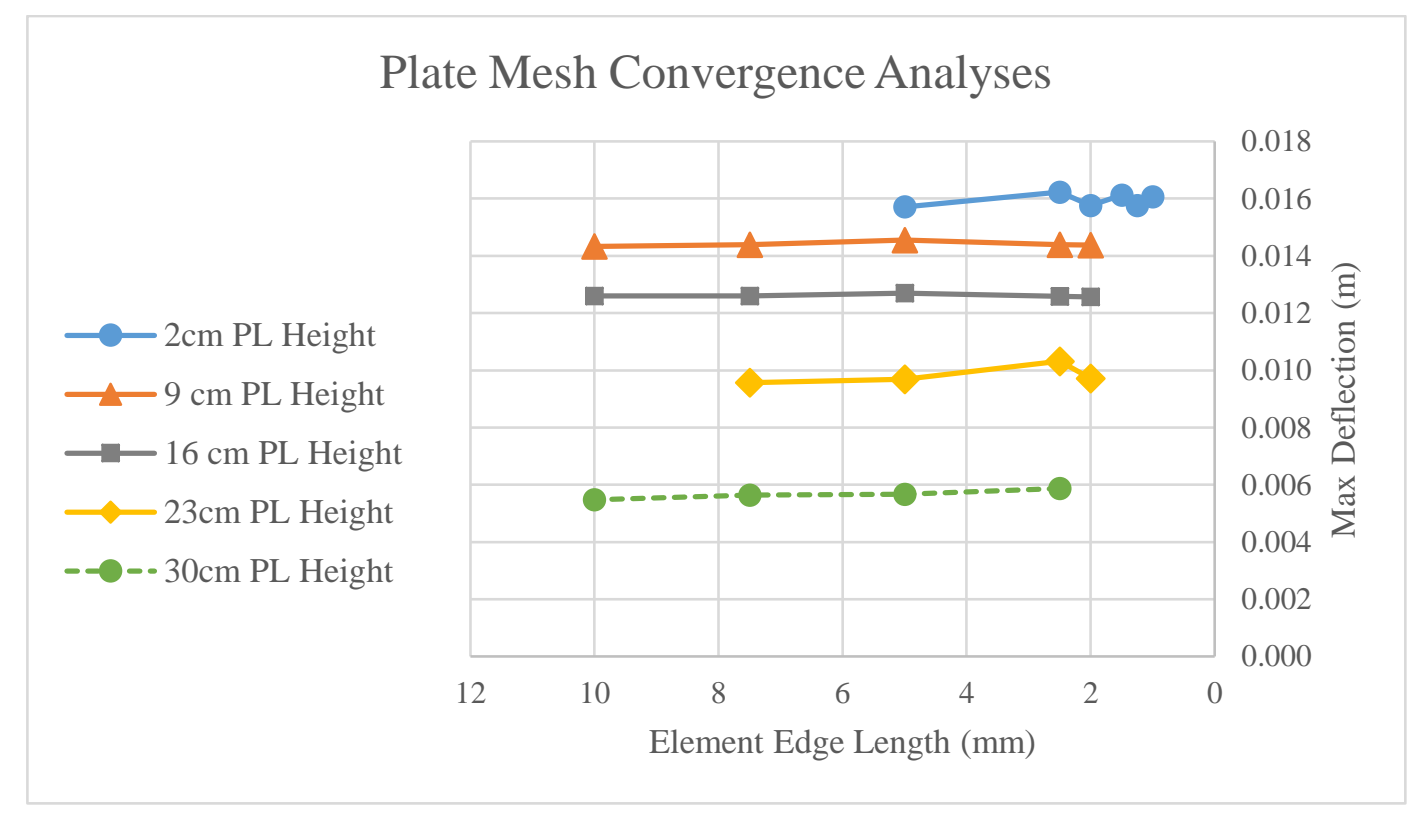

Figure 3.12 Plate Models Mesh Convergence Analyses

\subsubsection{Creating the Plate Meta-Model}

Once the FEA models are computed, the meta-model can be created. First, for each patch load height, the load to cause yield at the edges and the load to cause yield at the center of the plate are tabulated (Table 3.2) to determine the applied contact force levels (Table 3.3) for both the elastic and plastic response models. As the plate response varies with the size of the patch load, the contact force range must accommodate all the possible plate responses 
for all the patch loads. Therefore, the elastic model will range from a relatively low contact force to the largest load to cause yield at the edges of the plate. Likewise, for the plastic plate response model, the contact force has a low level of the smallest load to cause the plate to yield at the edges, and the highest load to cause collapse. The wide variation in the load to cause yield depending on the patch load height is also why accurate transition and termination models are required so that the model response does not continue past where it is applicable for that patch load height.

Table 3.2 Loads to Cause Edge and Centre Yield

\begin{tabular}{|r|r|r|}
\hline $\begin{array}{c}\text { Patch Load Height } \\
(\mathrm{cm})\end{array}$ & $\begin{array}{c}\text { Applied Load to Cause } \\
\text { Yield at Edges (N) }\end{array}$ & $\begin{array}{c}\text { Applied Load to Cause } \\
\text { Centre Hinge Collapse (N) }\end{array}$ \\
\hline 2 & 256,780 & 260,792 \\
\hline 9 & 292,900 & 298,250 \\
\hline 16 & 337,054 & 349,092 \\
\hline 23 & 389,231 & 418,657 \\
\hline 30 & 477,452 & 522,656 \\
\hline
\end{tabular}

Table 3.3 Plate Meta-Model Experimental Levels

\begin{tabular}{|c|c|c|c|c|c|}
\hline \multicolumn{6}{|c|}{ Elastic Response } \\
\hline Levels (5) & 1 & 2 & 3 & 4 & 5 \\
\hline Contact Force, $A(\mathrm{~N})$ & 20,000 & 135,000 & 250,000 & 365,000 & 480,000 \\
\hline Patch Load Height, $B(\mathrm{~cm})$ & 2 & 9 & 16 & 23 & 30 \\
\hline \multicolumn{6}{|c|}{ Plastic Response } \\
\hline Levels (5) & 1 & 2 & 3 & 4 & 5 \\
\hline Contact Force, $A(\mathrm{~N})$ & 250,000 & 318,750 & 387,500 & 456,250 & 525,000 \\
\hline Patch Load Height, $B(\mathrm{~cm})$ & 2 & 9 & 16 & 23 & 30 \\
\hline
\end{tabular}

From there, the collected experimental data (Table 3.4) is input into a regression equation program. In this case, the software Design-Expert 11 [61] is used to compute the experimental results by inputting the results into a historical data type design. Finally, the model fit is assessed and formed to allow for the best prediction capabilities. 
Table 3.4 Plate Meta-Model Experimental Runs

\begin{tabular}{|r|r|r|r|}
\hline \multicolumn{4}{|c|}{ Elastic Response } \\
\hline & \multicolumn{2}{|c|}{ Factors } & \multicolumn{1}{c|}{ Solutions } \\
\cline { 2 - 4 } & $\begin{array}{l}\text { Contact } \\
\text { Force, } A \\
(\mathrm{~N})\end{array}$ & $\begin{array}{l}\text { Patch Load } \\
\text { Height, } B \\
(\mathrm{~cm})\end{array}$ & $\begin{array}{l}\text { Internal } \\
\text { Energy } \\
(\mathrm{J})\end{array}$ \\
\hline 1 & 155,000 & 2 & 1747.03 \\
\hline 2 & 200,000 & 30 & 487.88 \\
\hline 3 & 200,000 & 23 & 1119.41 \\
\hline 4 & 200,000 & 16 & 1936.35 \\
\hline 5 & 20,000 & 23 & 0.42 \\
\hline 6 & 155,000 & 23 & 425.56 \\
\hline 7 & 20,000 & 9 & 0.82 \\
\hline 8 & 155,000 & 9 & 1301.40 \\
\hline 9 & 65,000 & 30 & 11.73 \\
\hline 10 & 65,000 & 16 & 28.06 \\
\hline 11 & 20,000 & 16 & 0.62 \\
\hline 12 & 110,000 & 16 & 151.07 \\
\hline 13 & 200,000 & 2 & 3020.21 \\
\hline 14 & 110,000 & 9 & 280.88 \\
\hline 15 & 110,000 & 23 & 86.19 \\
\hline 16 & 65,000 & 9 & 37.36 \\
\hline 17 & 20,000 & 2 & 0.96 \\
\hline 18 & 110,000 & 30 & 42.36 \\
\hline 19 & 110,000 & 2 & 479.61 \\
\hline 20 & 155,000 & 159.68 \\
\hline 21 & 20,000 & 30 & 0.26 \\
\hline 22 & 200,000 & 2596.65 \\
\hline 23 & 65,000 & 43.51 \\
\hline 24 & 65,000 & 18.85 \\
\hline 25 & 155,000 & 759.40 \\
\hline
\end{tabular}

\begin{tabular}{|c|c|c|c|}
\hline \multicolumn{4}{|c|}{ Plastic Response } \\
\hline & \multicolumn{2}{|c|}{ Factors } & Solutions \\
\hline & Contact & Patch Load & Internal \\
\hline & Force, $A$ & Height, $B$ & Energy \\
\hline & & & \\
\hline 1 & 253,500 & 2 & 2755.36 \\
\hline 2 & 300,000 & 30 & 686.64 \\
\hline 3 & 300,000 & 23 & 1510.27 \\
\hline 4 & 300,000 & 16 & 2396.19 \\
\hline 5 & 114,000 & 23 & 86.19 \\
\hline 6 & 253,500 & 23 & 930.54 \\
\hline 7 & 114,000 & 9 & 280.88 \\
\hline 8 & 253,500 & $\overline{9}$ & 2344.84 \\
\hline 9 & 160,500 & 30 & 94.72 \\
\hline 10 & 160,500 & 16 & 458.11 \\
\hline 11 & 114,000 & 16 & 151.07 \\
\hline 12 & 207,000 & 16 & 960.26 \\
\hline 13 & 300,000 & 2 & 3550.23 \\
\hline 14 & 207,000 & 9 & 1579.79 \\
\hline 15 & 207,000 & 23 & 528.33 \\
\hline 16 & 160,500 & 9 & 767.93 \\
\hline 17 & 114,000 & 2 & 479.61 \\
\hline 18 & 207,000 & 30 & 208.06 \\
\hline 19 & 207,000 & 2 & 2010.68 \\
\hline 20 & 253,500 & 30 & 401.39 \\
\hline 21 & 114,000 & 30 & 42.36 \\
\hline 22 & 300,000 & 9 & 3049.64 \\
\hline 23 & 160,500 & 2 & 1168.46 \\
\hline 24 & 160,500 & 23 & 226.96 \\
\hline 25 & 253,500 & 16 & 1686.05 \\
\hline
\end{tabular}

To examine the models' fit, the following residual analyses are conducted through the design of experiments software. Box-Cox plot and the normal plot of residuals are used to determine if the data requires a transformation. For the elastic and plastic response, for this case, the analysis suggested that the models require a natural log transformation. 


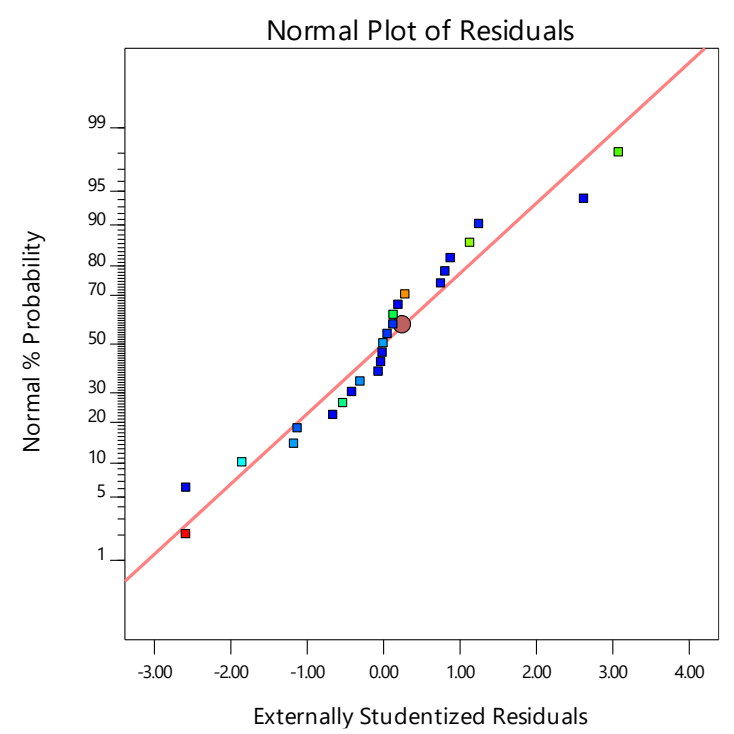

Figure 3.13 Normal Plot of Residuals Elastic Plate Response No Transformation

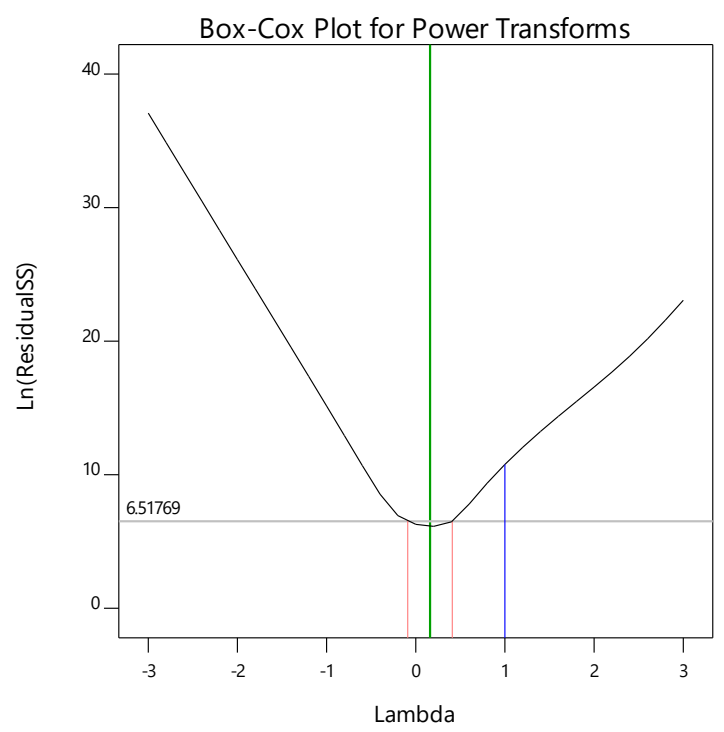

Figure 3.15 Box-Cox Plot - Elastic Plate Response No Transformation

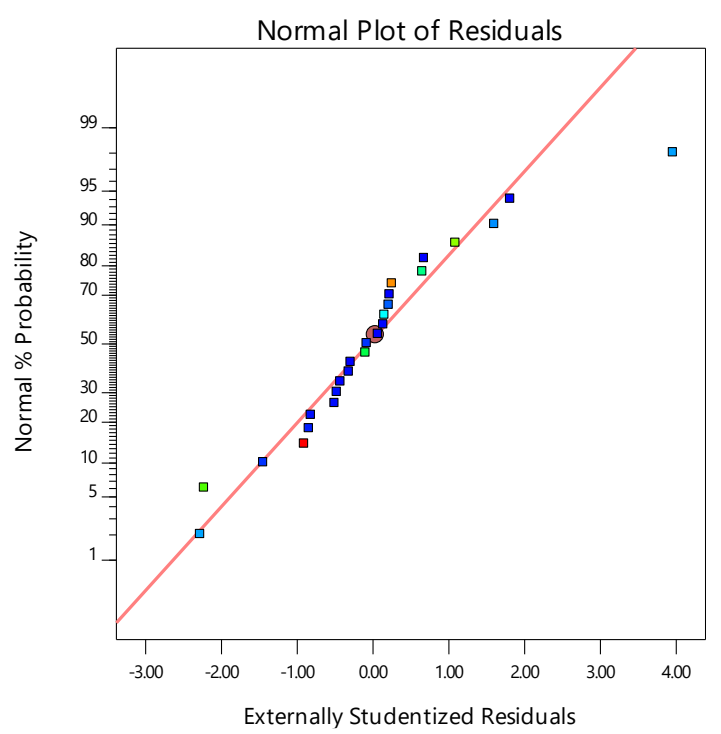

Figure 3.14 Normal Plot of Residuals Elastic Plate Response Natural Log Transformation

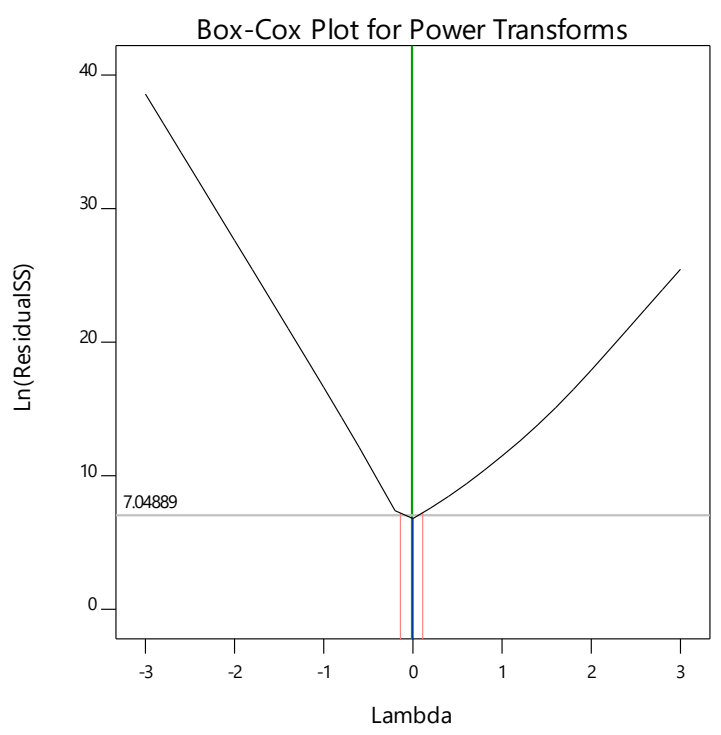

Figure 3.16 Box-Cox Plot - Elastic Plate Response Natural Log Transformation 


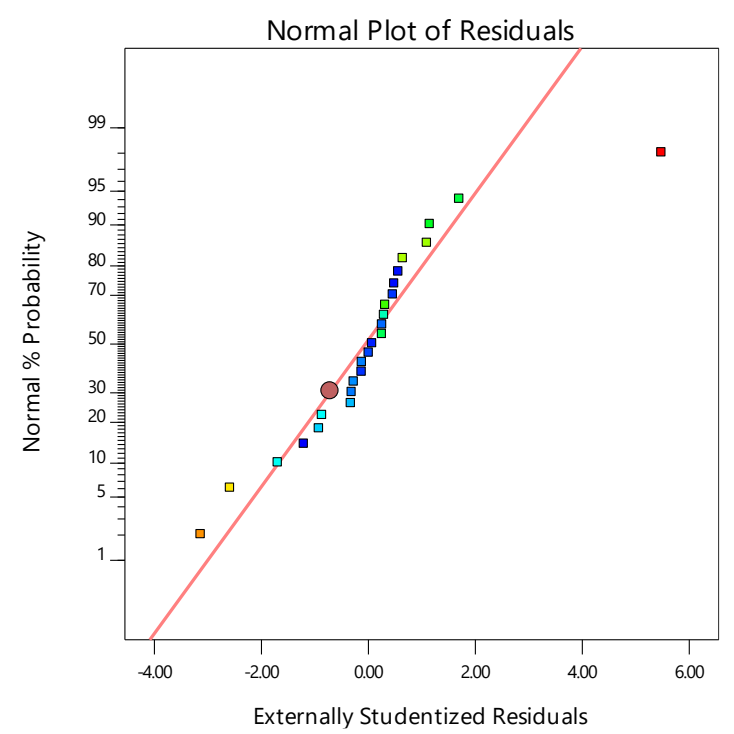

Figure 3.17 Normal Plot of Residuals Plastic Plate Response No Transformation

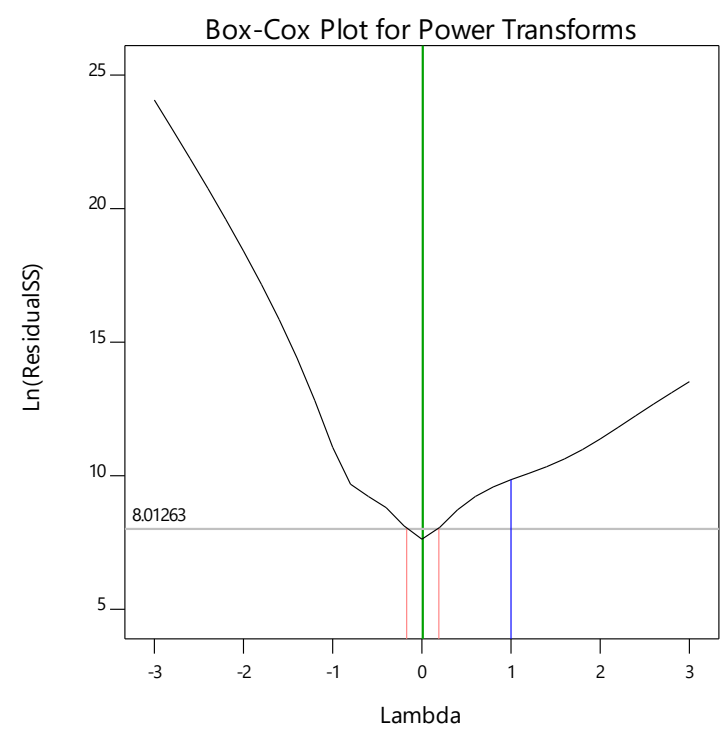

Figure 3.19 Box-Cox Plot - Plastic Plate Response No Transformation

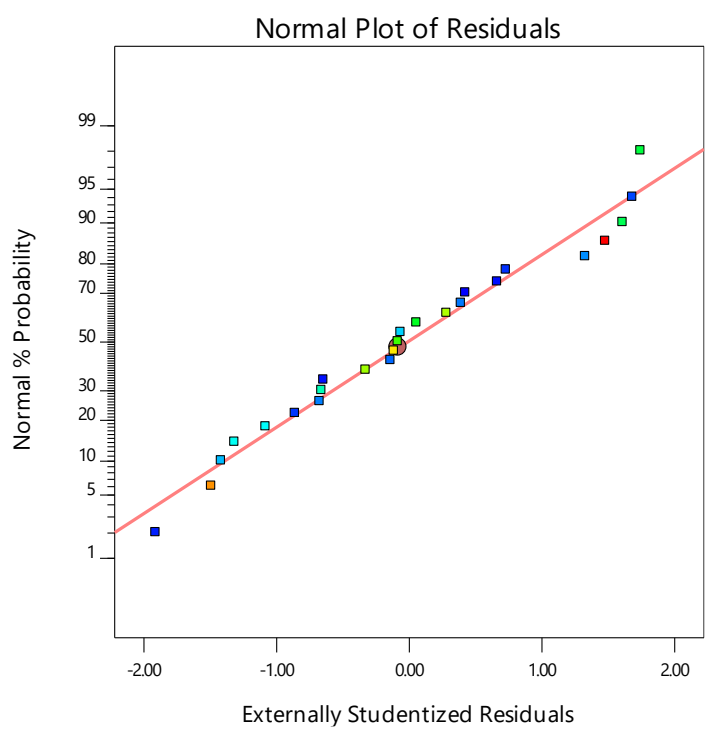

Figure 3.18 Normal Plot of Residuals Plastic Plate Response Natural Log Transformation

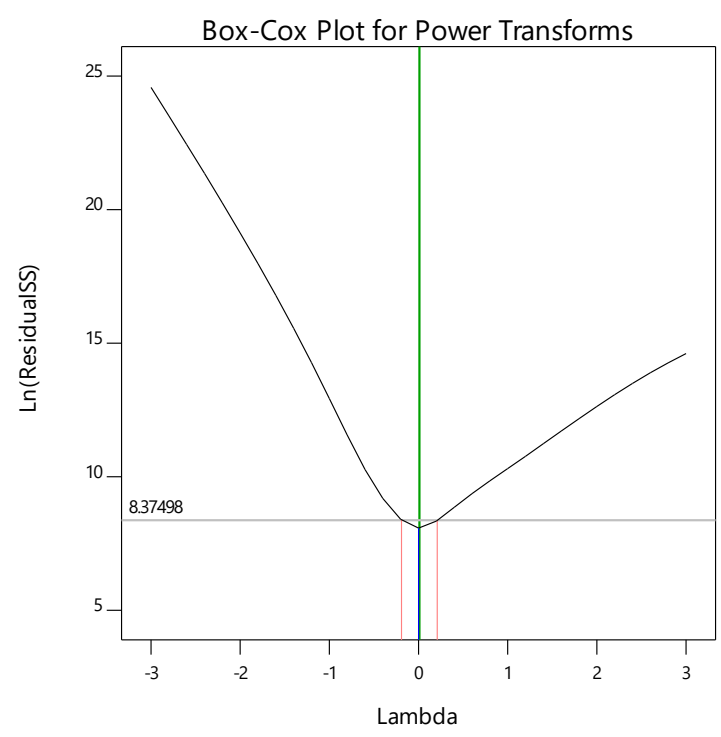

Figure 3.20 Box-Cox Plot - Plastic Plate Response Natural Log Transformation 
The analysis of variance (ANOVA) and fit statistic $\mathrm{R}^{2}$ are used to assess the significance of the factor and interaction terms. These analyses are conducted to simplify the model by removing any insignificant terms, without disturbing the models' hierarchy. If any term has a p-value $<0.0001$ the term is significant, and the larger the F-value the more significant the term is. For the elastic model, the majority of the higher-order $B$ (patch load height) terms are removed, leaving $A^{4}$ (Contact Force), $A^{3} B, A^{3}$, and $A^{2} B \cdot A^{4}, A^{3} B$, and $A^{2} B$ are left in the model since they have a slight significance and help the model fit. With the removal of some of the higher-order interaction terms, the fit significance is checked to make sure the fit has improved or at least not worsened. With the removal of most of the higher-order $B$ terms, the fit analysis (Table 3.6) indicates good agreement between the adjusted and predicted $\mathrm{R}^{2}$ and a high precision ratio term (Adeq Precision), compared to the minimum allowable value of 4 .

Table 3.5 ANOVA Analysis of Elastic Plate Model

\begin{tabular}{|l|r|r|r|r|r|r|}
\hline & \multicolumn{3}{|c|}{ Full Set of Terms } & \multicolumn{3}{c|}{ Adjusted Terms } \\
\hline Source & F-value & p-value & & F-value & p-value & \\
\hline Model & 1477.74 & $<0.0001$ & significant & 2075.01 & $<0.0001$ & significant \\
\hline A (Contact Force) & 1125.99 & $<0.0001$ & & 1126.93 & $<0.0001$ & \\
\hline $\begin{array}{l}\text { B (Patch Load } \\
\text { Height) }\end{array}$ & 132.05 & $<0.0001$ & & 540.46 & $<0.0001$ & \\
\hline $\mathrm{AB}$ & 12.33 & 0.0056 & & 26.37 & 0.0001 & \\
\hline $\mathrm{A}^{2}$ & 0.4563 & 0.5147 & & 0.8678 & 0.3663 & \\
\hline $\mathrm{B}^{2}$ & 0.0715 & 0.7946 & & 29.88 & $<0.0001$ & \\
\hline $\mathrm{A}^{2} \mathrm{~B}$ & 22.98 & 0.0007 & & 20.75 & 0.0004 & \\
\hline $\mathrm{AB}^{2}$ & 3.90 & 0.0764 & & & & \\
\hline $\mathrm{A}^{3}$ & 98.69 & $<0.0001$ & & 89.12 & $<0.0001$ & \\
\hline $\mathrm{B}^{3}$ & 0.1166 & 0.7398 & & & & \\
\hline $\mathrm{A}^{2} \mathrm{~B}^{2}$ & 2.04 & 0.1841 & & & & \\
\hline $\mathrm{A}^{3} \mathrm{~B}$ & 15.89 & 0.0026 & & 14.34 & 0.0018 & \\
\hline $\mathrm{AB}^{3}$ & 0.3759 & 0.5535 & & & & \\
\hline $\mathrm{A}^{4}$ & 29.38 & 0.0003 & & 26.53 & 0.0001 & \\
\hline $\mathrm{B}^{4}$ & 0.1794 & 0.6808 & & & & \\
\hline
\end{tabular}


Table 3.6 Fit Statistic $\mathrm{R}^{2}$ Elastic Plate Model

\begin{tabular}{|l|r|r|}
\hline & Full Set of Terms & Adjusted Terms \\
\hline $\mathrm{R}^{\mathbf{2}}$ & 0.9995 & 0.9992 \\
\hline Adjusted $\mathrm{R}^{\mathbf{2}}$ & 0.9988 & 0.9987 \\
\hline Predicted $\mathrm{R}^{\mathbf{2}}$ & 0.9959 & 0.9969 \\
\hline Adeq Precision & 119.58 & 141.03 \\
\hline
\end{tabular}

A similar analysis is conducted with the plastic response model. In this case, half the quartic terms are removed to help increase the fit of the model. A few less significant terms, with higher p-values, remain in the model to ensure the model follows the hierarchical rules. With the removal of the more insignificant terms, both the precision ratio and the predicted $\mathrm{R}^{2}$ term increased, suggesting an improved model fit.

Table 3.7 ANOVA Analysis Plastic Model

\begin{tabular}{|l|r|r|r|r|r|r|}
\hline & \multicolumn{3}{|c|}{ Full Set of Terms } & \multicolumn{3}{c|}{ Adjusted Terms } \\
\hline Source & F-value & p-value & & F-value & p-value & \\
\hline Model & 5342.36 & $<0.0001$ & significant & 5626.89 & $<0.0001$ & significant \\
\hline A (Contact Force) & 4146.28 & $<0.0001$ & & 3431.56 & $<0.0001$ & \\
\hline $\begin{array}{l}\text { B (Patch Load } \\
\text { Height) }\end{array}$ & 2846.04 & $<0.0001$ & & 2355.45 & $<0.0001$ & \\
\hline $\mathrm{AB}$ & 100.11 & $<0.0001$ & & 124.14 & $<0.0001$ & \\
\hline $\mathrm{A}^{2}$ & 79.54 & $<0.0001$ & & 717.92 & $<0.0001$ & \\
\hline $\mathrm{B}^{2}$ & 31.81 & 0.0002 & & 567.28 & $<0.0001$ & \\
\hline $\mathrm{A}^{2} \mathrm{~B}$ & 74.29 & $<0.0001$ & & 61.49 & $<0.0001$ & \\
\hline $\mathrm{AB}^{2}$ & 127.52 & $<0.0001$ & & 105.54 & $<0.0001$ & \\
\hline $\mathrm{A}^{3}$ & 11.90 & 0.0062 & & 9.85 & 0.0078 & \\
\hline $\mathrm{B}^{3}$ & 11.75 & 0.0065 & & 9.72 & 0.0082 & \\
\hline $\mathrm{A}^{2} \mathrm{~B}^{2}$ & 47.20 & $<0.0001$ & & 39.06 & $<0.0001$ & \\
\hline $\mathrm{A}^{3} \mathrm{~B}$ & 16.37 & 0.0023 & & 13.55 & 0.0028 & \\
\hline $\mathrm{AB}^{3}$ & 2.55 & 0.1416 & & & & \\
\hline $\mathrm{A}^{4}$ & 1.67 & 0.2254 & & & & \\
\hline $\mathrm{B}^{4}$ & 1.49 & 0.2499 & & & & \\
\hline
\end{tabular}


Table 3.8 Fit Statistic $\mathrm{R}^{2}$ Plastic Model

\begin{tabular}{|l|r|r|}
\hline & Full Set of Terms & Adjusted Terms \\
\hline $\mathrm{R}^{\mathbf{2}}$ & 0.9999 & 0.9998 \\
\hline Adjusted $\mathrm{R}^{\mathbf{2}}$ & 0.9997 & 0.9996 \\
\hline Predicted $\mathrm{R}^{\mathbf{2}}$ & 0.9992 & 0.9989 \\
\hline Adeq Precision & 260.02 & 264.48 \\
\hline
\end{tabular}

Finally, two meta-models are created to model the elastic and plastic deformation energy. Both the elastic deformation energy (Eq. 35) and the plastic deformation energy (Eq. 36) are dependent on the patch load height $\left(h_{p l}\right)$ in meters and the contact force $\left(F_{n}\right)$ in newtons.

$$
\begin{aligned}
S E_{p_{e}}=\exp ( & -1.15865+0.000058 \cdot F_{n}-0.311354 \cdot h_{p l}+0.000017 \\
& \cdot F_{n} h_{p l}-2.14826 * 10^{-10} \cdot F_{n}{ }^{2}-14.10722 \cdot h_{p l}{ }^{2}-1.91252 \\
& * 10^{-10} \cdot F_{n}{ }^{2} h_{p l}+5.12962 * 10^{-16} \cdot F_{n}{ }^{3}+3.13667 * 10^{-16} \\
& \left.\cdot F_{n}{ }^{3} h_{p l}-4.85752 * 10^{-22} \cdot F_{n}{ }^{4}\right) \\
S E_{p_{p}}=\exp ( & -2.14381+0.000057 \cdot F_{n}-13.01753 \cdot h_{p l}-0.000032 \\
& \cdot F_{n} h_{p l}-1.13710 * 10^{-10} \cdot F_{n}{ }^{2}+84.47672 \cdot h_{p l}{ }^{2}+2.77639 \\
& * 10^{-10} \cdot F_{n}{ }^{2} h_{p l}-0.000423 \cdot F_{n} h_{p l}{ }^{2}+7.94952 * 10^{-17} \cdot F_{n}{ }^{3} \\
& -25.84532 \cdot h_{p l}{ }^{3}+4.64974 * 10^{-10} \cdot F_{n}{ }^{2} h_{p l}{ }^{2}-3.25260 \\
& \left.* 10^{-16} \cdot F_{n}{ }^{3} h_{p l}\right)
\end{aligned}
$$

\subsubsection{Transition and Termination Models}

Transition and termination models are also established to determine the appropriate material response, depending on the contact force and patch load height. The transition model finds the load at which the plate will yield at the edges, indicating when the algorithm 
switches from the elastic model to the plastic model. The termination model predicts the contact force that will surpass the limit state of centre hinge collapse, modelled as full through-thickness yield at the center of the plate.

The transition and termination models for the plate material response models have only one input, patch load height, and one output, the contact force. Therefore, these models only have one factor with five levels, and consequently, only require an experiment with five runs (Table 3.9). As the experiment only has five runs, only up to a cubic form can be modelled to allow for some pure error. If a higher-order form is desired, more factor levels and or runs are required.

Table 3.9 Plate Transition and Termination Experimental Levels and Runs

\begin{tabular}{|r|r|r|r|}
\hline \multicolumn{1}{|c|}{ Fun } & Factor 1 & \multicolumn{1}{c|}{ Response 1 } & \multicolumn{1}{c|}{ Response 2 } \\
& Patch Load Height (cm) & Load to Initial Yield (N) & $\begin{array}{c}\text { Load to center hinge } \\
\text { collapse (N) }\end{array}$ \\
\hline 1 & 2 & 256,780 & 260,792 \\
\hline 2 & 9 & 292,900 & 298,250 \\
\hline 3 & 16 & 337,054 & 349,092 \\
\hline 4 & 23 & 389,231 & 418,657 \\
\hline 5 & 30 & 477,452 & 522,656 \\
\hline
\end{tabular}

The experimental data for the transition and termination models is evaluated similarly to the material response models with the software Design-Expert 11 [61] to create the regression equations and implement similar prediction capability assessments. The normal plot of residuals and Cox-Box plots are used to determine if transformations are required. Examining the ANOVA tables and fit statistic $\mathrm{R}^{2}$ is then followed to assess the significant factors to include in the meta-models. Both equations identify the contact force, in newtons, for the load to cause yield at the edges of the plate (Eq. 37) and the centre of the plate (Eq. 38) dependent on the patch load height $\left(h_{p l}\right)$ in meters. 


$$
\begin{aligned}
& Y_{E d}=\left(4.00376 * 10^{-6}-6.34975 * 10^{-6} \cdot h_{p l}\right)^{-1} \\
& Y_{C H}=\left(3.96942 * 10^{-6}-6.86662 * 10^{-6} \cdot h_{p l}\right)^{-1}
\end{aligned}
$$

Eq. 38 


\section{Chapter 4 Algorithm Benchmarking}

Various types of benchmarking analyses are conducted to assess the capabilities of the algorithm, through the use of FEA. The individual structural models are assessed, in addition to examining the response of the algorithm compared to an FEA grillage structure. The grillage structure is also implemented to investigate the assumption of a rectangular patch load against the contact geometry that is produced from an ice wedge normal to the hull interaction.

\subsection{Individual Structural Models Analyses}

As the structural response models are developed, the individual models are evaluated before they are placed into the algorithm. The beam model is developed as an analytical model, therefore FEA is applied to conduct the benchmarking. Since the plate model is developed through the analysis of multiple FEA models, FEA can still be used to benchmark the derived meta-model.

\subsubsection{Beam}

To examine the quality of the analytical beam model, benchmarking is conducted through the use of FEA. Three different loading conditions are analyzed to assess if there is any variation in the quality of the results with the change of the length of the patch load. A full uniformly loaded beam, a patch load that is half the length of the beam, and a patch load a quarter the length of the beam are investigated. A 1.5 meter long beam is created in FEA with beam elements, and a $180 \mathrm{~mm}$ thick by $9 \mathrm{~mm}$ wide rectangular cross-section (Figure 
4.1). The beam is fixed on one end and restrained to only translate longitudinally on the other end, to create a fixed but free-to-pull in boundary condition.

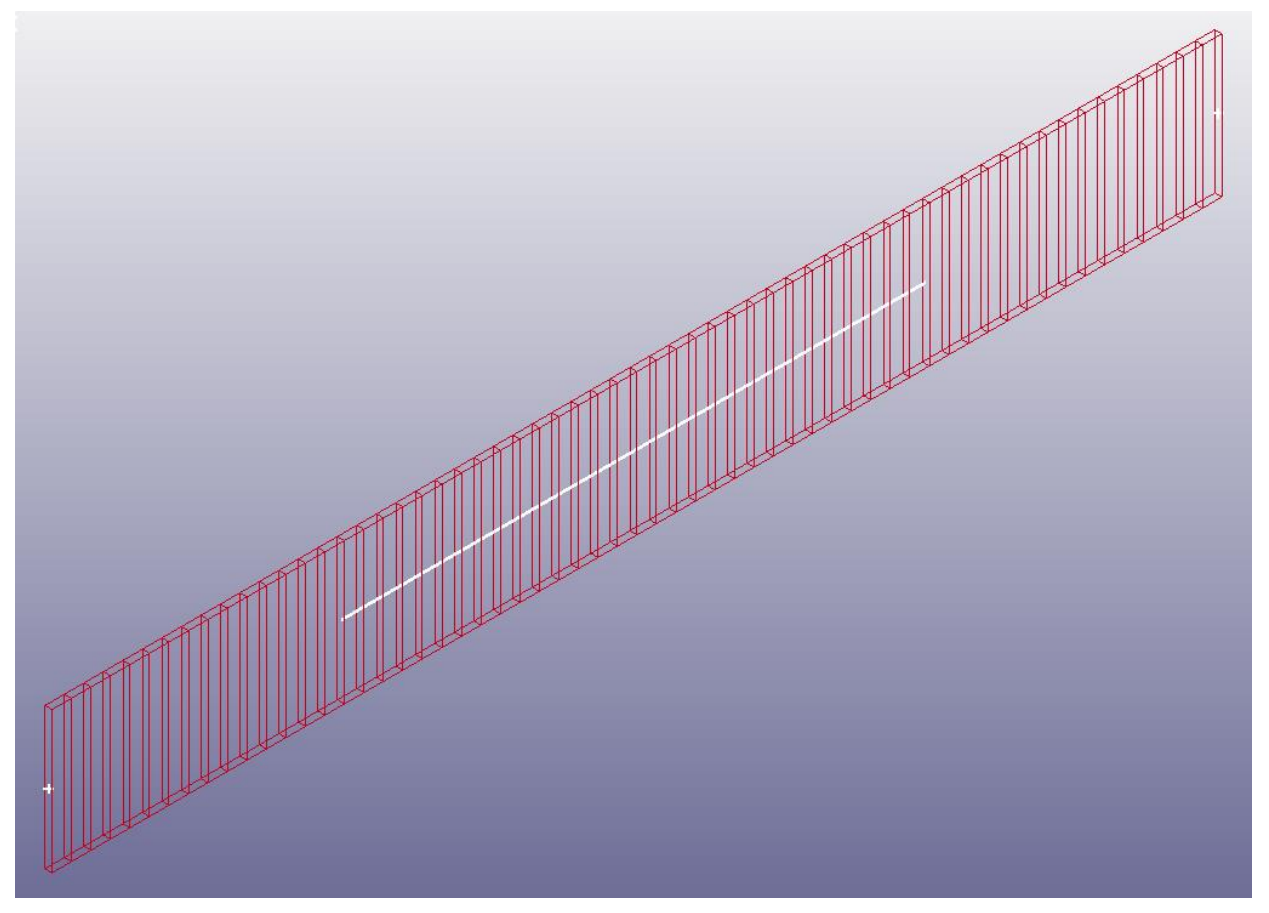

Figure 4.1 FEA Isolated Beam Model (Half-length patch load)

For the material model, an elastic perfectly plastic material is implemented (Figure 4.2), with the material properties of structural steel, which has a density of $7850 \mathrm{~kg} / \mathrm{m}^{3}$, Young's modulus of $200 \mathrm{GPa}$, a Poisson's ratio of 0.3 , a yield strength of $250 \mathrm{MPa}$, and an $E_{T a n}$ of $0 \mathrm{MPa}$. The FEA models are created with similar assumptions to the analytical model, to allow for only the modelling capabilities of the analytical model to be assessed. Similar to the FEA plate models, a uniform beam load is applied to a set of beam segments with a unit ramp (Figure 3.11b), to create a static loading event and remove any inertial effects from the beam response. For the full beam length patch load, a distributed load of $191.5 \mathrm{kN} / \mathrm{m}$ is applied across the beam elements; for the half beam length patch load, $220 \mathrm{kN} / \mathrm{m}$ is applied 
across the patch; and for the quarter beam length patch load, $375 \mathrm{kN} / \mathrm{m}$ are applied across the patch.

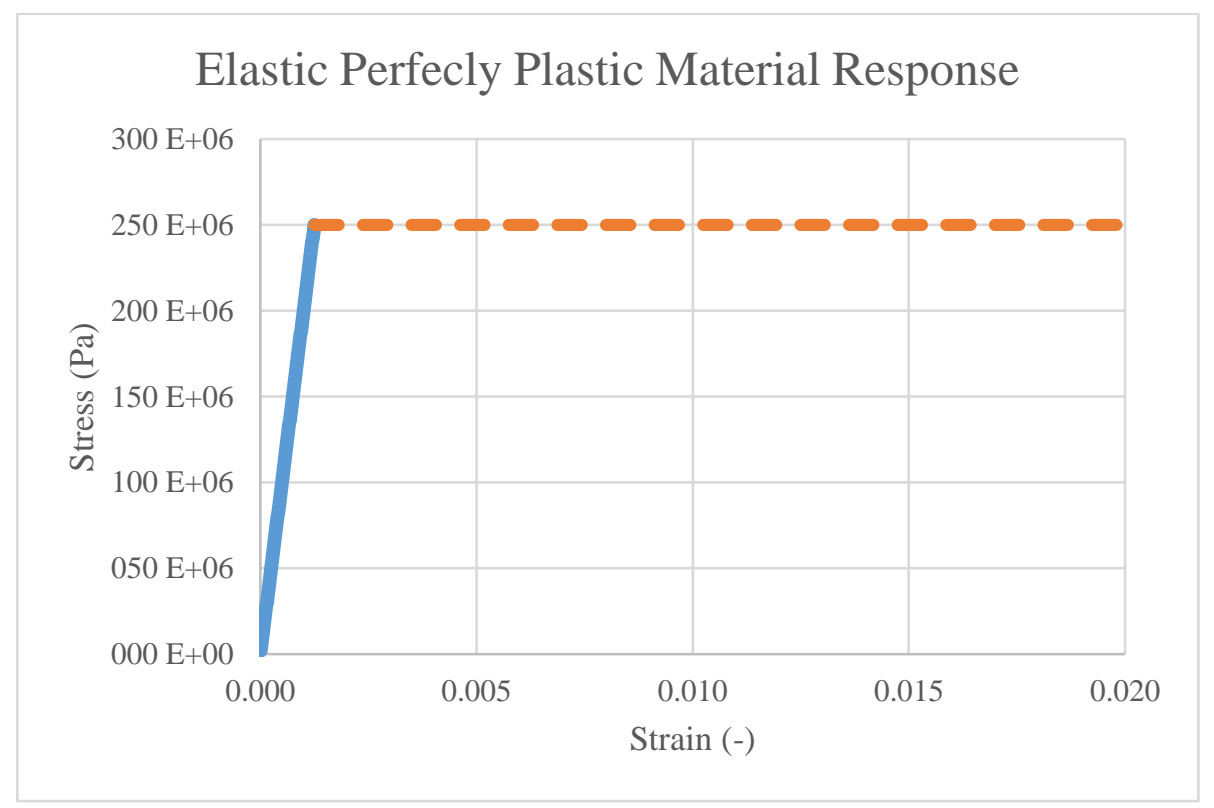

Figure 4.2 Elastic Perfectly Plastic Material Model

Once the FEA models are created, mesh convergence analyses (Figure 4.3) are conducted for each of the three loading conditions, to confirm that the mesh elements are appropriately sized to model the true response. The FEA models in Figure 4.3 compare the deflection at the center of the beam, at the load to cause yield at the ends of the beam. For the uniform load across the full length of the beam, the response appears to have started to settle at around 80 elements, with only a $0.05 \mathrm{~mm}$ difference in displacement compared to the 100 element beam. For both the half- and quarter-length beam patch loads, again, 80 beam elements were chosen to model the beam response. The half-length patch load settles at around 80 elements with only a $0.03 \mathrm{~mm}$ difference with each 20 element increase. The quarter-length patch load has very little difference in the deflection with only a maximum 
increase of $0.06 \mathrm{~mm}$ with each additional 40 elements. Therefore, 80 elements were chosen as a conservative model to make sure the true response is modelled.

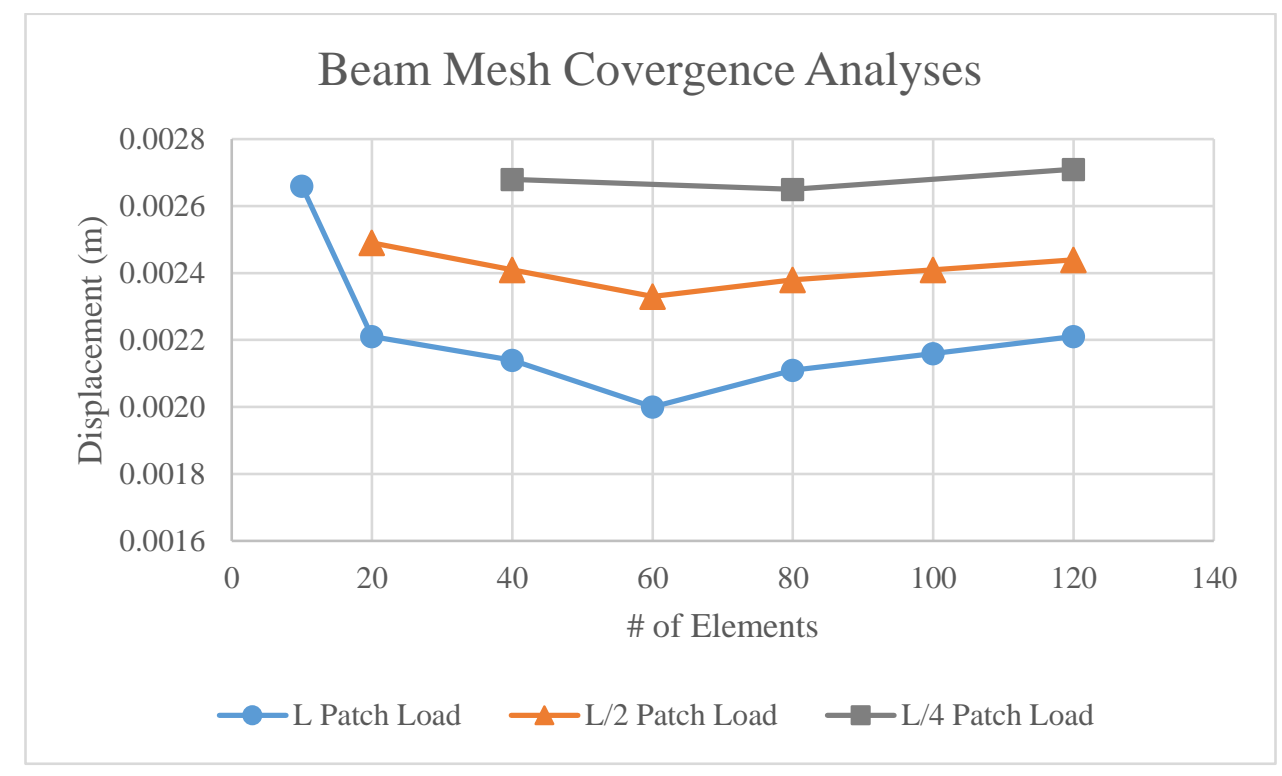

Figure 4.3 Beam FEA Benchmarking - Mesh Convergence Analyses

\subsubsection{Analytical Model Analysis}

First, the analytical beam model is examined visually, and compared to the FEA internal energy responses, Figure 4.4 to Figure 4.6. The elastic response of the strain bending energy model has very good agreement with all the FEA models; the plastic response does not have the same level of agreement as the elastic response. In all three cases, it can be observed that the analytical model transitions from the elastic to the plastic response and terminates before the FEA models. In addition, as the patch loads decrease in length, the range of the plastic response also shrinks, which does reflect the same observable trends of the FEA models. But as the patch load length decreases, the analytical plastic model terminates at an increased rate compared to the larger patch loads. For example, the plastic 
response of the smallest patch load, a quarter-length of the beam, terminates before the FEA model even enters the plastic response.

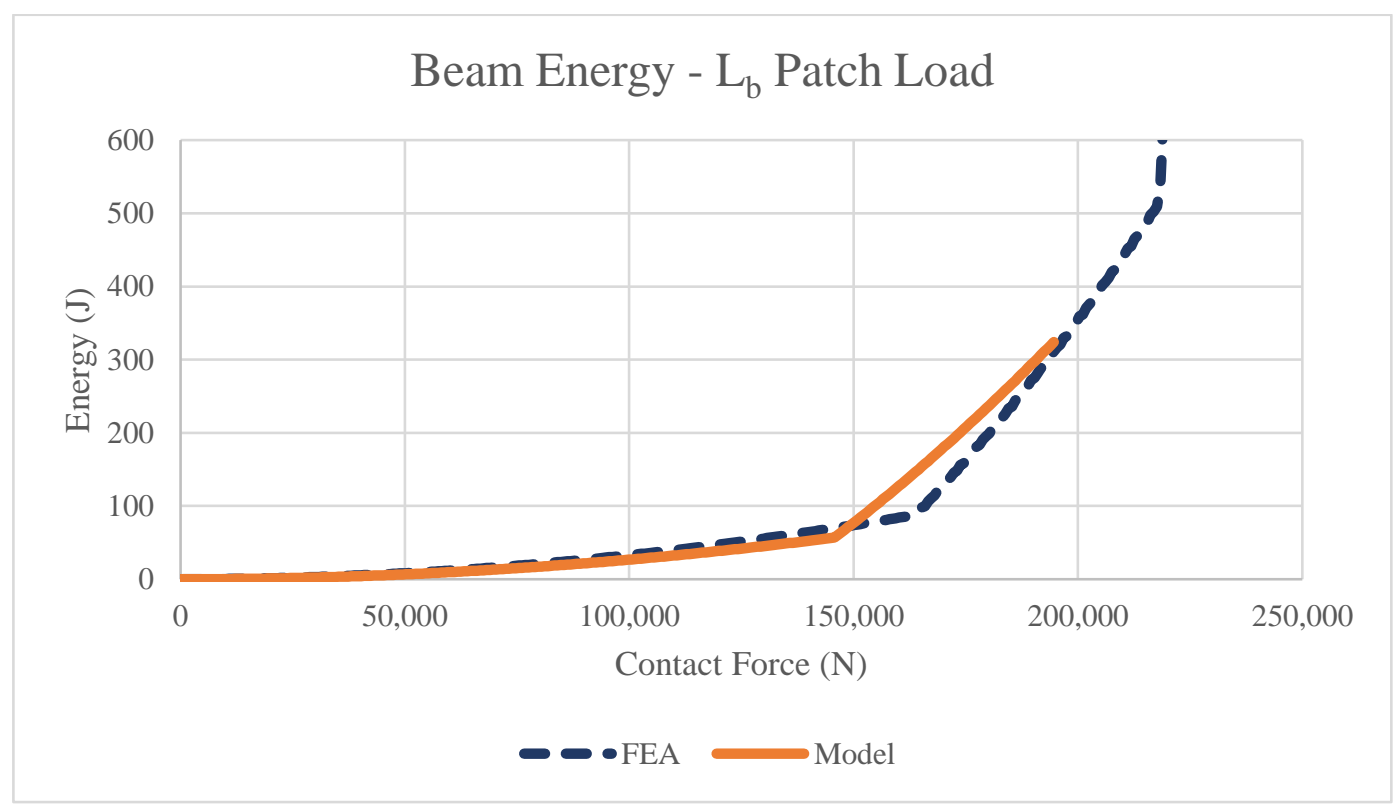

Figure 4.4 Uniformly Loaded Beam - Energy Analysis

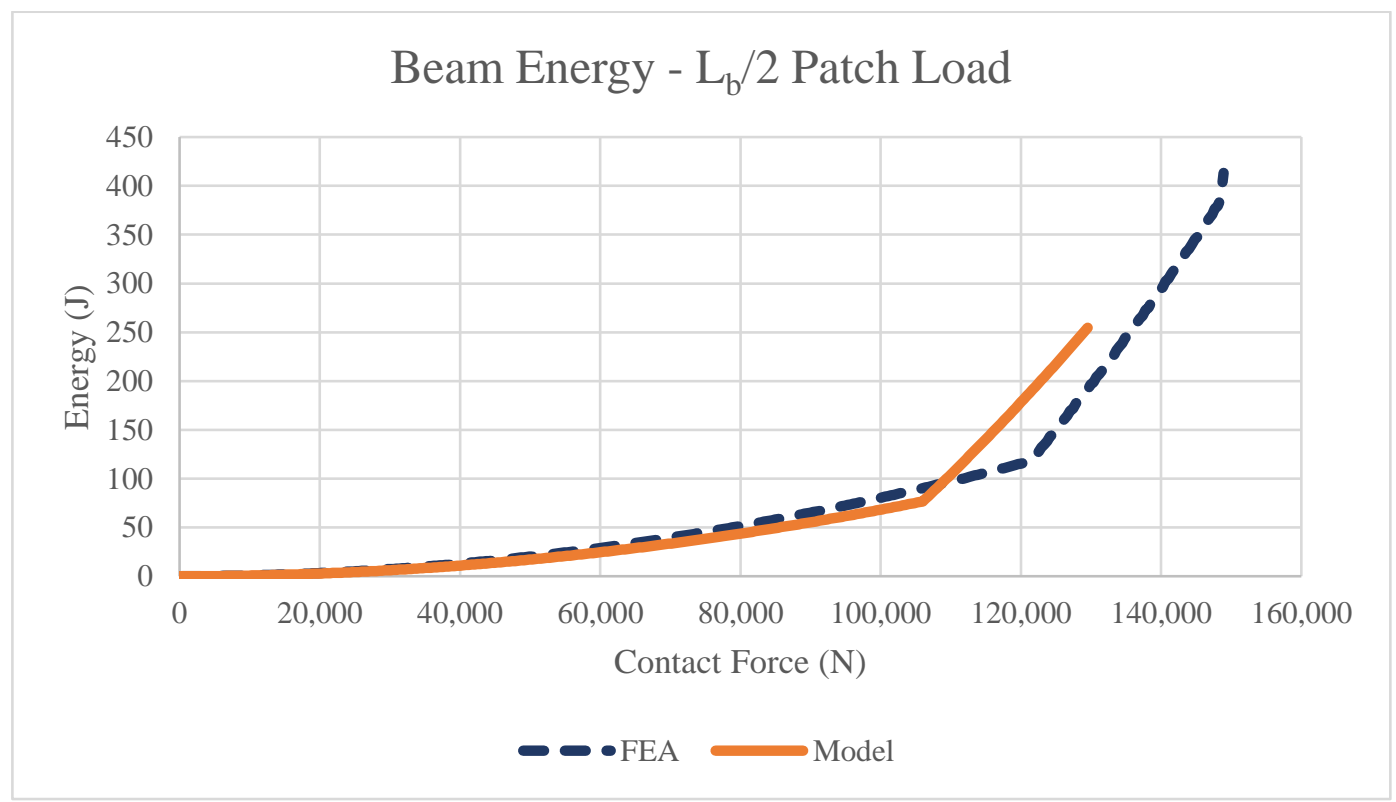

Figure 4.5 Beam Half-Length Patch Load - Energy Analysis 


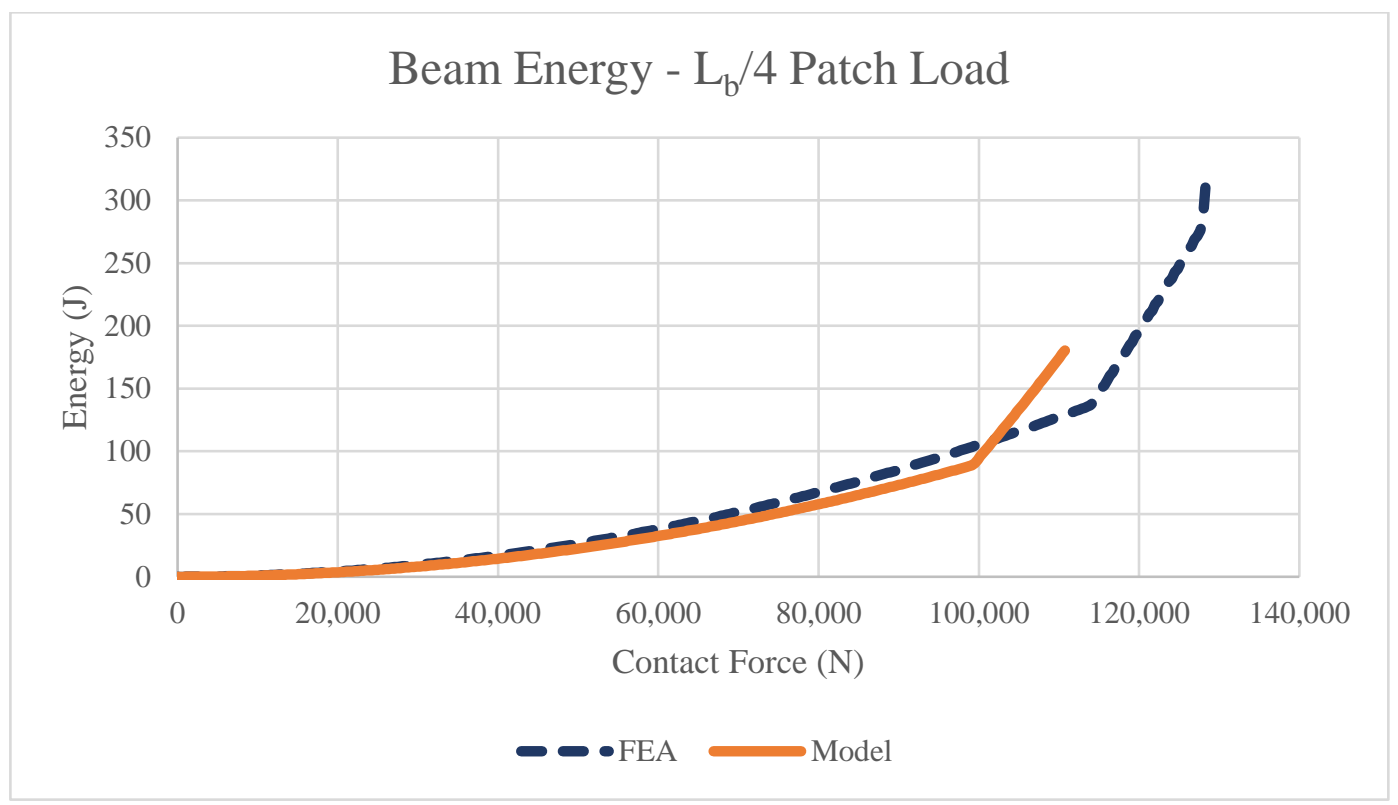

Figure 4.6 Beam Quarter-Length Patch Load - Energy Analysis

After the initial visual assessment, the beam model's transition and termination loads are assessed to see the percent difference between the model and FEA responses, tabulated in Table 4.1. Both the loads to cause yield at the ends and centre of the beam are underpredicted compared to the FEA analyses, with an average percent difference between the model and FEA at $12.3 \%$, and all under $15 \%$.

Table 4.1 Beam - Transition and Termination Loads

\begin{tabular}{|c|c|c|c|c|}
\hline \multirow[b]{2}{*}{$\begin{array}{c}\text { Yield } \\
\text { Location }\end{array}$} & \multirow{2}{*}{$\begin{array}{c}\text { Patch Load Length } \\
\text { (where } \mathrm{L}_{b}=\text { Length of } \\
\text { Beam) }\end{array}$} & \multicolumn{2}{|c|}{ Transition Forces $(\mathrm{N})$} & \multirow[b]{2}{*}{$\begin{array}{c}\text { Percent } \\
\text { Difference }\end{array}$} \\
\hline & & $\begin{array}{c}\text { Model } \\
\text { Estimation }\end{array}$ & $\begin{array}{c}\text { FEA } \\
\text { Estimation }\end{array}$ & \\
\hline \multirow{3}{*}{ Ends } & $\mathrm{L}_{\mathrm{b}}$ & 145,779 & 163,732 & $11.0 \%$ \\
\hline & $\mathrm{L}_{\mathrm{b}} / 2$ & 106,425 & 121,687 & $12.5 \%$ \\
\hline & $\mathrm{L}_{\mathrm{b}} / 4$ & 99,492 & 114,257 & $12.9 \%$ \\
\hline \multirow{3}{*}{ Center } & $\mathrm{L}_{\mathrm{b}}$ & 193,893 & 216,874 & $10.6 \%$ \\
\hline & $\mathrm{L}_{\mathrm{b}} / 2$ & 129,937 & 149,325 & $13.0 \%$ \\
\hline & $\mathrm{L}_{\mathrm{b}} / 4$ & 111,093 & 128,671 & $13.7 \%$ \\
\hline
\end{tabular}


Also observable in Table 4.1 is that with the decrease in the patch load length there is a slight increase in percent error for both the transition and termination loads. In addition, it can be observed that the termination loads have a slightly larger average error of $12.4 \%$ compared to the average error of $12.1 \%$ of the loads at the transition between the elastic and plastic models.

The collapse energy of the beam, compared in Table 4.2, is significantly under-predicting the maximum energy before the beam reaches the termination limit state of 3-hinge collapse when compared to the FEA internal energy at yield of the center of the beam. Similar to the termination error, the collapse energy prediction error increases with the decrease in the patch load length, which suggests the likely association of the collapse energy error and the termination load of the analytical model. This significant difference in the collapse energy of the model and FEA could be associated with the analytical model terminating before the FEA response and within a response region that is growing significantly with each increase in the contact force, as well as not all of the energy absorption mechanisms being considered in the model. In addition, the termination energy of the analytical model is compared to the traditional simplified 3-hinge collapse model of the three plastic hinge moments and a triangular deflection due to the rotation of the three hinges. When compared to the traditional simplified 3-hinge model, the error is reduced, but still significantly increases with the decrease in the patch load length. 
Table 4.2 Beam - Collapse Energy Comparision

\begin{tabular}{|c|c|c|c|c|c|}
\hline \multirow[b]{2}{*}{$\begin{array}{l}\text { Patch } \\
\text { Load } \\
\text { Length }\end{array}$} & \multicolumn{5}{|c|}{ Termination Energy $(\mathrm{J})$} \\
\hline & $\begin{array}{l}\text { Model } \\
\text { Estimation }\end{array}$ & \begin{tabular}{|l} 
FEA \\
Estimation
\end{tabular} & $\begin{array}{l}\text { Model-FEA } \\
\text { Percent } \\
\text { Difference } \\
\end{array}$ & $\begin{array}{l}\text { Simplified 3- } \\
\text { Hinge Collapse } \\
\text { Estimation }\end{array}$ & $\begin{array}{l}\text { Model-3-Hinge } \\
\text { Percent } \\
\text { Difference }\end{array}$ \\
\hline $\mathrm{L}_{\mathrm{b}}$ & 319.6 & 502.3 & $36.4 \%$ & 376.2 & $15.1 \%$ \\
\hline $\mathrm{L}_{\mathrm{b}} / 2$ & 258.0 & 490.4 & $47.4 \%$ & 335.4 & $23.1 \%$ \\
\hline $\mathrm{L}_{\mathrm{b}} / 4$ & 183.3 & 399.1 & $54.1 \%$ & 273.0 & $32.8 \%$ \\
\hline
\end{tabular}

Finally, statistical analyses of the different beam patch load responses, Table 4.3, are conducted comparing the predicted energies dependent on the applied contact force for both the elastic and plastic responses, within the model's predicted material response ranges. The elastic model has an average percent difference of $16 \%$ across all the patch loads examined, but the errors have very little variance or standard deviation, indicating a consistent error. The plastic models have an average percent difference of around $26 \%$, and a slightly larger variance and standard deviation. The smallest, quarter length, patch load appears to have the best plastic model predictability of the patch load lengths examined, with an average percent difference between the model and FEA of 18\%, but with that said most of the analytical plastic response is being compared to the elastic response of the FEA model. Contrary to the previous transition and termination loads and energy analyses, as the patch load length decreases so does the average error, variance, and standard deviation. This decrease in error is likely influenced by the decrease in the response ranges modelled as the patch load length decreases. In general, the statistical analysis suggests the model has reasonable agreement with the FEA beam models, but the plastic response model terminates too early for the collapse energy to be considered an acceptable agreement. 
Table 4.3 Beam Model Energy Prediciton Statistical Analysis

\begin{tabular}{|c|r|r|r|r|r|r|}
\hline \multirow{2}{*}{$\begin{array}{c}\text { Patch } \\
\text { Load } \\
\text { Length }\end{array}$} & \multicolumn{2}{|c|}{ Mean } & \multicolumn{2}{c|}{ Variance } & \multicolumn{2}{c|}{ Standard Deviation } \\
\cline { 2 - 7 } & $\begin{array}{l}\text { Elastic } \\
\text { Response }\end{array}$ & $\begin{array}{l}\text { Plastic } \\
\text { Response }\end{array}$ & $\begin{array}{l}\text { Elastic } \\
\text { Response }\end{array}$ & $\begin{array}{l}\text { Plastic } \\
\text { Response }\end{array}$ & $\begin{array}{l}\text { Elastic } \\
\text { Response }\end{array}$ & $\begin{array}{l}\text { Plastic } \\
\text { Response }\end{array}$ \\
\hline $\mathrm{L}_{\mathrm{b}}$ & $18.6 \%$ & $26.8 \%$ & $0.017 \%$ & $3.34 \%$ & $1.29 \%$ & $18.3 \%$ \\
\hline $\mathrm{L}_{\mathrm{b}} / 2$ & $15.2 \%$ & $33.5 \%$ & $0.015 \%$ & $3.01 \%$ & $1.21 \%$ & $17.3 \%$ \\
\hline $\mathrm{L}_{\mathrm{b}} / 4$ & $14.5 \%$ & $17.8 \%$ & $0.013 \%$ & $1.44 \%$ & $1.14 \%$ & $12.0 \%$ \\
\hline
\end{tabular}

\subsubsection{Beam Model Summary}

Overall, the analytical beam model has reasonable modelling capabilities. The elastic response model has an average percent difference of around $16 \%$, while the plastic model has an average error of around $26 \%$. In all three loading cases the percent difference between the models predicted transition and terminations loads, and the FEA estimated yield loads are under $15 \%$. In addition, from the three analyses, it can be observed that the energy predictions in the elastic response range are initially under-predicting, and transition to the plastic model early, compared to the FEA analyses. When comparing the plastic response of the analytical model to the FEA response as a whole, the model has an okay agreement. The main issue between the analytical and FEA models occur because the analytical model reaches center hinge collapse much earlier compared to the FEA collapse load. Both the elastic and plastic models' prediction error appears to improve as the patch load length decreased. In general, the model has an overall okay agreement, with a constant error which could be associated with the collapse load and energy, which has the potential to be corrected through the incorporation of an error correction term. 


\subsubsection{Plate}

To benchmark the plate meta-mode, FEA is used to examine four different patch load heights: $6.7 \mathrm{~cm}, 12.1 \mathrm{~cm}, 20.0 \mathrm{~cm}$, and $27.2 \mathrm{~cm}$. The same methods used to create the plate FEA models while creating the meta-model described in section 3.4.2.2 are adopted for the benchmarking analyses. New mesh convergence analyses are not conducted, but the trends from the first analyses are adapted with the new patch load heights. Both the 6.7 and 12.1 $\mathrm{cm}$ patch load responses are modelled with $5 \mathrm{~mm}$ edge length mesh elements, the $20 \mathrm{~cm}$ patch load is modelled with $3 \mathrm{~mm}$ long mesh edge lengths, and lastly, the $27.2 \mathrm{~cm}$ patch load is modelled with mesh elements with $2 \mathrm{~mm}$ long edges.

\subsubsection{Meta-Model Analysis}

Similar to the beam analyses, a quick visual assessment of the plate meta-models is conducted with Figure 4.7 to Figure 4.10, followed by the analyses of specific points from the individual elastic (Table 4.4) and plastic (Table 4.5) meta-models to more closely assess the individual models' prediction capabilities. In addition, the percent differences of both plate meta-models are also statistically analyzed (Table 4.8) to determine how consistent the percent difference is between the FEA and meta-models. Overall, from Figure 4.7 through Figure 4.10, the energy prediction of the plate meta-models has good agreement with the FEA models, but it can be observed that as the patch load height decreases, the difference between the models increases with the increase of the contact forces. 


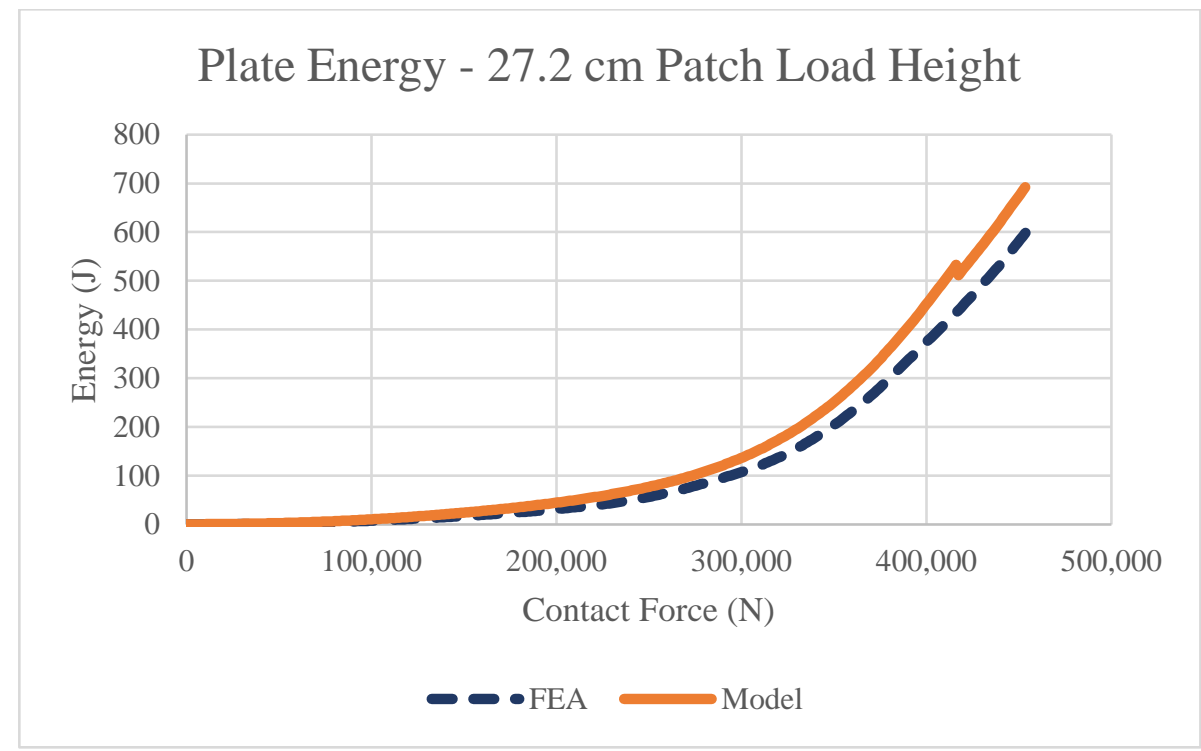

Figure 4.7 Plate Energy Analysis - $27.2 \mathrm{~cm}$ Patch Load Height

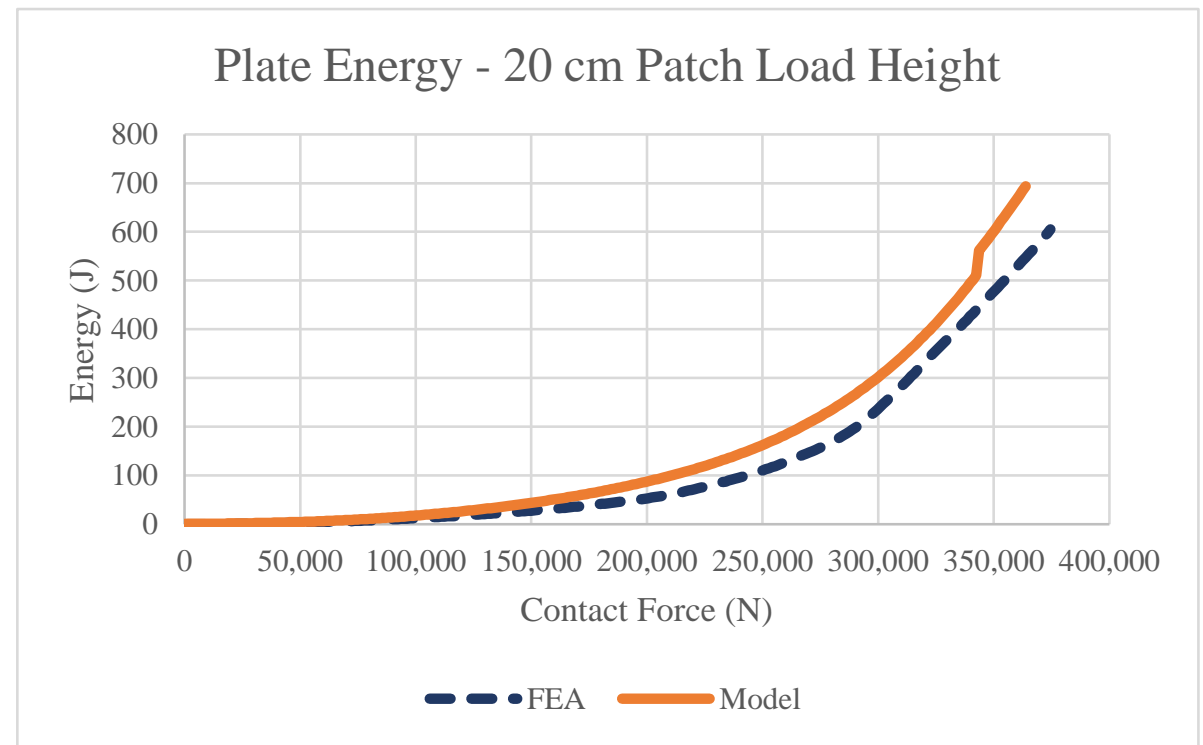

Figure 4.8 Plate Energy Analysis - $20 \mathrm{~cm}$ Patch Load Height 


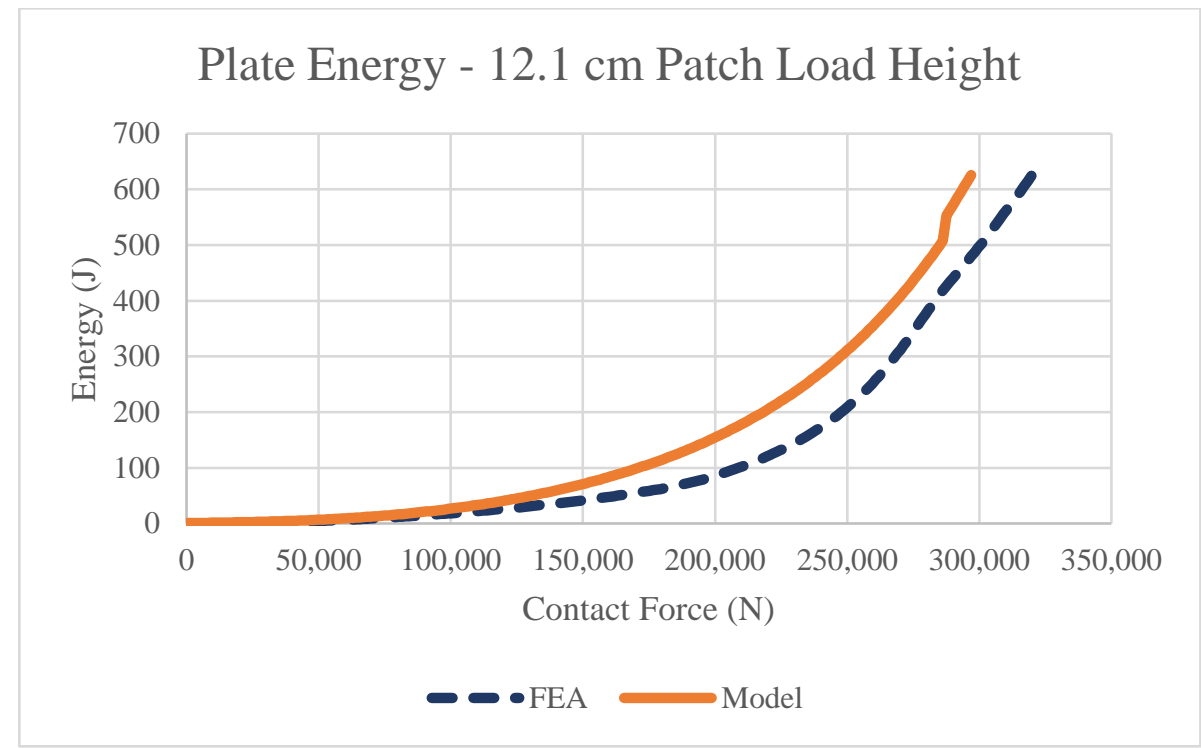

Figure 4.9 Plate Energy Analysis - $12.1 \mathrm{~cm}$ Patch Load Height

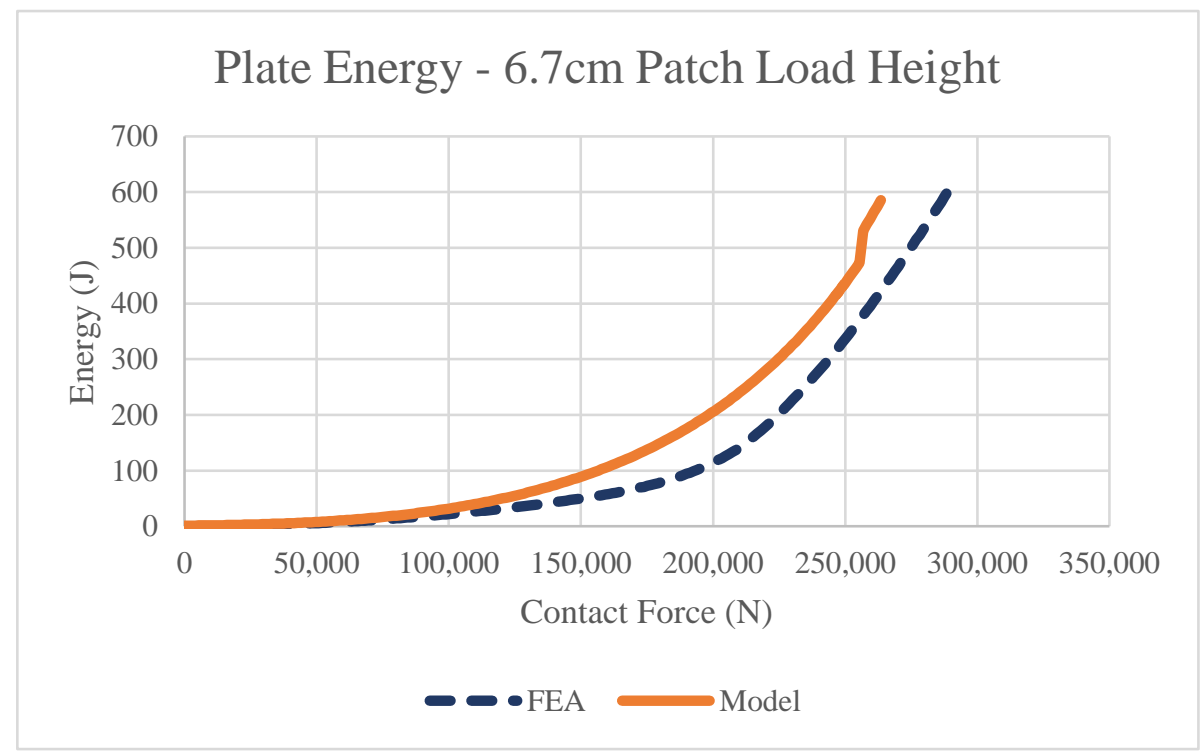

Figure 4.10 Plate Energy Analysis - $6.7 \mathrm{~cm}$ Patch Load Height

First, the elastic plate meta-model is compared to the FEA analyses of the four different patch load heights, Table 4.4. For each patch load, three different contact forces are analyzed within the model's estimated transition load: a relatively small load, a mid-range load, a contact force close to the transition load. For all four patch load heights, the smallest 
load has the worst percent difference compared to the FEA internal energy response. The low contact forces, safely within a harmless material response, are the least concerning load to have a high percent error. Even if the model has poor energy prediction in the low energy elastic range, there is a large buffer before any concerning loads occur. Otherwise, the percent difference does not exceed $15 \%$ and the prediction capabilities of the model increase with the increase in the patch load height.

Table 4.4 Elastic Plate FEA Verification Points

\begin{tabular}{|c|c|c|c|c|c|}
\hline \multicolumn{3}{|c|}{ Verification Point } & \multirow[b]{2}{*}{$\begin{array}{l}\text { FEA Internal } \\
\text { Energy }(\mathrm{J})\end{array}$} & \multirow[b]{2}{*}{$\begin{array}{l}\text { Model Predicted } \\
\text { Internal Energy }(\mathrm{J})\end{array}$} & \multirow[b]{2}{*}{$\begin{array}{l}\text { Percent } \\
\text { Difference }\end{array}$} \\
\hline & $\begin{array}{l}\text { Contact } \\
\text { Force }(\mathrm{N})\end{array}$ & $\begin{array}{l}\text { Patch Load } \\
\text { Height }(\mathrm{cm})\end{array}$ & & & \\
\hline 1 & 49,493 & 6.7 & 5.37 & 3.27 & $39.1 \%$ \\
\hline 2 & 148,923 & 6.7 & 48.7 & 56.3 & $15.5 \%$ \\
\hline 3 & 273,952 & 6.7 & 495 & 445 & $10.2 \%$ \\
\hline 4 & 49,487 & 12.1 & 4.51 & 2.84 & $36.9 \%$ \\
\hline 5 & 148,192 & 12.1 & 40.7 & 45.4 & $11.6 \%$ \\
\hline 6 & 273,915 & 12.1 & 338 & 316 & $6.61 \%$ \\
\hline 7 & 102,712 & 20.0 & 13.2 & 11.1 & $15.9 \%$ \\
\hline 8 & 286,141 & 20.0 & 186 & 192 & $3.08 \%$ \\
\hline 9 & 359,513 & 20.0 & 527 & 477 & $9.51 \%$ \\
\hline 10 & 75,139 & 27.2 & 4.36 & 3.12 & $28.5 \%$ \\
\hline 11 & 279,254 & 27.2 & 84.1 & 83.1 & $1.20 \%$ \\
\hline 12 & 419,951 & 27.2 & 450 & 439 & $2.57 \%$ \\
\hline
\end{tabular}

Similarly, the FEA analyses of the plastic plate meta-models examined three different contact forces within the meta-models' predicted transition and termination loads for the four different patch load heights. Overall, the plastic response models have great agreement with the FEA responses (Table 4.5). Opposite to the elastic model, the plastic energy metamodel has a slight increase in percent difference with the increase in patch load height but never exceeds an error of more than $4 \%$. 
Table 4.5 Plastic Plate FEA Verification Points

\begin{tabular}{|c|c|c|c|c|c|}
\hline \multicolumn{3}{|c|}{ Verification Point } & \multirow[b]{2}{*}{$\begin{array}{l}\text { FEA Internal } \\
\text { Energy }(\mathrm{J})\end{array}$} & \multirow[b]{2}{*}{$\begin{array}{l}\text { Model Predicted } \\
\text { Internal Energy }(\mathrm{J})\end{array}$} & \multirow[b]{2}{*}{$\begin{array}{l}\text { Percent } \\
\text { Difference }\end{array}$} \\
\hline & $\begin{array}{l}\text { Contact } \\
\text { Force }(\mathrm{N})\end{array}$ & $\begin{array}{l}\text { Patch Load } \\
\text { Height }(\mathrm{cm})\end{array}$ & & & \\
\hline 1 & 280,908 & 6.7 & 544 & 542 & $0.45 \%$ \\
\hline 2 & 281,846 & 6.7 & 553 & 549 & $0.70 \%$ \\
\hline 3 & 283,583 & 6.7 & 562 & 563 & $0.22 \%$ \\
\hline 4 & 311,545 & 12.1 & 572 & 563 & $1.66 \%$ \\
\hline 5 & 312,969 & 12.1 & 579 & 573 & $0.99 \%$ \\
\hline 6 & 314,039 & 12.1 & 588 & 582 & $1.11 \%$ \\
\hline 7 & 370,151 & 20.0 & 583 & 585 & $0.35 \%$ \\
\hline 8 & 378,164 & 20.0 & 628 & 637 & $1.41 \%$ \\
\hline 9 & 383,566 & 20.0 & 661 & 673 & $1.85 \%$ \\
\hline 10 & 449,375 & 27.2 & 578 & 556 & $3.85 \%$ \\
\hline 11 & 462,584 & 27.2 & 642 & 621 & $3.25 \%$ \\
\hline 12 & 469,168 & 27.2 & 676 & 655 & $3.08 \%$ \\
\hline
\end{tabular}

The models' transition and termination loads are compared to the full through-thickness yield of the FEA responses, Table 4.6. In all four cases, the predicted loads have great agreement with the highest percent difference of only 5\%. For the smaller patch load heights, the model appears to under-predict the transition and termination loads slightly, but slightly over-predicts the loads of the larger patch load heights.

Table 4.6 Plate Model Transition

\begin{tabular}{|r|r|l|l|l|r|l|r|}
\hline \multicolumn{2}{|c|}{ Verification Point } & \multicolumn{2}{c|}{ FEA } & \multicolumn{4}{c|}{ Model } \\
\hline & & $\begin{array}{l}\text { Center- } \\
\text { Initial } \\
\text { hinge } \\
\text { Yatch Load } \\
\text { Yield } \\
\text { Collapse } \\
(\mathrm{N})\end{array}$ & $\begin{array}{l}\text { Initial } \\
\text { Yield } \\
(\mathrm{N})\end{array}$ & $\begin{array}{l}\text { Penter- } \\
\text { Pinge } \\
\text { Difference }\end{array}$ & $\begin{array}{l}\text { Collapse } \\
(\mathrm{N})\end{array}$ & $\begin{array}{l}\text { Percent } \\
\text { Difference }\end{array}$ \\
\hline 1 & 6.7 & 283,583 & 288,934 & 279,460 & $1.45 \%$ & 284,952 & $1.38 \%$ \\
\hline 2 & 12.1 & 315,644 & 320,994 & 309,077 & $2.08 \%$ & 318,617 & $0.74 \%$ \\
\hline 3 & 20.0 & 358,423 & 374,472 & 365,789 & $2.06 \%$ & 385,193 & $2.86 \%$ \\
\hline 4 & 27.2 & 417,276 & 454,724 & 439,245 & $5.26 \%$ & 475,804 & $4.64 \%$ \\
\hline
\end{tabular}


In addition to analyzing the estimated termination loads, the response energy at both the model and FEA termination points are compared to see if the different termination loads affect the estimated maximum energy capacity of the plate (Table 4.7). The smaller patch load heights have smaller percent differences between the predicted maximum energy capacities, which also agrees with the smaller percent differences of the predicted terminations loads. The larger patch load heights appear to be over-predicting the maximum capacity of the plate, by about $15 \%$ at the termination limit state of 3-hinge collapse, again following the same trends as the model's predicted termination load.

Table 4.7 Plate Termination Energy Prediction

\begin{tabular}{|c|c|c|c|c|}
\hline \multicolumn{2}{|r|}{ Verification Point } & \multirow{2}{*}{$\begin{array}{l}\text { FEA } \\
\text { Energy }(\mathrm{J})\end{array}$} & \multirow{2}{*}{$\begin{array}{l}\text { Model } \\
\text { Energy }(\mathrm{J})\end{array}$} & \multirow{2}{*}{$\begin{array}{l}\text { Percent } \\
\text { Difference }\end{array}$} \\
\hline & Patch Load Height $(\mathrm{cm})$ & & & \\
\hline 1 & 6.7 & 602 & 585 & $2.75 \%$ \\
\hline 2 & 12.1 & 633 & 626 & $1.17 \%$ \\
\hline 3 & 20.0 & 605 & 693 & $14.6 \%$ \\
\hline 4 & 27.2 & 604 & 692 & $14.6 \%$ \\
\hline
\end{tabular}

When the percent differences of the full elastic energy response, dependent on the contact force, are analyzed, the energy response does have a higher variance and standard deviation, but the average error, of all the different patch load heights, is around $15 \%$. The large variance and standard deviations are mainly due to the larger prediction errors associated with the smaller contact forces observed in Table 4.4. The statistical analysis of the plastic energy meta-model found that the model has great agreement with the FEA responses. The percent difference between the FEA and plastic energy model, dependent on the contact force, overall has a very low average error of $1.5 \%$, in addition to a low variance and standard deviation. It is interesting to note that as the patch load height decreases the percent difference of the elastic model increases, but the percent difference 
of the plastic model decreases with the decrease of the patch load height. This trend is likely due to the decrease in the plastic response range with the decrease in the patch load height, which in turn reduces the plastic meta-model response surface and increases the accuracy of the model.

Table 4.8 Plate Meta-Model Energy Prediction Statistical Analyses

\begin{tabular}{|c|c|c|c|c|c|c|}
\hline \multirow{2}{*}{$\begin{array}{l}\text { Patch } \\
\text { Load } \\
\text { Height } \\
(\mathrm{cm})\end{array}$} & \multicolumn{2}{|c|}{ Mean } & \multicolumn{2}{|c|}{ Variance } & \multicolumn{2}{|c|}{ Standard Deviation } \\
\hline & $\begin{array}{l}\text { Elastic } \\
\text { Response }\end{array}$ & $\begin{array}{l}\text { Plastic } \\
\text { Response }\end{array}$ & $\begin{array}{l}\text { Elastic } \\
\text { Response }\end{array}$ & $\begin{array}{l}\text { Plastic } \\
\text { Response }\end{array}$ & $\begin{array}{l}\text { Elastic } \\
\text { Response }\end{array}$ & $\begin{array}{l}\text { Plastic } \\
\text { Response }\end{array}$ \\
\hline 27.2 & $10.1 \%$ & $3.46 \%$ & $0.928 \%$ & $0.0027 \%$ & $9.64 \%$ & $0.516 \%$ \\
\hline 20.0 & $14.9 \%$ & $1.39 \%$ & $0.792 \%$ & $0.0052 \%$ & $8.90 \%$ & $0.721 \%$ \\
\hline 12.1 & $18.5 \%$ & $0.740 \%$ & $1.11 \%$ & $0.0033 \%$ & $10.6 \%$ & $0.578 \%$ \\
\hline 6.7 & $19.1 \%$ & $0.346 \%$ & $1.35 \%$ & $0.0003 \%$ & $11.6 \%$ & $0.167 \%$ \\
\hline
\end{tabular}

\subsubsection{Plate Model Summary}

Overall, the plate models have good agreement with the FEA analyses, with the average percent differences around $8.5 \%$. The plastic energy response model has very good agreement, which is the more critical model that predicts the energy response of the plate during the more significant loading events. The elastic energy response has a higher average percent difference, but the poorest performing region of the model is in the very safe elastic response region, while the rest of the elastic model has reasonably good agreement.

\subsection{FEA Grillage Analyses}

The assembled algorithm is also benchmarked against a grillage structure, to identify to what degree the evaluation of the isolated structural components within the algorithm affects the prediction of the full structural energy response. The FEA grillage structure is 
also implemented to investigate the two main impact locations, an impact directly on a frame, and an impact centred between two frames. Further, the algorithm is compared to two different loading conditions. First, the algorithm is compared to the model's assumed loading condition of a rectangular patch load. Then, the algorithm is analyzed against a more realistic impact due to an ice wedge interaction.

The same grillage structure is used to benchmark both loading conditions, with the addition of a patch load part, for the patch load loading condition (Figure 4.11). A separate patch load part is added within the plate to ensure consistent size and location of the patch load. Two different grillage structures are used for the different impact location investigations, with the only difference being the arrangement of the frames. The grillage structures are arranged so that either a frame (Figure 4.11) or frame spacing (Figure 4.12) is centred on the plate. In addition, to evaluate the capability of the beam model modelling other crosssections, the grillage structure is created with angle bar frames, instead of the model's assumed flat bar.

For the grillage analyses, a similar setup to the previous plate FEA analyses is implemented. An explicit solver is employed for both the patch load and ice impact loading events. The grillage model adopted the same bi-linear material model (Figure 3.10) assumed while creating the plate model, and to analyze the beam model against a more realistic material response. The material model assumes structural steel with a density of $7850 \mathrm{~kg} / \mathrm{m}^{3}$, Young's modulus of $200 \mathrm{GPa}$, a Poisson's ratio of 0.3 , a yield strength of $250 \mathrm{MPa}$, and an $E_{T a n}$ of $1500 \mathrm{MPa}$. A large grillage area is assumed (3.6 x $\left.2.1 \mathrm{~m}\right)$ with fixed nodal boundary 
conditions (Figure 4.12) to remove any possible boundary effects while keeping the structure fixed in place.

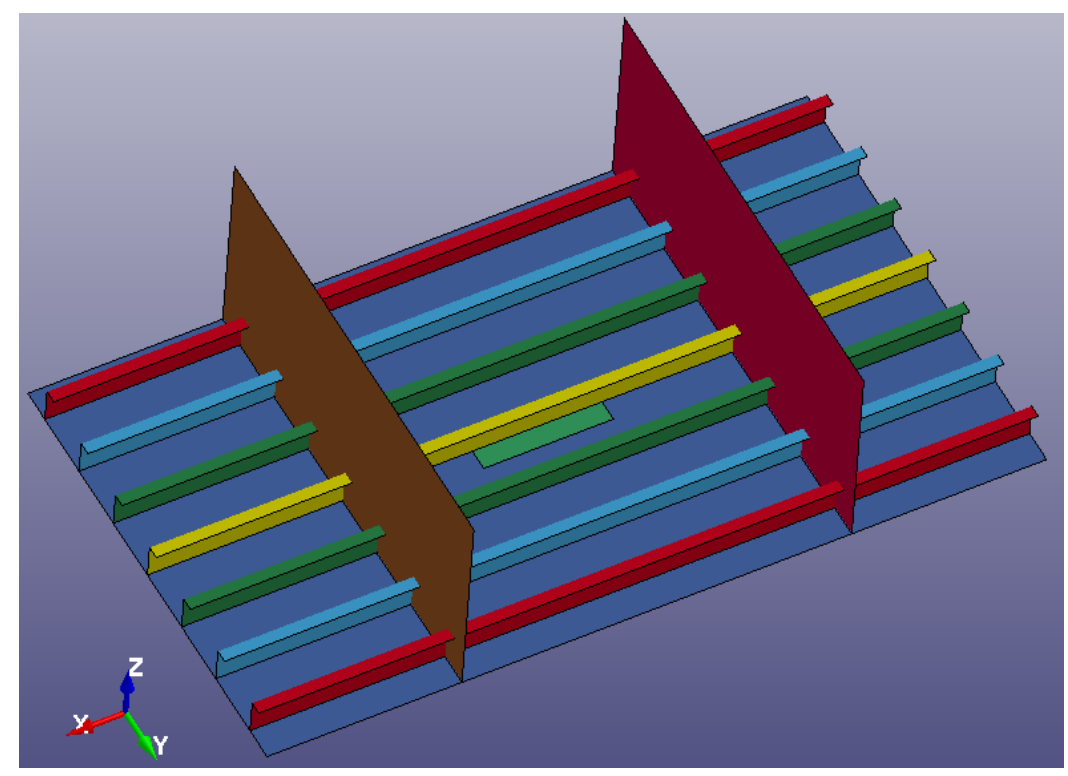

Figure 4.11 Grillage Structure - Patch Load on Frame

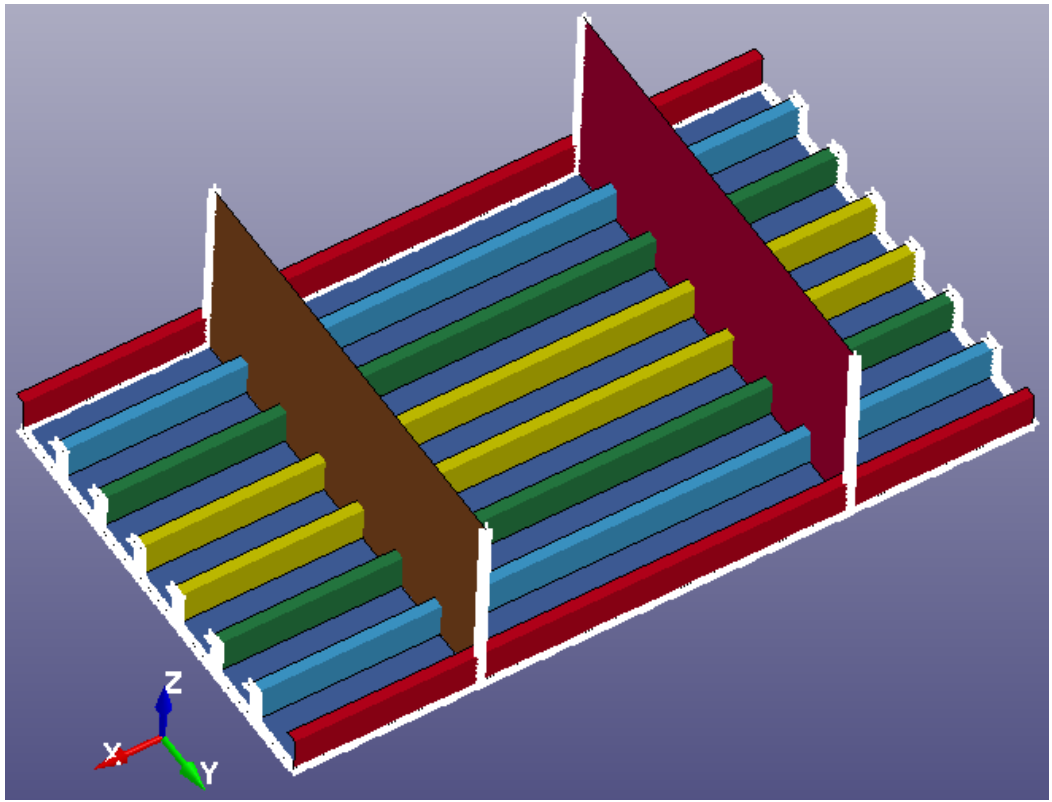

Figure 4.12 Grillage Structure - Loading Condition Between Frames with Fixed Boundary Conditions 
A generic grillage structure is displayed with the dimensions, in millimetres, shown in Figure 4.13 and Figure 4.14, for a patch load on the center of a frame. For the loading case of an ice piece impact, the patch is removed, and the plate is modelled as one continuous part. For the grillage structure impact between frames, the frames are shifted vertically by $0.150 \mathrm{~m}$, with frames on both the top and bottom edges of the plate. The plate and patch surfaces are modelled with a $12 \mathrm{~mm}$ thickness, with the reference surface at the top of the surfaces. The frame surfaces are modelled with a $27.5 \mathrm{~mm}$ through-thickness and the reference surface on the bottom of the surface. The girder surfaces are modelled with an 8 mm through-thickness, with a centred reference surface. The whole grillage is modelled with shell elements, with a shear factor of $5 / 6$ and five through-thickness integration points.

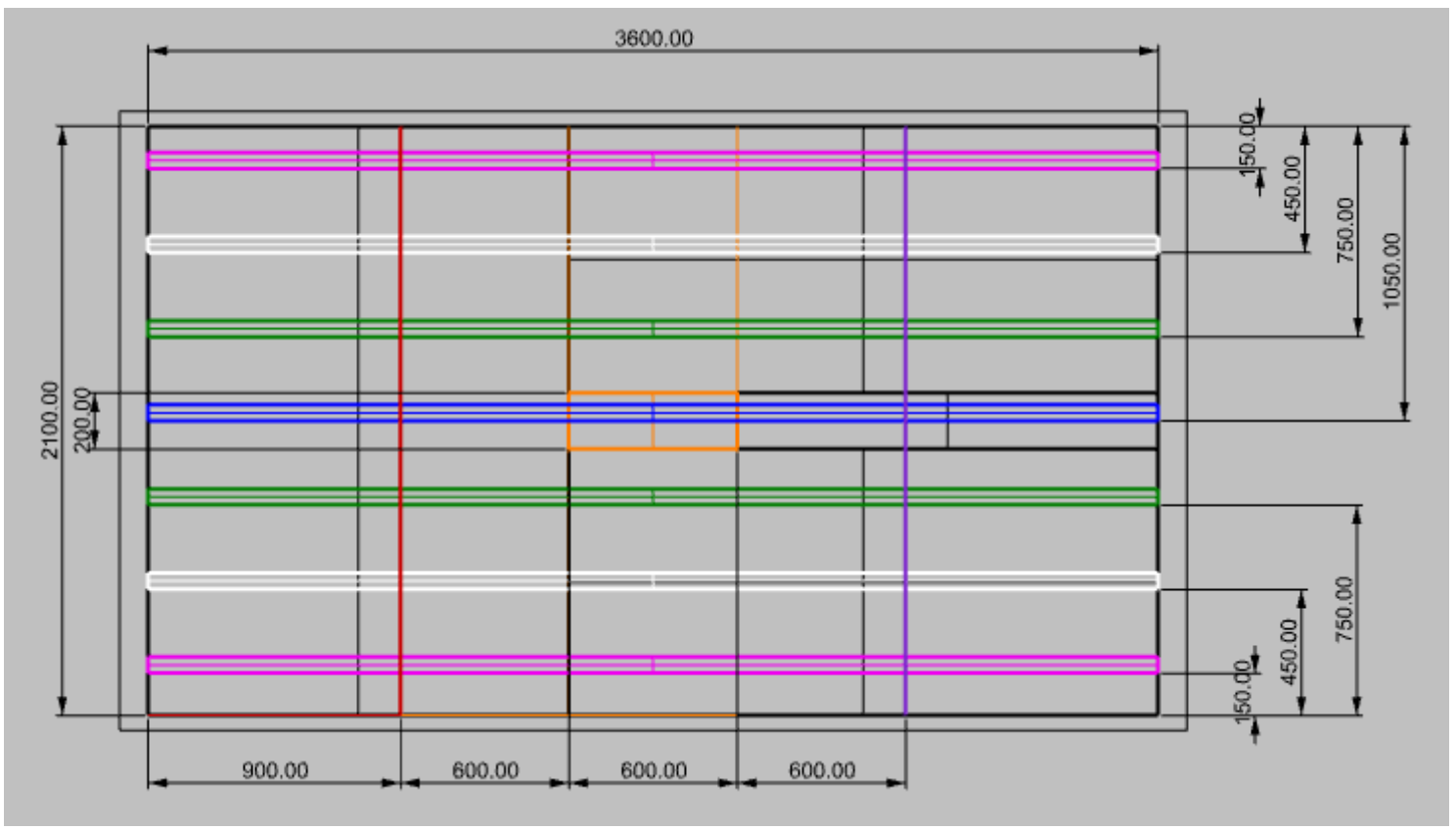

Figure 4.13 Grillage Structure - Top View Dimensions (In mm) 


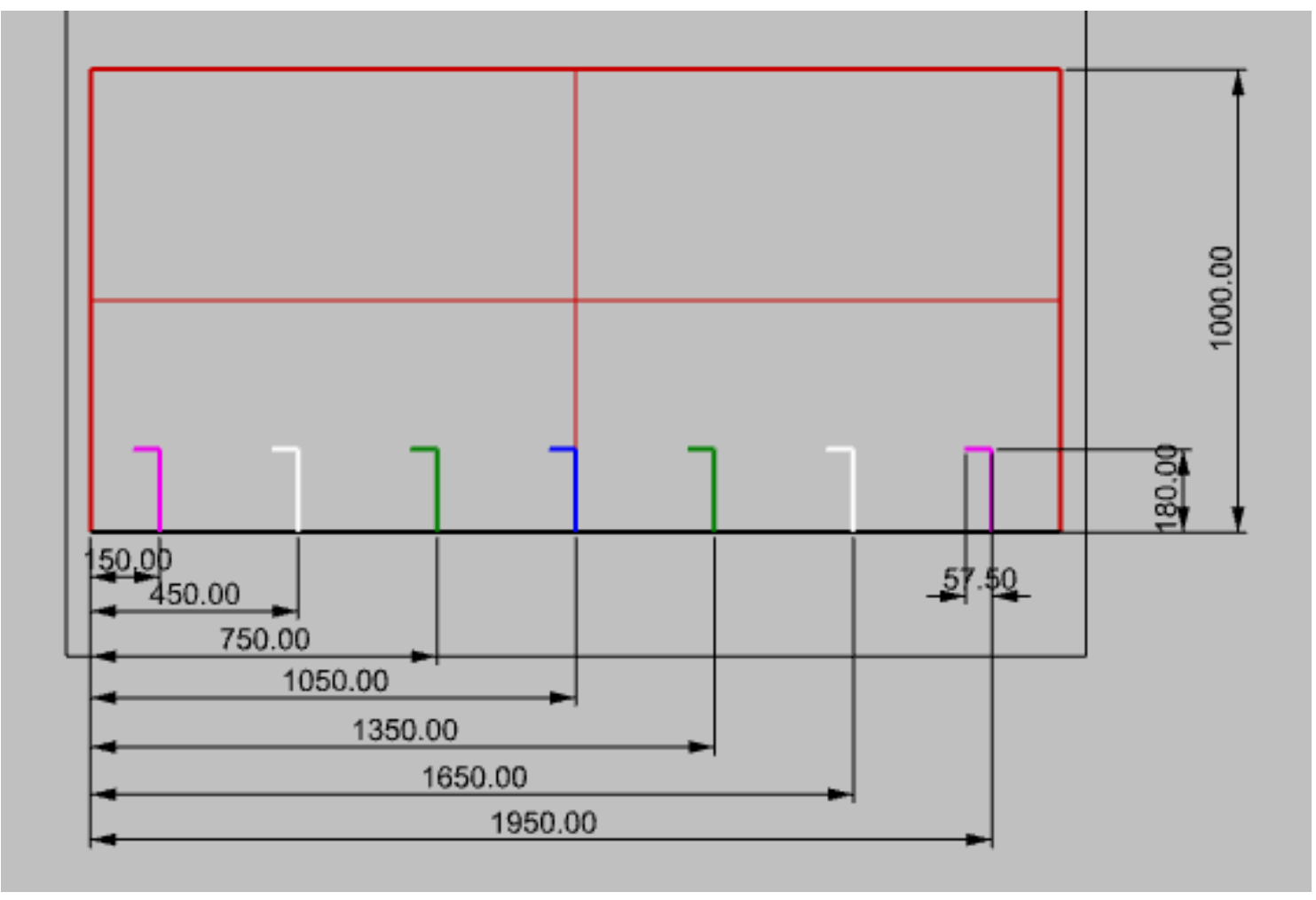

Figure 4.14 Grillage Structure - Left Side View Dimensions (In mm)

\subsubsection{Patch Load}

An FEA model is created assuming a rectangular patch load contact area $(0.6 \mathrm{~m}$ by $0.2 \mathrm{~m})$ to maintain the contact geometry assumed while creating the algorithm, to focus on the evaluation of the model's ability to estimate the structural response of a grillage structure. The only other addition to this FEA analysis is the inclusions of the more realistic bi-linear material model, which incorporates a linear plastic response. The patch load analyses examined the algorithm's response up to the 3-hinge collapse limit state, for the two loading scenarios: an impact directly on the frame (Figure 4.11), and an impact centred between two frames (Figure 4.12). For the patch load loading condition, a uniform pressure is applied with a unit ramp (Figure 3.11b) to a set of shell elements established by the patch load part. The pressure is increased through a unit ramp over a 1 second time interval until 
the maximum pressure of $5 \mathrm{MPa}$ is reached, to create a static loading event and remove and inertial effects from the response.

As two different grillage structures are being analyzed, two different mesh convergence analyses are conducted (Figure 4.15). In both cases, there is not a huge change in the maximum deflection responses as the element size decrease, with $13 \mathrm{~mm}$ sized elements chosen for both grillage structures. For the patch load on the frame analysis, $13 \mathrm{~mm}$ is chosen since even though there is a slight spike in the displacement response as it also appears to be stabilizing out towards $0.0058 \mathrm{~m}$. For the analysis of the patch load between the frames, $13 \mathrm{~mm}$ is chosen since the change in the response between the elements smaller than $13 \mathrm{~mm}$ is minimal.

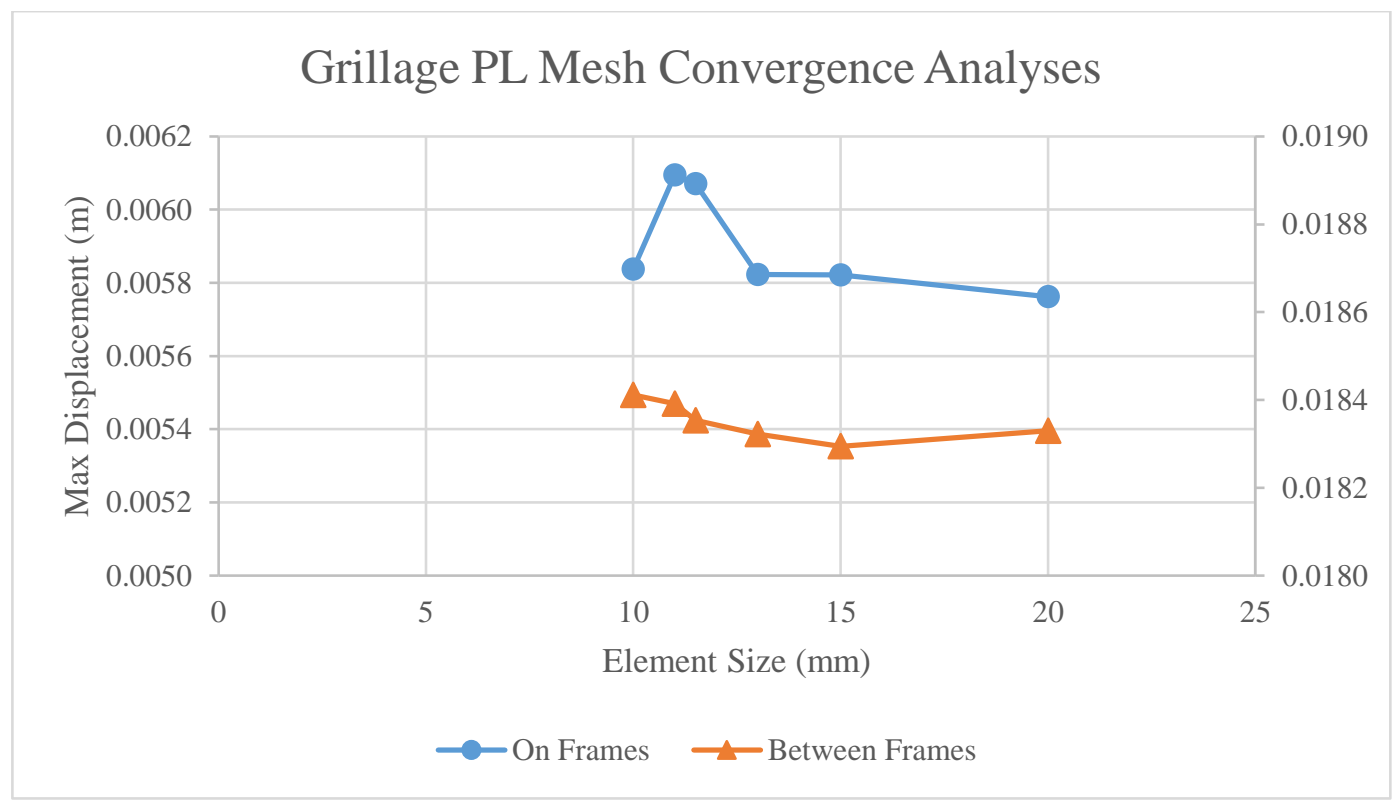

Figure 4.15 Grillage Structure Patch Load - Mesh Convergence Analyses 


\subsubsection{Grillage Patch Load Analysis}

For this analysis, the individual internal energy responses of the structural elements, in addition to the complete grillage response of the FEA models, are examined. First, the frame response is assessed. In Figure 4.16 the algorithm's analytical strain energy is compared to the FEA internal energy responses of the centre frame for the impact centred on a frame, and the two frames on either side of the impact for a loading event between two frames.

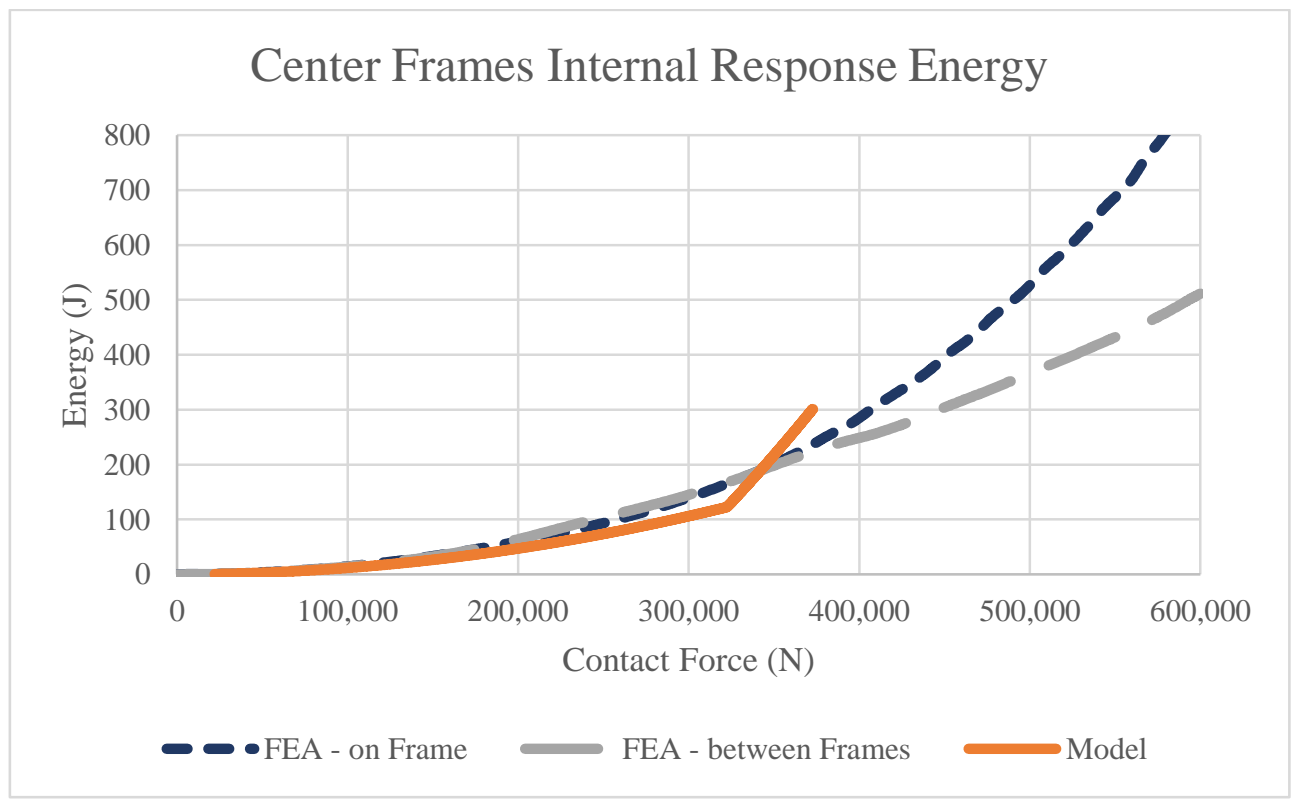

Figure 4.16 Grillage Patch Load - Frame Energy Response

Examining the energy responses of the beam in the figure above, the energy responses of both the FEA analyses are similar, with the algorithm response slightly lower than the FEA estimations. As the elastic response continues the energy response starts to drop, but once the model transitions into the plastic response, the algorithm starts to approach the FEA models again. In addition, even with the alternate cross-section of the frame and the more 
realistic boundary condition of the surrounding grillage structure, the beam response still follows the same trends observed made while analyzing the structural model in isolation.

Next, the plate response of the grillages and model, with a rectangular patch load, are examined as Figure 4.17. Since in the FEA the patch load is established as a separate part, to help keep a consistent patch load size, the full energy response of the plate is represented by the sum of both the plate and the patch load grillage parts. Figure 4.17 compares the algorithm's predicted plate response to the energy response of just the patch load and full plate response of the plate and patch load parts.

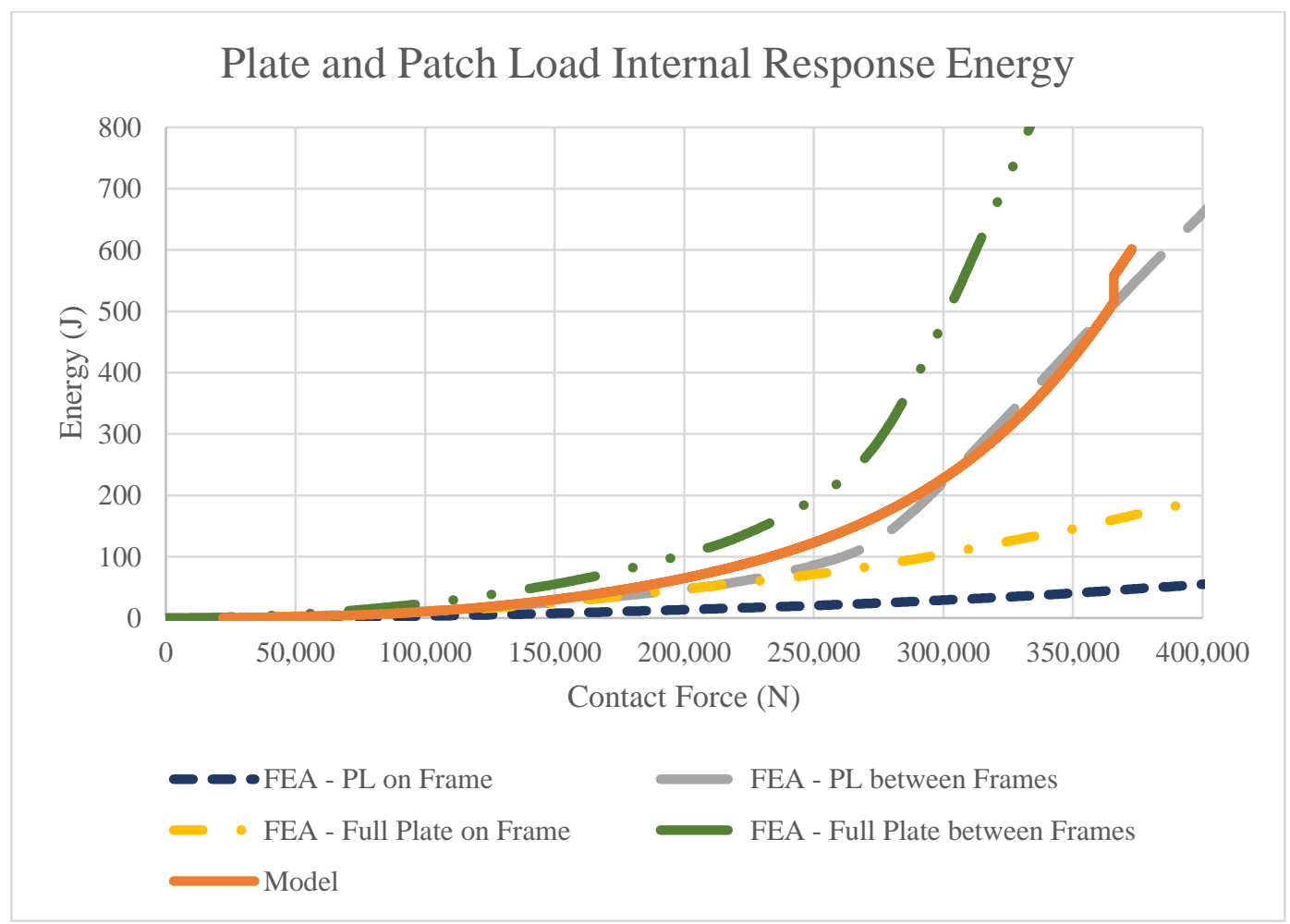

Figure 4.17 Grillage Patch Load - Plate Energy Response

It can be observed that the FEA response for the patch load between the frames has a very similar response to the algorithm's plate meta-model, which most closely corresponds to 
the design scenario that the algorithm's meta-model is created with. But when the full plate response for the large grillage structure for the patch load between the frames is taken into consideration, the algorithm now under-predicts the energy response. In contrast, both the patch load and full plate responses for the loading condition on the frame, are substantially less than the model's prediction. Since with an impact on the frame the frame alleviates some of the load which is not taken into account by the plate model.

Examining the FEA full grillage plate responses (Figure 4.17) finds that the algorithm's response falls in between the two different loading events. The full plate response of the patch load between the frames has a lot more capacity for energy absorption and deflection compared to the smaller plate, which is assumed to create the meta-model. In contrast, the FEA plate response, for the loading event of a patch load directly on the frame, absorbs notably less energy compared to the predicted algorithm response. This difference between the FEA model of the patch load on the frame and the algorithm's plate model is due to the substantial amount of the plate response supported by the frame, which is not accounted for in the plate's meta-model design scenario. Therefore, in practice, the plate meta-model does not favour either loading event but predicts a neutral response in between the two.

With the individual structural elements of the FEA models assessed with more realistic boundary conditions and compared to the individual structural models, now the full energy response of the grillage is compared to the full structural energy prediction of the algorithm. Examining Figure 4.18, at first glance, it looks like the algorithm has a decent agreement with the FEA analyses. The loading event of the patch load on the frame appears to have great agreement for the majority of the interaction, until about $300 \mathrm{kN}$ when the algorithm 
starts to have an increased energy response compared to the FEA analysis. This difference between the algorithm and the on the frame loading event is likely due to the overprediction of the plate response of the plate meta-model. The algorithm notably underpredicts the loading event between frames, which is likely due to the substantial underprediction of the plate response.

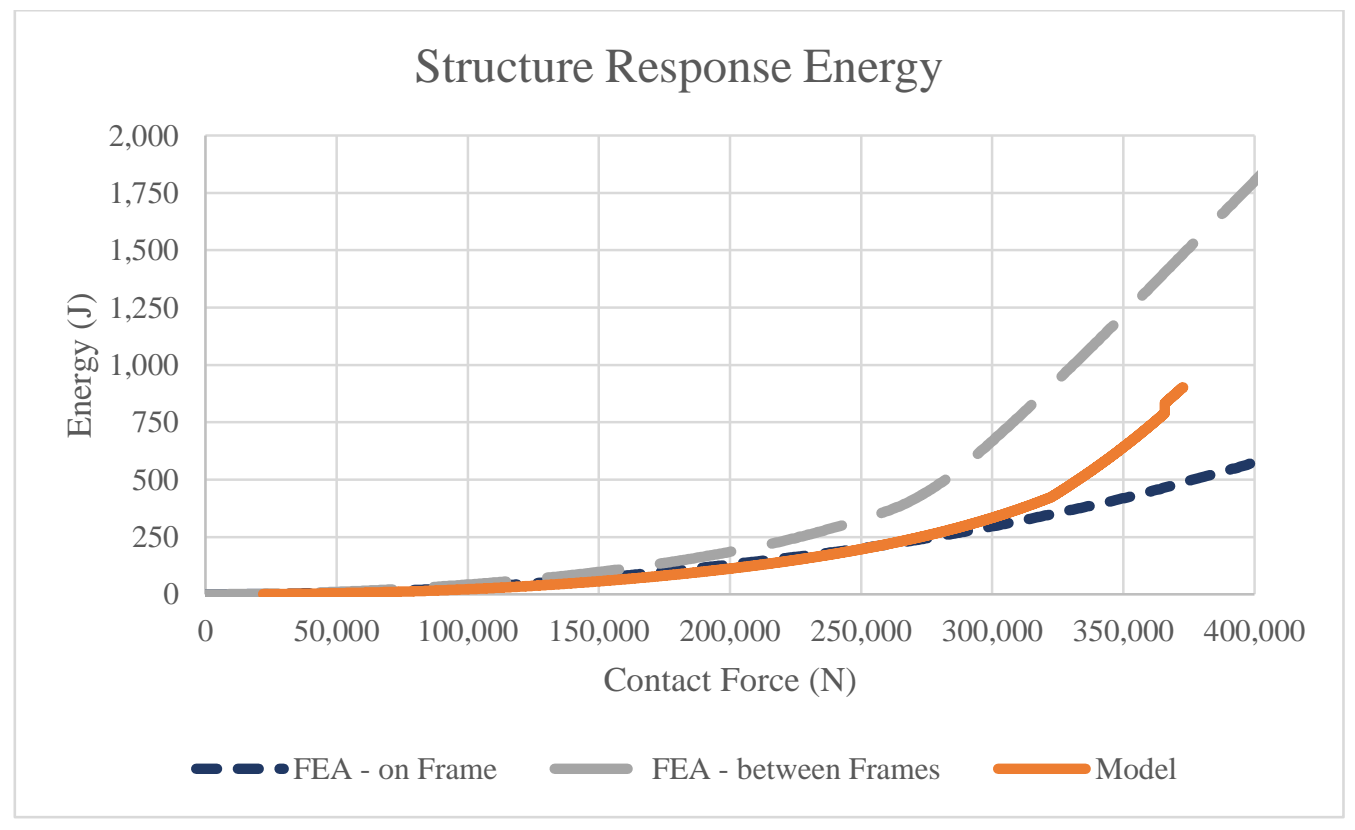

Figure 4.18 Grillage Patch Load - Full Grillage Energy Response

In addition to the visual analyses, the algorithm is also evaluated statistically against the FEA models. First, in Table 4.9 the estimated yield loads are compared. It is interesting to observe that depending on the loading event only one of the structural components yields, compared to the algorithm, which assumes that both structural elements are loaded to their full capacity. It should also be noted that with the grillage FEA analyses the structural components do not yield as uniformly as the individual analytical models assume, so the grillage yield points are estimated based on the first occurrence of through-thickness yield to a notably concentrated area of yielded elements. 
For the loading scenario of the patch load centred on the frame, only the beam yielded, as it is carrying a more direct portion of the load compared to the plate. Comparing the contact forces for the patch load on the frame to cause yield, to the transition and termination loads estimated with the beam model, it can be observed that the frame within the grillage structure has a much higher capacity with an average increased load of $30 \%$ before the beam starts to yield and fully collapses. The plate transition and termination loads follow those estimated by the FEA loading event between the frames very closely, which is likely due to the algorithm's assumed plate loading scenario, following closer to the FEA loading event compared to the beam model's design scenario.

Table 4.9 Grillage Patch Load - Load at Yield

\begin{tabular}{|c|c|c|c|c|}
\hline \multirow{2}{*}{\multicolumn{2}{|c|}{$\frac{\text { Model Yield Load }}{\text { Beam }}$}} & \multicolumn{3}{|c|}{ FEA Yield Load } \\
\hline & & \multicolumn{2}{|c|}{ On Frames - Beam } & Percent Difference \\
\hline Edge & 322,463 & Initial & 450,000 & $28.3 \%$ \\
\hline Center & 372,624 & Full & 579,000 & $35.6 \%$ \\
\hline \multicolumn{2}{|c|}{ Plate } & \multicolumn{2}{|c|}{ Between Frames - Plate } & Percent Difference \\
\hline Edge & 365,790 & Initial & 375,000 & $2.46 \%$ \\
\hline Center & 385,194 & Full & 402,000 & $4.18 \%$ \\
\hline
\end{tabular}

Similar to the individual structural elements analyses, a statistical analysis comparing the predicted energy depending on the contact force of the whole loading event is conducted for the individual components and the full structural response, found in Table 4.10. The mean percent difference for both loading events for the beam is very similar, around $20 \%$, which follows what is observed in Figure 4.16. The plate response in both cases has a very high percent difference. The plate energy response for a patch load on the frame has a $73 \%$ difference and a very high variance. The loading event of a patch load between the frames has a better and more consistent percent difference, likely due to the design scenario being 
more akin to the loading event the grillage is experiencing. Even with the very high percent difference of the plate response, the total error of the full grillage response is reasonable. The loading event of the patch load on the frame has an error of around $20 \%$ likely due to the overestimation of the plate response compensating for the underestimation of the frame response. The patch load between the frames is much higher, around 50\%, but the percent difference is more consistent, which has the potential to be corrected with a correction factor.

Table 4.10 Grillage Patch Load Energy Prediction Statistical Analysis

\begin{tabular}{|r|l|r|r|r|}
\hline & $\begin{array}{l}\text { Patch Load } \\
\text { Frame } \\
\text { Location }\end{array}$ & Mean Error & Variance Error & $\begin{array}{l}\text { Standard } \\
\text { Deviation Error }\end{array}$ \\
\hline \multirow{3}{*}{ Beam } & On & $19.9 \%$ & $245 \%$ & $15.6 \%$ \\
\cline { 2 - 5 } Plate & Between & $21.7 \%$ & $224 \%$ & $15.0 \%$ \\
\hline \multirow{3}{*}{ Total } & On & $73.0 \%$ & $5619 \%$ & $75.0 \%$ \\
\cline { 2 - 5 } & Between & $49.6 \%$ & $91.0 \%$ & $9.54 \%$ \\
\hline & On & $21.5 \%$ & $209 \%$ & $14.4 \%$ \\
\hline
\end{tabular}

To help visualize the percent difference discussed in Table 4.10, a box and whisker plot is created as Figure 4.19. The response does surpass a $120 \%$ difference, but the figure is capped at $120 \%$ since the essential information is captured. Both structural components and the full structural response are plotted for both the loading scenarios of the patch load on or centred between frames. 


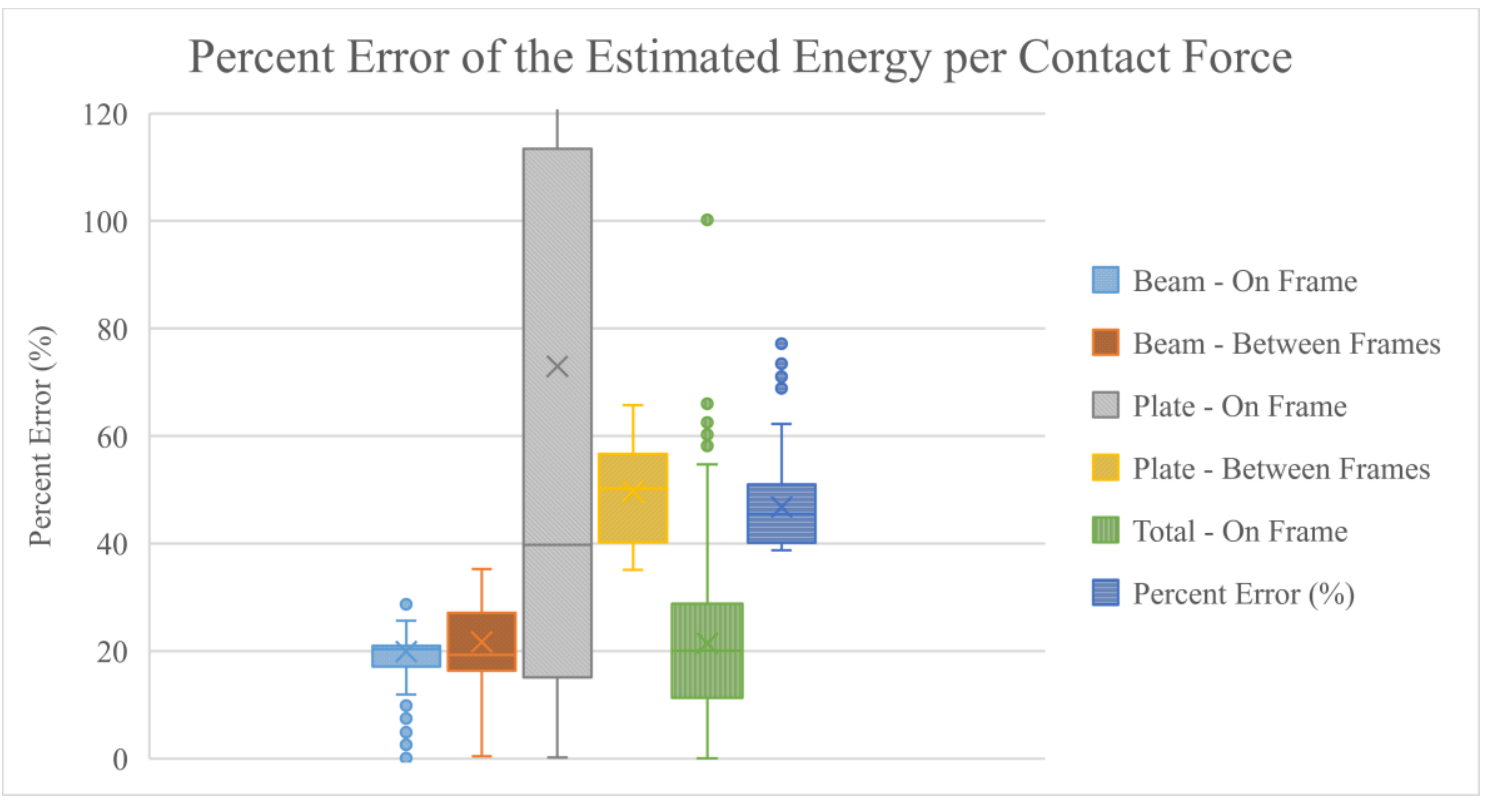

Figure 4.19 Grillage Patch Load - Energy Prediction Box and Whisker Plot

\subsubsection{Grillage Patch Load Summary}

Overall, the model has reasonable agreement to a grillage loaded with a patch load centred on a frame, which has an average error of around 20\%. From Figure 4.18 for a patch load on the frame, the algorithm is overestimating how much energy the grillage is absorbing during the plastic response, which could be an issue if it is not properly accounted for, since that would falsely increase the algorithm's estimated contact force. For the loading event of a patch load between frames, the percent difference increases to about $50 \%$, but the energy response has a more consistent error, which could be corrected through an error term. For the patch load between frames though, the algorithm is underestimating the energy absorbed by the grillage especially in the plastic response range, which reduces the maximum contact force since the structure is absorbing less of the impact energy, creating a more conservative response. The decision to assess the structural elements separately 
appears more in the errors associated with the plate model, but overall, the structural response of the algorithm compared to both FEA grillage models has an okay agreement.

\subsubsection{Ice Body Interaction}

The last benchmarking analysis conducted examines the effects of more realistic contact geometry, produced by a $0.49 \mathrm{~m}$ thick ice-wedge $\left(135^{\circ}\right)$ and angled grillage structure (rotated $55^{\circ}$ about the $\mathrm{x}$-axis). The grillage has a similar set-up to the FEA patch load models, except for the loading condition. An ice wedge is added to the interaction and is set with an initial velocity, which produces a kinetic interaction. For this FE model, Figure 4.20, the contact is set as automatic surface to surface, with both the ice parts set as the slave surfaces and the plate and center frame parts modelled as the master surfaces.

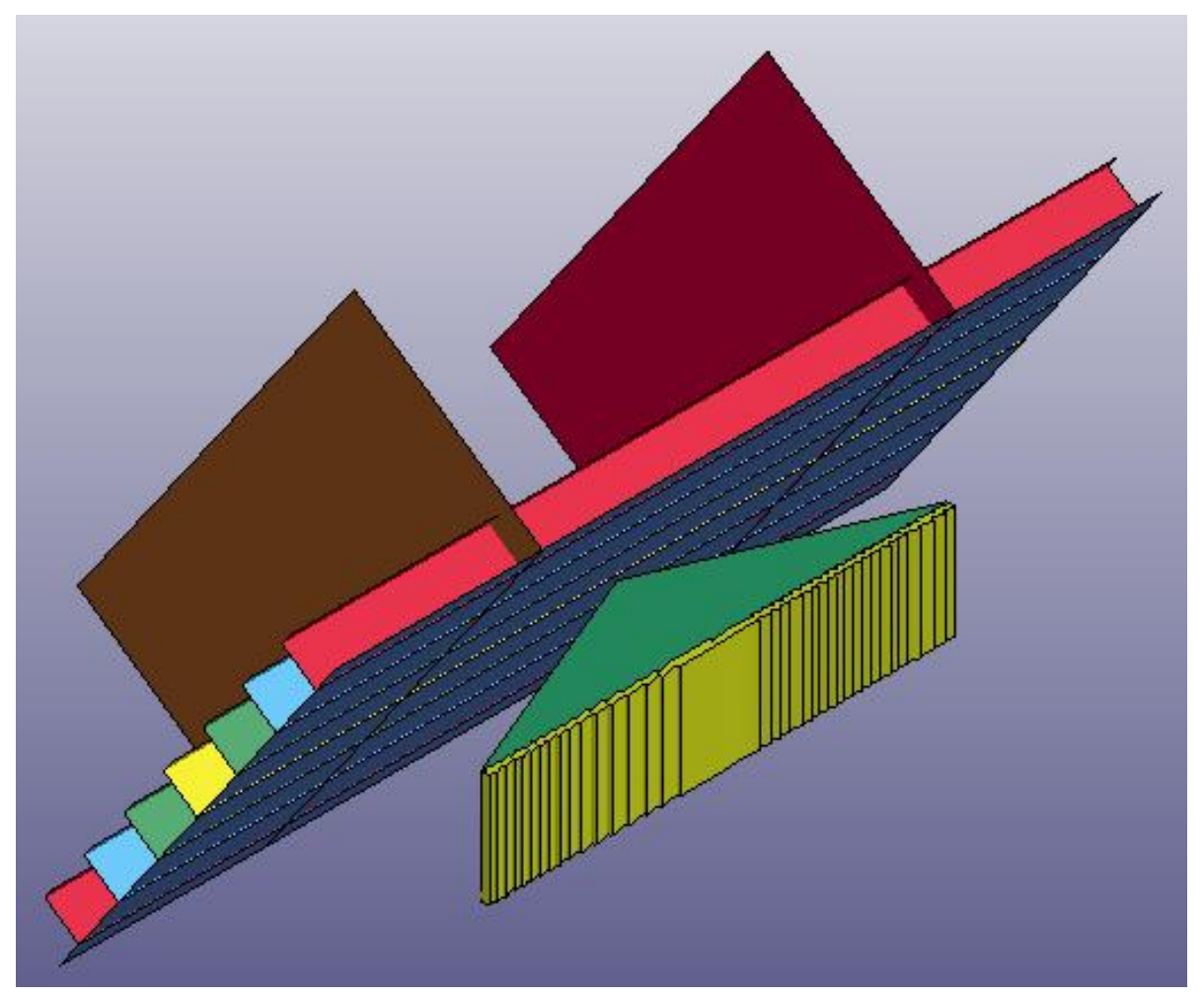

Figure 4.20 Grillage-Ice Interaction FEA Model 
The patch load benchmarking analyses examined two different loading scenarios, the ice interaction also similarly investigated an impact on and between frames, in addition to two different initial velocities to compare the algorithm's modelling capabilities of an elastic and plastic/collapse structural response. To produce an elastic structural response, the algorithm is set with an initial ship velocity of $1.0 \mathrm{~m} / \mathrm{s}$ and the ice with an $-0.1 \mathrm{~m} / \mathrm{s}$ velocity, producing a relative velocity of $1.1 \mathrm{~m} / \mathrm{s}$. With the relative velocity of $1.1 \mathrm{~m} / \mathrm{s}$, the algorithm finds an initial kinetic energy of $4202 \mathrm{~J}$. The plastic structural response scenario assumes an initial ship velocity of $2.4 \mathrm{~m} / \mathrm{s}$, with the ice velocity remaining at $-0.1 \mathrm{~m} / \mathrm{s}$, producing a relative velocity of $2.5 \mathrm{~m} / \mathrm{s}$ and an initial kinetic energy of $18,930 \mathrm{~J}$.

To model the interaction scenarios with FEA, the grillage structure is fixed on all four edges, with the rigid ice (rear ice part) only free to translate in the y-direction towards the grillage. The added mass of the rigid ice and the initial velocity of the ice parts are adjusted to produce a similar initial kinetic energy as the algorithm. The FEA interaction is set to run for 0.075 seconds, allowing the ice piece enough time to reach the structure and rebound. For the elastic structural response, the initial ice velocity is set to $2.8 \mathrm{~m} / \mathrm{s}$ with a total ice mass of $1085 \mathrm{~kg}$. The plastic interaction assumes an initial ice velocity of $5.9 \mathrm{~m} / \mathrm{s}$ and a total ice mass of $1085 \mathrm{~kg}$, with $875 \mathrm{~kg}$ of added mass added to the rigid ice part to achieve the correct initial kinetic energy.

To create the ice piece, two different material models are used. The green (front) portion of the ice is modelled using Gagnon's [62] crushable foam model and is not fixed in any degree. The yellow (rear) ice part is modelled as a rigid body with the same material properties as the crushable foam model and constrained in all directions except translation 
in the y-direction. The ice material models assume a density of $900 \mathrm{~kg} / \mathrm{m}^{3}$, a Young's Modulus of $9000 \mathrm{MPa}$, a Poisson's ratio of 0.003 , and a tensile cut-off of $800 \mathrm{MPa}$. The crushable foam material model follows the stress-strain curve shown in Figure 4.21. Crushable foam is used as the material model since ice deformation is not recoverable once the load is removed, similar to foam. Once a stress of $0.1 \mathrm{MPa}$ is reached on the stressstrain curve (Figure 4.21), the stress does not increase further until a strain of 0.065 is attained. Once the volumetric strain of 0.065 is achieved, the stress starts rising again following a steep fixed rate until a stress of $50 \mathrm{MPa}$ is met and remains at that stress for the rest of the interaction until the tensile cut-off is reached.
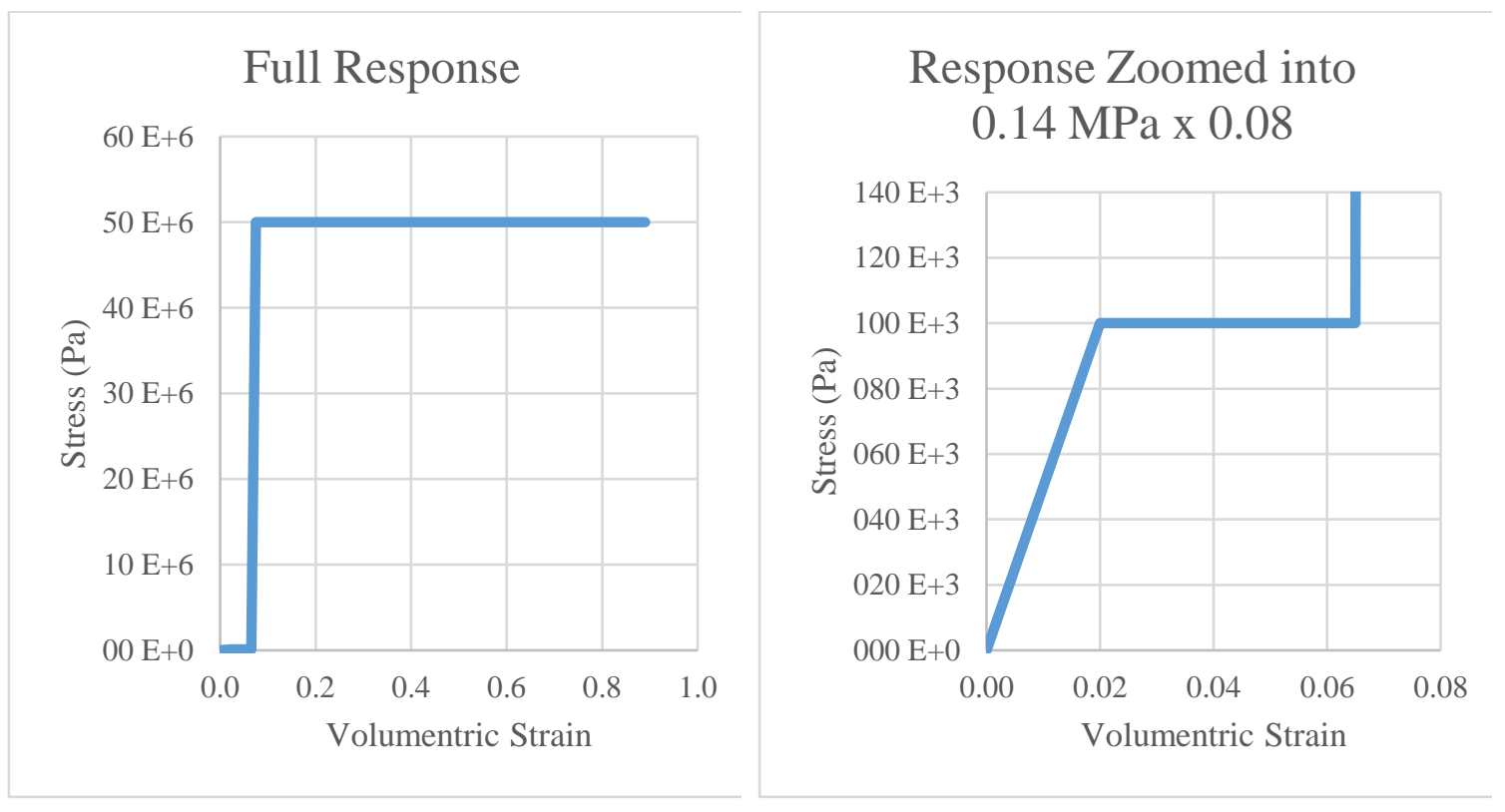

Figure 4.21 Crushable Foam Stress-Strain Curve

Finally, with the FEA models created the mesh convergence analyses are conducted. New mesh convergence analyses are conducted for each material response range and loading scenario. For both the elastic and plastic material responses for an impact on the frame the grillage mesh elements edges are set to $10 \mathrm{~mm}$ around the impact region, and for the elastic 
response, the ice piece mesh elements are set to $10 \mathrm{~mm}$ edge length. For the plastic response, if the elements are too small, this reduces the capacity of the crushable foam material model, therefore the ice elements had to be modelled with $12.5 \mathrm{~mm}$ edge lengths. From the ice interaction between frames, a mesh element edge length of $12.5 \mathrm{~mm}$ around the point of contact and the ice piece are used to model both material response ranges.

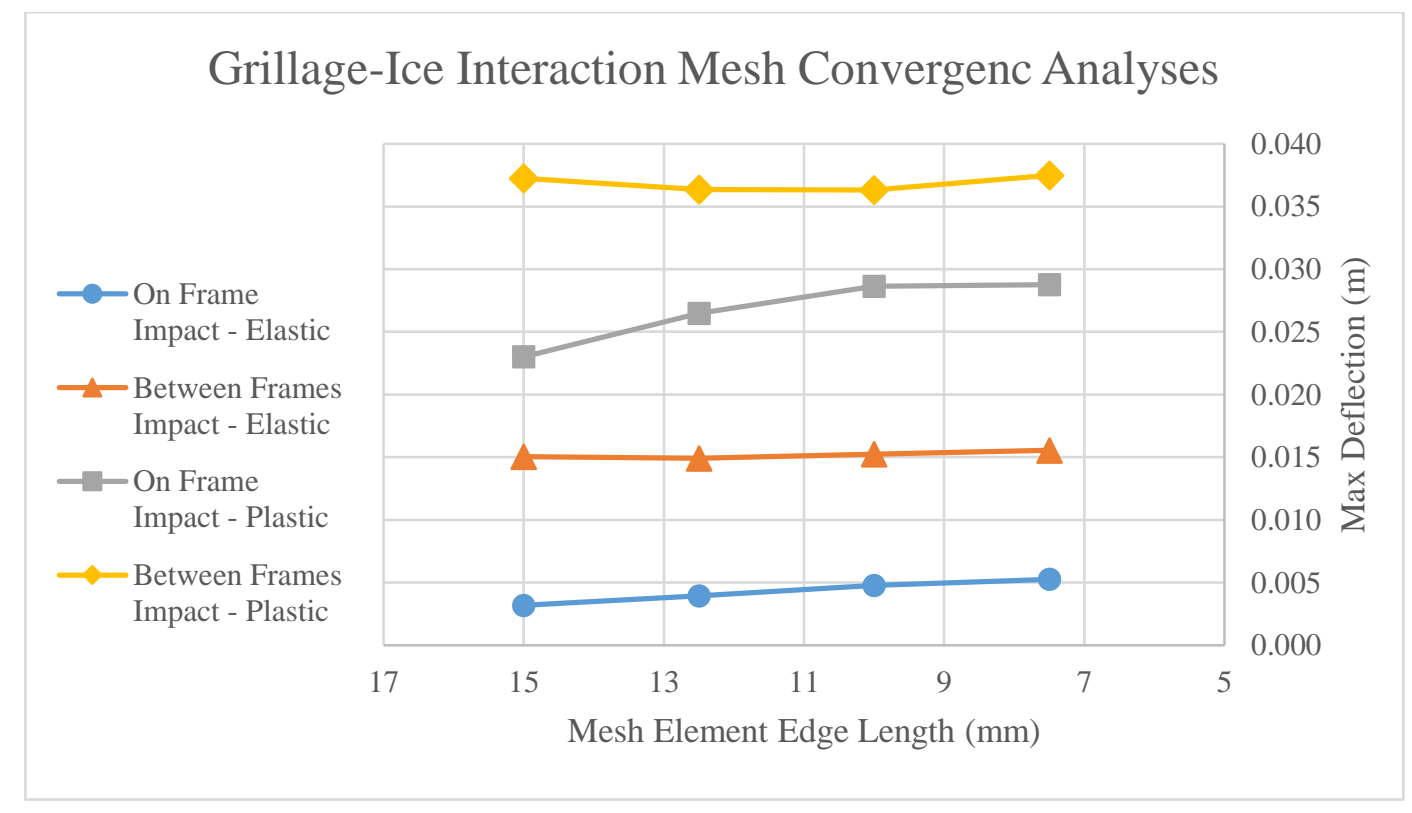

Figure 4.22 Grillage-Ice Interaction Mesh Convergence Analyses

\subsubsection{Ice Interaction Analysis}

First, the structural response of the elastic and plastic interactions is examined to determine if there is a difference in the prediction capabilities depending on the material response range. Both algorithm responses seem to under-predict the interaction, in the sense of the maximum contact force and amount of energy absorbed by the structure during the interaction. The elastic interaction, shown in Figure 4.23, models the interaction up to the point of the peak contact force between the structure and the ice. For the elastic interaction, 
the algorithm closely follows the FEA ice impact on the frame, but both the energy absorbed by the structure and the contact force terminates much sooner than the FEA model.

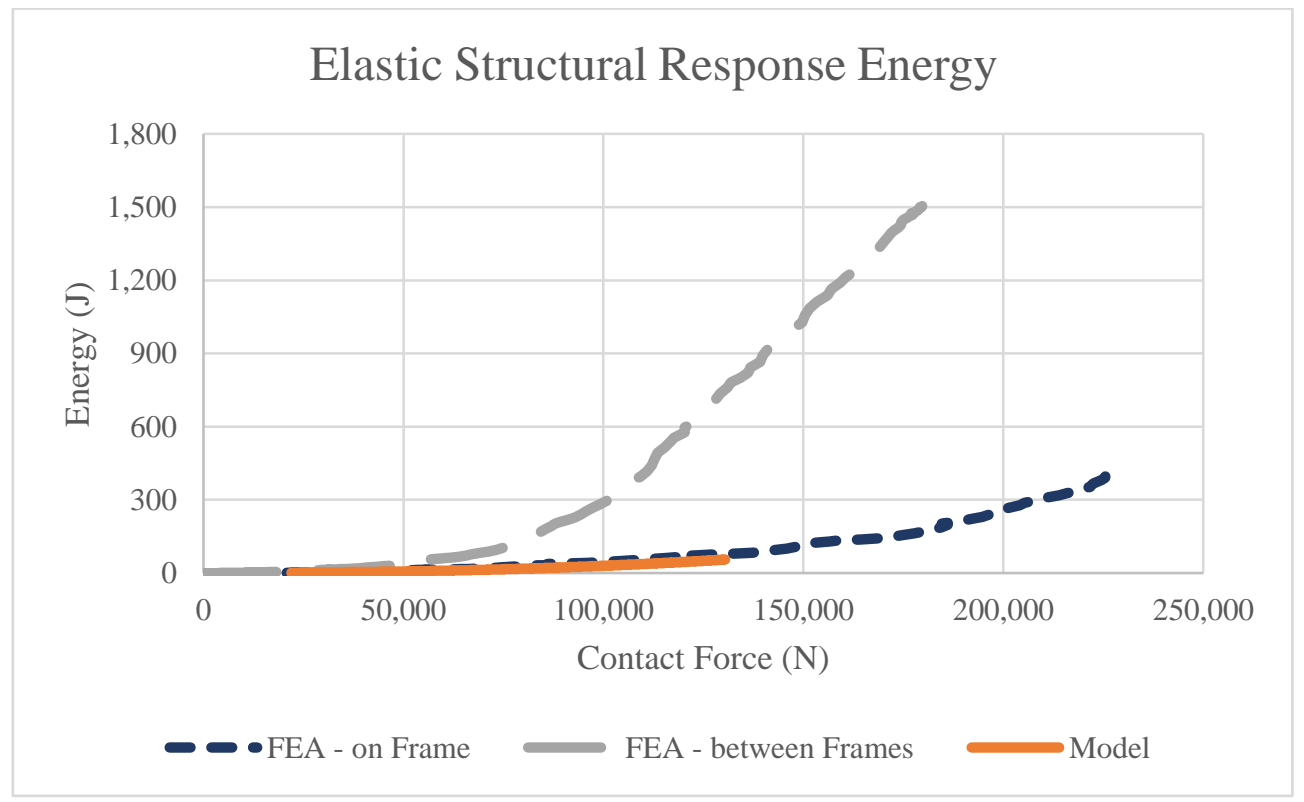

Figure 4.23 Elastic Grillage-Ice Interaction Structural Response

The plastic interaction assumes slightly more kinetic energy than the algorithm estimates the ice and structure can absorb before the termination limit state of 3-hinge collapse is reached. Both the algorithm and FEA model are set with an initial kinetic energy of 18,938 $\mathrm{J}$, but the algorithm finds that the structure and ice absorb up to $18,249 \mathrm{~J}$ before the algorithm terminates. Even when accounting for the FEA plastic structural response modelling the full interaction, Figure 4.24 still follows the same trends as the elastic analysis. The plastic model follows more closely the ice impact on the frame, with a slight under-prediction of the energy response. Again, the algorithm terminates much sooner and absorbs a lot less structural energy than assumed by the FEA models. 


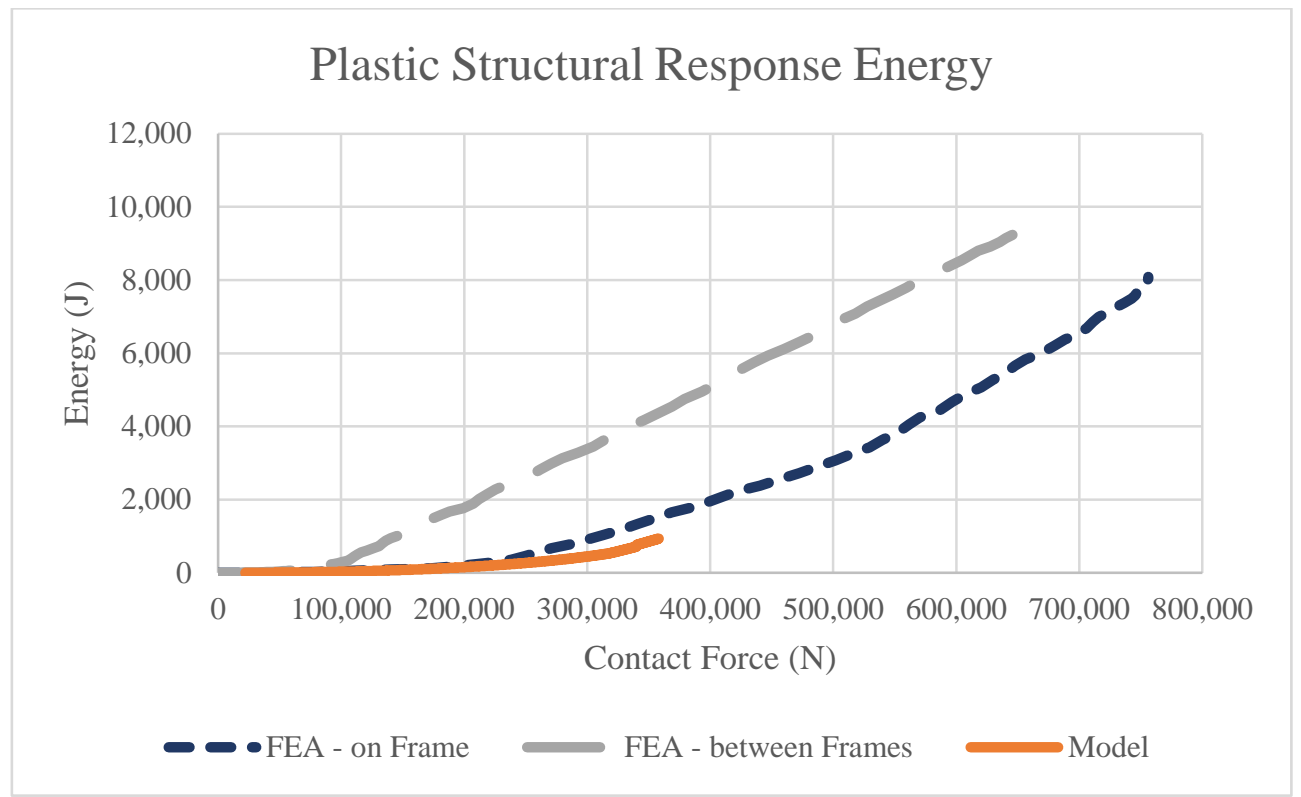

Figure 4.24 Plastic Grillage-Ice Interaction Structural Response

Since there is not a significant difference in the response between the elastic and plastic structural responses the rest of the benchmarking analyses only examines the plastic interaction since that response includes both the elastic and plastic material responses. When just the beam response is examined, in Figure 4.25, the algorithm more closely follows the FEA response of an ice impact between frames, modelled as the energy of the two frames on either side of the impact. For an impact directly on the frame, the beam model appears to be under-predicting the energy that the single-center frame produces. Again, the structural response has a much lower maximum contact force compared to the FEA responses, thus lowering the possible maximum energy absorption of the frame. 


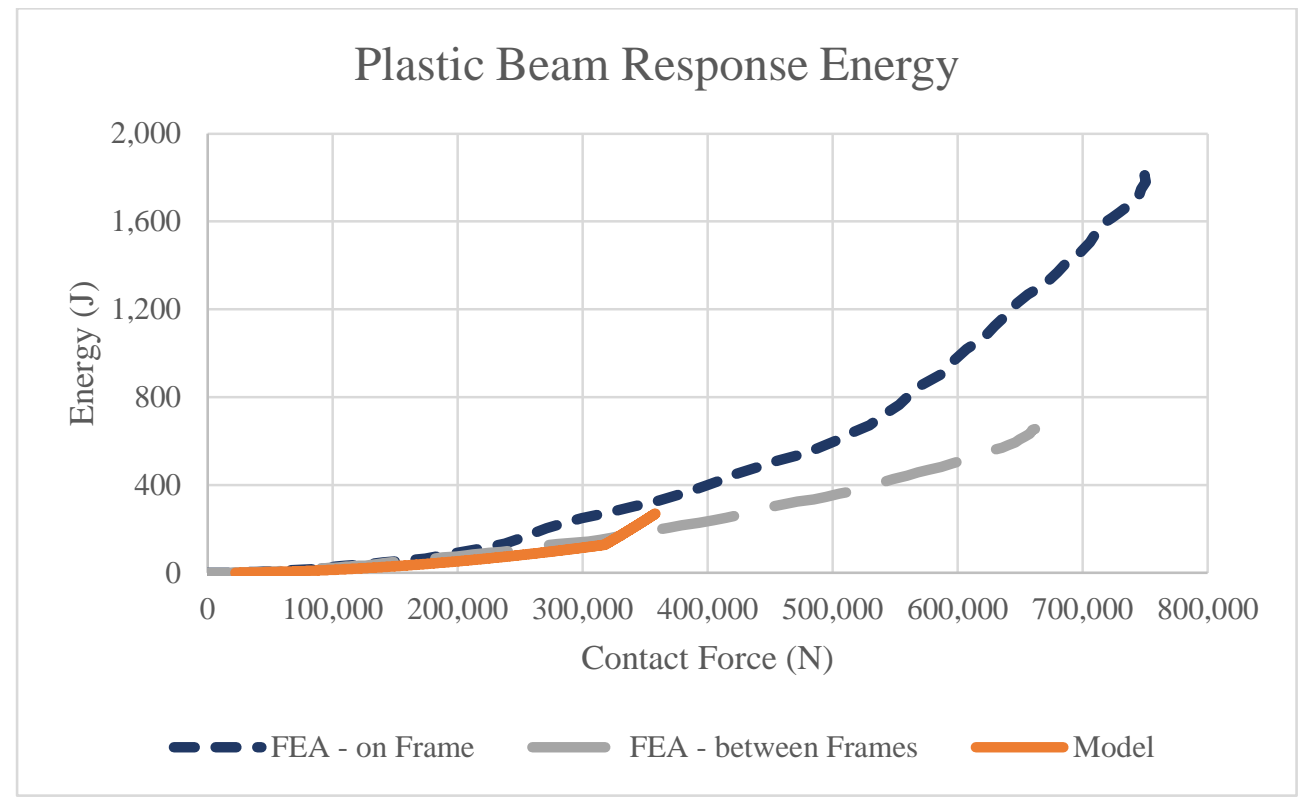

Figure 4.25 Grillage-Ice Interaction Plastic Beam Response

Next, the plate response is examined, Figure 4.26. The algorithm seems to be following the FEA model for ice impact directly on the frame the closest, again with the algorithm slightly under-predicting the energy response. Similar to the patch load analysis, the ice impact between frames appears to be experiencing a much greater energy response than the plate meta-model is predicting, likely due to the greater capacity of the large plate compared to the meta-model's design scenario. Unlike the patch load analysis for an impact on the frame, the algorithm does not over-predict the response but rather slightly under-predicts the response. These different response trends are likely due to the effect of the assumed fixed large patch load of the patch load analyses, compared to the relatively smaller varying rectangular patch load heights calculated from the wedge normal to the hull ice piece contact geometry and contact force. 


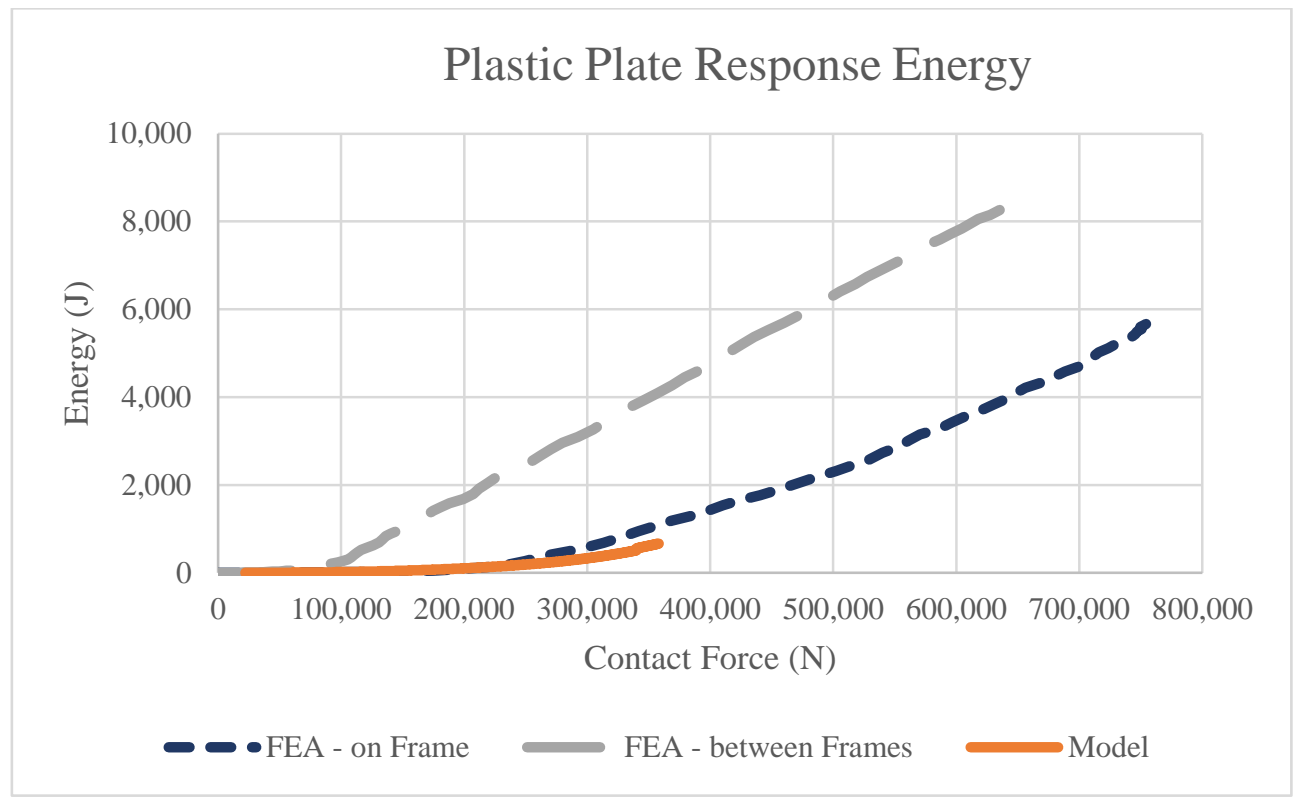

Figure 4.26 Grillage-Ice Interaction Plastic Plate Response

Lastly, the ice response is examined, Figure 4.27. Both the impact on and between the frames have a similar response, but the pressure-area relationship employed in the algorithm assumes the ice is absorbing a lot more energy than the crushable foam material model used in the FEA analyses. Since both the FEA and the algorithm are employing simplified ice-crushing models, both are only estimations of a true ice-crushing response and neither can really be considered more correct than the other. With that said, the more energy the ice absorbs the higher the contact force the structure can withstand, therefore the ice model assumed in the algorithm allows for higher impact energies. Nonetheless, if stronger ice is desired that more closely resembles the crushable foam model used in the FEA, the nominal ice strength and ice exponent can be adjusted. For this comparison, the algorithm assumes a nominal ice strength of $3 \mathrm{MPa}$ and an ice exponent of 0.2 . 


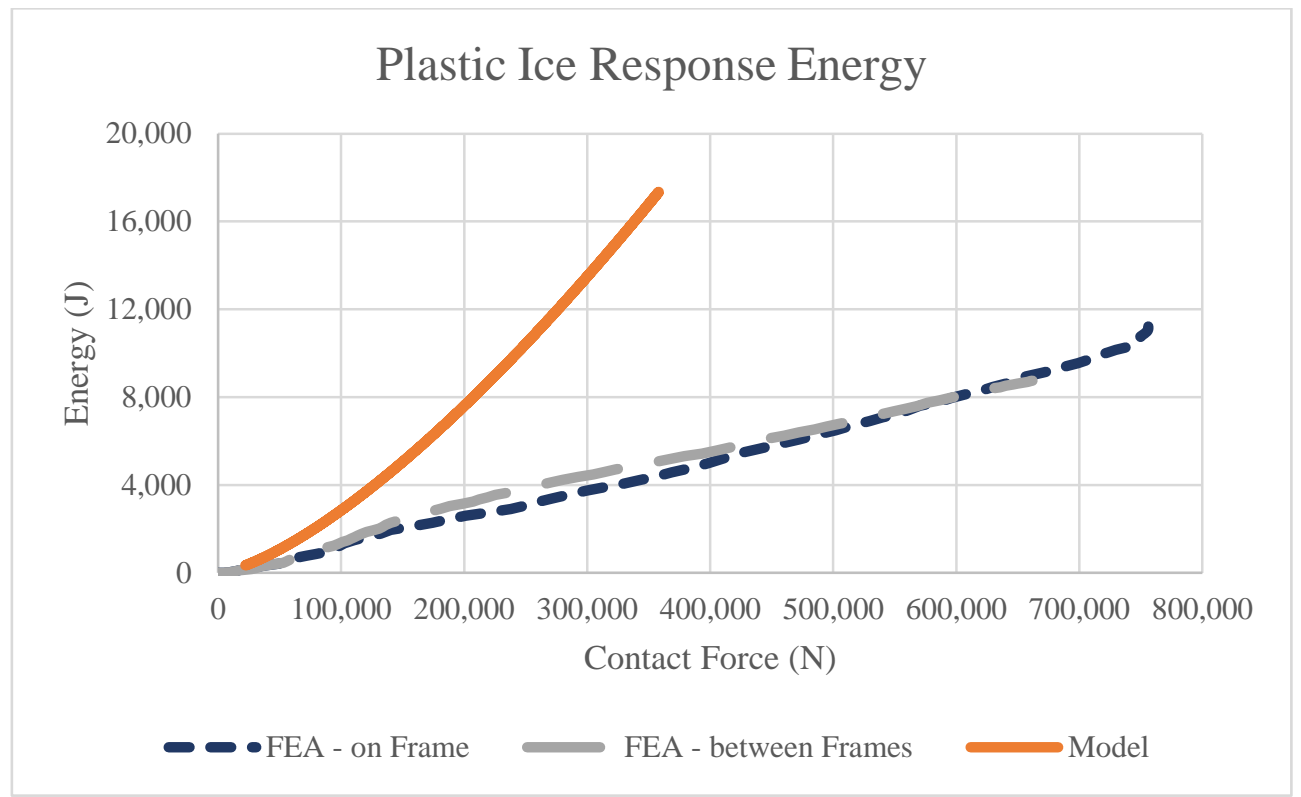

Figure 4.27 Grillage-Ice Interaction Plastic Ice Response

Now the onset of yield, indicating the transition from the elastic models to the plastic models, in the frame and plate models are investigated. Similar to the patch load analysis, due to the irregularity of the onset of yield in the grillage structure, the loads are only estimations based on notable concentrations of yield in the structural element. Examining Table 4.11, both the plate and the frame model estimated loads to cause yield, during the plastic interaction, fall in between the two different FEA loading events. For the ice impact on the frame, the algorithm over-predicts the load to cause yield in both the plate and beam, compared to an impact between the frames, where the model under-predicts the load.

Table 4.11 Grillage-Ice - Load at Yield (Plastic Interaction)

\begin{tabular}{|l|r|r|}
\hline \multirow{2}{*}{} & \multicolumn{2}{|l|}{ Initial Yield Load (N) } \\
\cline { 2 - 3 } & Plate & \multicolumn{1}{c|}{ Beam } \\
\hline Model & 340,310 & 317,510 \\
\hline FEA - on & 120,277 & 259,799 \\
\hline FEA - between & 492,861 & 548,438 \\
\hline
\end{tabular}


In addition, the peak energy at collapse is examined. With the FEA models, the maximum energy is recorded at the time-step of the minimum kinetic energy of the interaction, which is compared to the algorithm energy at the termination limit state of 3-hinge collapse. The collapse energy for both the elastic and plastic interactions is examined, to check that the error of the plastic interaction is not exaggerated due to the premature termination of the algorithm compared to the plastic FEA models. Examining Table 4.12, the early termination of the plastic model due to the plate collapse does not appear to be the cause of the high collapse energy error. Both the elastic and plastic interactions have a very similar percent difference.

Table 4.12 Grillage-Ice - Structural Termination Energy

\begin{tabular}{|l|r|r|r|r|}
\hline & Elastic Interaction & $\begin{array}{l}\text { Percent } \\
\text { Difference }\end{array}$ & Plastic Interaction & $\begin{array}{l}\text { Percent } \\
\text { Difference }\end{array}$ \\
\hline Model & 55.4 & & 935.0 & \\
\hline FEA - on & 410.9 & $87 \%$ & 8,138 & $89 \%$ \\
\hline FEA - between & 1,513 & $96 \%$ & 9,647 & $91 \%$ \\
\hline
\end{tabular}

Table 4.13 examines the significant difference between the algorithm's and FEA models' collapse energies, which are likely due to the significant difference in the absorptions of the ice models. Table 4.13 compares the initial kinetic energy put into the system, the collapse energies of the structure, the ice and total internal energy of the system. When the total internal energies at collapse are examined, it can be observed that all three models do have similar peak total internal energy, but the FEA models and algorithm distribute the energy differently, due to the different ice models. This difference in the structural collapse energy has the potential to be improved, with the adjustment of the ice model. 
Table 4.13 Grillage-Ice - Plastic Collapse Energy Responses

\begin{tabular}{|c|c|c|c|c|}
\hline & $\begin{array}{l}\text { Initial Kinetic } \\
\text { Energy }\end{array}$ & $\begin{array}{l}\text { Structural } \\
\text { Energy }\end{array}$ & $\begin{array}{l}\text { Ice-Crushing } \\
\text { Energy }\end{array}$ & $\begin{array}{l}\text { Total Internal } \\
\text { Energy at Collapse }\end{array}$ \\
\hline Model & 18,938 & 935.0 & 17,349 & 18,284 \\
\hline FEA - on & 18,936 & $8,137.6$ & 11,592 & 19,730 \\
\hline FEA - between & 18,936 & $9,647.2$ & $8,762.8$ & 18,410 \\
\hline
\end{tabular}

Table 4.14 puts the trends observed in the previous figures into numbers, by comparing the energy estimations dependent on the contact force of the different components and total structural response up to the algorithm's maximum contact force. The beam response overall is okay. The beam model produces a higher mean error when compared to the ice load on the frame, but the error is more consistent, in comparison to the ice impact between frames, which has a lower error but has a greater variance and standard deviation. The plate meta-model has a similar trend to the beam model, in the sense that the smaller average error has a higher variance and standard error, but in this case, the ice load on the frame produces the lower mean percent difference. The higher error associated with ice impact between the frames is likely due to the increased plate response that is not accounted for by the plate meta-model, as similarly observed during the grillage patch load analyses. The comparison of the ice models for both loading conditions is very poor, in all regards.

Finally, the total structural response of the full grillage is compared to the combined energy response of the algorithm. For the ice impact on the frame, the response has an okay percent difference of about $34 \%$, with higher variance and standard deviation. The ice load between the frames has a very high error, likely due to the very high plate energy error, but with much lower variance and standard deviation, allowing for a potential correction term. 
Table 4.14 Grillage-Ice Energy Prediction Statistical Analysis

\begin{tabular}{|c|l|r|r|r|}
\hline & $\begin{array}{l}\text { Impact location } \\
\text { Relative to Frame }\end{array}$ & \multicolumn{1}{l|}{$\begin{array}{l}\text { Mean } \\
\text { Error }\end{array}$} & $\begin{array}{l}\text { Variance } \\
\text { Error }\end{array}$ & \multicolumn{2}{l}{$\begin{array}{l}\text { Standard Deviation } \\
\text { Error }\end{array}$} \\
\hline \multirow{3}{*}{ Beam } & On & $42.6 \%$ & $67.5 \%$ & $8.22 \%$ \\
\cline { 2 - 5 } & Between & $33.8 \%$ & $76.6 \%$ & $8.75 \%$ \\
\hline \multirow{3}{*}{ Plate } & On & $32.9 \%$ & $327 \%$ & $18.1 \%$ \\
\cline { 2 - 5 } Ice & Between & $91.8 \%$ & $10.8 \%$ & $3.29 \%$ \\
\cline { 2 - 5 } & On & $178 \%$ & $3382 \%$ & $58.2 \%$ \\
\hline $\begin{array}{c}\text { Total Structural } \\
\text { Response }\end{array}$ & Between & $141 \%$ & $1606 \%$ & $40.1 \%$ \\
\cline { 2 - 5 } & Between & $33.7 \%$ & $112 \%$ & $10.6 \%$ \\
\hline
\end{tabular}

Similar to the grillage patch load analyses, a box and whisker plot (Figure 4.28) is created to visualize and put into perspective the date shown in Table 4.14. Each box shows the percent difference of the energy estimation between the algorithm and the plastic FEA loading conditions depending on the contact force. The plot shows the errors associated with the beam and plate structural components, in addition to the total structural response, for both impact locations on the grillage. The ice response is removed due to its disproportionally large error, overshadowing the more important structural analyses.

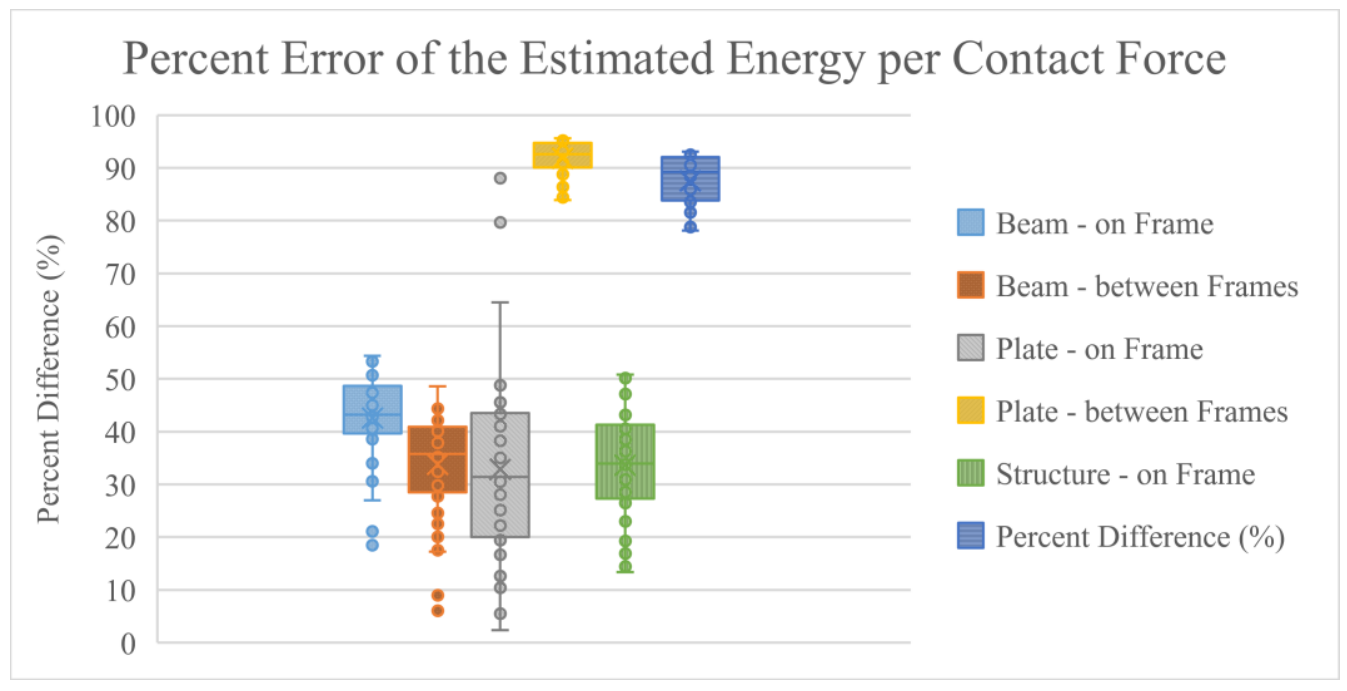

Figure 4.28 Grillage-Ice Energy Prediction Box and Whisker Plot 


\subsubsection{Ice-Grillage Interaction Summary}

Overall, when the algorithm's response is compared to the FEA models within the algorithm's predicted response range, the algorithm has an acceptable agreement, especially for an ice impact directly on the frame. The response for an ice impact between frames does not have as good of an average error, but most of the error can be attributed to the plate model, which could be improved with a more detailed plate model or with a correction term directly in the algorithm. Finally, the most significant difference between the algorithm and the FEA models is the collapse or peak structural response energy of the interaction. The difference in the collapse energies can be associated with the increased energy absorption of the pressure-area relationship ice-crushing model. 


\section{Chapter 5 Discussion}

There are some models with similar methodologies to the one presented here, for example, Amdahl [37] knowing the force acting on the ice and the structure must be equal, equates the ice force and the structural resistance. But typically, these shared-energy approaches model the external mechanics analytically and model the internal mechanics with nonlinear FEA [63], to allow for the modelling of the interaction effects like the wrapping of the structure around the ice and changes in the ice edge geometry [37]. This algorithm attempts to model the internal mechanics analytically and includes some interaction effects between the structure and ice, by considering the changing contact geometry during an interaction by modelling variable patch load dimensions. The patch load dimensions vary depending on the contact force and hull angles at the location of impact. This approach is also more realistic than the design patch load of the IACS Polar rules [1], which assumes a fixed patch load based on the hull angles and displacement of the ship, which removes any dependence of the patch load on the ice piece.

To create this algorithm several assumptions are implemented to simplify the complex nature of a coupled shared-energy approach. The main assumptions investigated during the benchmarking process are the methods used to model the structural response and the modelling capabilities of the algorithm as a whole. To model the structural response of a grillage there are few different approaches. The most common analytical approach, and the method employed with this algorithm, is to model the structural elements individually and then superimpose the linear responses, but with this approach, the interaction effects between the structural components are not accounted for, thus the components are assumed 
to independently contribute to the total structural resistance $[25,33,39]$. The IACS Polar rules, which the majority of the assumptions and methods for this algorithm stem from, combined two approaches. Both plate and frame models are examined independently, but to also consider some interaction effects the plate is modelled as a flange in the frame analysis. With this approach, the plate is modelled as a large flange attached to the bottom of the frame, where the plate flange spans the distance between the frames $[35,45]$. Therefore, this discussion starts by examining the individual structural models, followed by an evaluation of the algorithm as a whole compared to the grillage FEA benchmarking.

\subsection{Isolated Structural Models}

The benchmarking analysis did present some errors with the simplified beam model, particularly with the plastic response model. One cause of the error associated with the plastic model can be attributed to the early transition between the elastic and plastic models that produced about a $12 \%$ difference between the transition loads of the algorithm and FEA. Part of the difference between the transition loads might stem from the beam model ignoring the partial formation of the plastic hinges, which ignores a transition phase and jumps directly from the elastic to the plastic response. The beam model assumes a discrete transition between the elastic to the plastic response once the ends of the elastic beam reach full through-thickness yield, at which point the beam model switches to the plastic response with the formation of the plastic hinges modelling the yielded ends of the beam. Therefore, this discrete transition between beam response models does not compare to the more gradual formation of the plastic hinges of the FEA models. Similarly, the early termination 
of the beam model is likely also affected by the neglect of the partial hinges at the center of the beam.

Some of the differences noted during the comparisons in the models might also stem from errors associated with the FEA models. For example, with the elastic perfectly plastic material model assumed with the beam FE analyses, as the element size decreases the error associated with the plastic edge hinges increases. As the size of beam elements decrease the plastic edge hinge occur sooner, since the average stress of the end element increases as the element size decreases. Another issue with the FEA model analyzed during the beam benchmarking process is the element edge at the center of the beam, opposed to a singular element centred on the beam. One element centred on the beam models the average onset of yield better, which could affect the termination load and energy comparison of the model.

The error associated with the maximum energy of the beam at the termination limit state of 3-hinge collapse is also likely affected by the early transition and termination of the model when compared to the FE analyses, in addition to some of the minor energy mechanisms being ignored. The collapse energy of the beam model was examined against the FEA internal energy and the 3-hinge collapse energy of the traditional beam energy methods. Technically, the FEA model is more accurate than the conservative estimation of the 3hinge collapse plastic hinge model. Compared to the FEA and simplified analytical 3-hinge collapse models, the beam model created for this algorithm under-predicted the energy at termination but is much closer to the accepted simplified analytical model. Both the analytical 3-hinge collapse model and the full beam response model terminate at the same contact force, therefore the error associated with the termination load would not affect the 
error between the models. The difference between the models is likely because the algorithm only considers the bending strain energy and rotational work of the plastic edge hinges, leaving the energy mechanisms like axial strain, energy from shear stress, and energy from the formation of the partial edge hinges unaccounted for.

Within the bounds of the grillage structure, the beam model performs very similarly to its individual evaluation, finding that the cross-section of the frame does not affect the performance of the model. For the fixed patch load (Figure 4.16) the switch between the elastic and plastic model can be observed to help the energy response adjust and approach the FEA response. The center frames of the FEA grillage-ice interaction also have a similar response to the beam model (Figure 4.25). The beam model slightly under-predicts the energy response of ice impact on the frame, likely due to the smaller patch load lengths, which were found to have poorer prediction capabilities during the benchmarking analyses.

The plate model, in contrast, had much better agreement with the individual FEA models during the benchmarking analyses. The largest errors associated with the plate meta-models were from the low contact forces of the elastic response model, which is not a huge concern since by the time the more critical contact forces and energies are being modelled the difference in the responses has greatly decreased. The plastic meta-model had great agreement with the FE analysis, which is likely due to the smaller response surface compared to the elastic model. The purpose of splitting the plate response into two models was to decrease the size of the meta-models response surface, which did not necessarily produce an even split, but it did help improve the more critical of the two models. Due to the nature of regression models, like the plate meta-models, the transition and termination 
loads are under-predicted with lower patch load heights and over-predicted with large patch load heights; the collapse energy of the plate follows the same trends as the termination loads.

When just the plate response was examined within the boundaries of the grillage structure, the very specific design scenario of the plate model produces some interesting observations. With the fixed patch load analysis (Figure 4.17), it was found that the smaller plate, assumed with the creation of the meta-model, greatly under-predicts the full membrane capacity of the plate for the patch load centred between the frames. In contrast, for the patch load centred on the frame, the meta-model over-predicts the plate response since the model does not consider the reduced plate response due to interaction effects of the frame. Therefore, with the fixed patch load the plate meta-model response falls in between the two possible loading events on a grillage. With the grillage-ice interaction (Figure 4.26) the algorithm produces smaller patch load heights than the fixed patch load model assumed, which finds the plate model more closely resembled the loading of an ice piece on the frame. Even with the grillage-ice interaction for an impact between frames, the plate model still greatly under-predicted the capacity of the larger grillage plate. These trends suggest that the design scenario for the meta-model is acceptable for an impact on the frame but does not allow for the full membrane effect of a larger plate to properly reflect the interaction. Additionally, it should be noted that the large plate within the grillage has an aspect ratio more closely resembling a long plate, compared to the meta-model design scenario of a plate with a 2:1 aspect ratio. This difference in the aspect ratio affects the 
collapse mechanisms produced during the interaction, which is likely also contributing to the large error associated with the loading event between the grillage frames.

\subsection{Assembled Algorithm}

The FEA grillage structure benchmarking analyses are conducted for a number of reasons. Similar to Daley and Hermanski's [53] large grillage experiments, the FEA grillage analysis allows the algorithm to be examined against more realistic boundary conditions, while still focusing on the center frame(s) and plate response of the interaction. The FEA grillage benchmarking analysis also allows the assessment of the weakly coupled analytical approach of the algorithm, against the fully coupled approach of the FEA grillage-ice interaction [29].

From the fixed patch load analysis, it was found that depending on the loading event, only either the frame or plate experiences yield, contrary to the algorithm, which assumes that both structural elements experience the same loading conditions. In reality, depending on the loading event, the energy is distributed differently between the structural elements within the grillage structure. The algorithm does not account for the variation in the structural response. For example, similar to Daley and Hermanski [53, 64], the fixed patch load grillage benchmarking analysis found that the frame, within the grillage structure, has an increased capacity compared to the isolated frame model adopted in the algorithm. Within the grillage structure, both the frame and plate withstood much higher loads, indicating the support of neighbouring frames, due to an energy distribution across the grillage structure that the algorithm does not consider. Also, generally, grillage structures 
and structural elements do not yield as uniformly as described and assumed with analytical isolated models, which results in variations in the yield loads.

The plate model seems to be more affected by being modelled as an isolated structural element compared to the beam model, as the plate model over and under-predicts the energy response depending on the location of the impact on the grillage. The plate model could be improved with the addition of an effective thickness term, for an impact on the frame, or an enhanced membrane response term for an impact between frames. Modelling a plate with an effective thickness term accounts for the inclusion of a frame by proportionally increasing the thickness of the plate [25].

The final benchmarking analysis assesses the algorithm's ability to model variable contact from an ice piece interaction. The main findings from the analysis are that the ice used in the modelling of the FEA and the algorithm absorbed very different amounts of energy. According to $\operatorname{Kim}[9,17]$, they found that the majority, about $70-80 \%$, of the energy during an ice-structure interaction is dissipated through ice-crushing. The pressure-area relationship of the algorithm estimated that $95 \%$ of the energy is used in ice-crushing, compared to the FEA models that found the ice only absorbed about $50 \%$ of the collision energy. Both the algorithm and the FEA have a similarly large error relative to the energy ratio, but the overestimation of the energy absorbed by the ice-crushing of the algorithm could result in the overestimation of the ship structure resistance, compared to the FEA ice model that produces a more conservative estimation of the structural response.

There are conflicting opinions regarding the application of Gagnon's crushable foam ice model [62], which is applied in the grillage-ice interaction FEA models. Liu et al. [65] 
found that the crushable foam model lacks physical explanation and does not model cracks and damage produced during a typical ice-crushing interaction. In contrast, Han et al. [66] found that in general the crushable foam, with a volumetric hardening function, is the most suitable ice material and typically used as an energy absorption structure. Nonetheless, even with the discrepancies between the ice material models, the general algorithm response up to its early termination has a similar response error when compared to the other benchmarking analyses for an impact on the frame, suggesting the algorithm as a whole has the potential to model more realistic loading scenarios.

The ice-structure response is very dependent on the contact geometry produced by the local ice shape and structural arrangement, as well as the mechanical properties and velocity of the ice [17]. The change in the contact geometry will vary the energy used during the icecrushing process and the structural resistance of the ship during any given ship-ice interaction. With that said, if the ice-structure interaction is so strongly influenced by the contact geometry, the interaction is also strongly affected by the coupling approach of the system. Yu et al. [29] noted that a weakly coupled approach is more suited with blunt ice interactions, since there is less change in contact geometry from ice-crushing and spalling events. A wedge ice piece is assumed with both the algorithm and benchmarking models. Some of the errors associated with the ice responses might also be affected by the modelling approach for the changing contact geometry. However, the weakly coupled system of the algorithm absorbs more energy than the FEA models, which is typically associated with an increased rate of ice-crushing and change in contact geometry. 
As a whole, the algorithm has reasonable modelling capabilities. The frame model is missing some energy absorption mechanisms, but that is compensated by the slight overprediction of the plate response for an impact on the frame, in which the grillage response for an impact on the frame has the lowest average prediction error of around $25 \%$. The algorithm greatly under-predicts the structural response for an impact between frames, which is mainly attributed to the high under-prediction of the plate response, since the frame response is relatively similar for both loading events. The errors associated with the plate model have the potential to be corrected with a more comprehensive meta-model. 


\section{Chapter 6 Conclusion}

An analytical shared-energy approach is presented and investigated to assess the algorithm's ability to model a ship-ice interaction for low- and non-ice-class ships. Previously, to model a ship-ice interaction one of two approaches are adopted. For a higher ice-class ship or icebreaker, a strength design approach is acceptable, where the structure is designed to withstand the crushing strength of the ice. This approach was also adopted by the IACS polar rules, which determines the appropriate ice contact force depending on the structural arrangement and desired ice-class of the ship. Alternatively, the other common method to model a ship-ice interaction is with a shared-energy approach. When the structural response is included in the energy response, the most common approach to model the interaction is with nonlinear FEA, since it allows for the modelling of the changing contact geometry and effects like wrapping.

A modular algorithm design is employed to create a hybrid-analytical model, where each different module or component of the algorithm modelled a different component of the system. This modular approach allows for simple interchanging of models, as the user sees fit, and replacement of new models as analytical modelling capabilities improve. The material response range of the algorithm is designed to model the structural response up to the IACS Polar rules design load of 3-hinge collapse. In addition, some other methodologies drawn from the IACS Polar rules to create the algorithm include modelling the structural elements of a grillage structure in isolation, assuming a Popov type collision model, and assuming the ice primarily fails due to ice-crushing. 
To determine the initial kinetic energy of the colliding system, a Popov collision model is employed. To model the ice-crushing energy, Daley's ice-crushing force energy models are adopted. The structural response is represented by the superposition of the individual structural elements. To model the beam bending energy, classical beam theory is applied to derive the strain energy. The plate model is created with FEA experiments and the design of experiments methodology. The algorithm iterates through the shared contact force, between the ice and the structure, until the internal energy (the ice-crushing and structural response) equals the external energy (the initial kinetic energy).

The various benchmarking analyses were conducted to assess the modelling capabilities of the individual structural models, the superposition of the structural models against a grillage structure, and the algorithm as a whole. The beam model had great agreement in the elastic response range but under-predicted the plastic response and the collapse energy of the beam compared to the FEA models. The isolated plate meta-model had great agreement when compared to the FE benchmarking analysis. The largest error associated with the plate model was the lower elastic contact forces, which pose no real risk to the structure. When the structural response of the algorithm was assessed with a fixed patch load on a grillage structure, it is found that for an impact centred on a frame, the structural models had reasonable agreement, but for an impact centred between frames, the plate model does not properly capture the full capacity of the plate response.

Finally, FEA models were created to investigate the algorithm's ability to model an ice impact on the structure. The FEA grillage structure was impacted by a wedged ice piece with an initial velocity, constrained to only move in one direction. During the post-analysis 
it was found that the ice models used in the algorithm and the FEA models absorbed very different amounts of energy, affecting the maximum contact force even when both models were inputted with the same initial kinetic energy. Nonetheless, ignoring the early termination of the algorithm, the algorithm had a similar response to the FE model for the ice impact on the frame, and a comparable error to the patch load on the frame benchmarking analysis. The ice interaction also found for an ice impact centred between frames, that the plate meta-model greatly under-predicted the plate response. This underprediction of the plate response within the grillage structure is due to the smaller plate employed while conducting the load experiments, which reduces the effect of the membrane response compared to the larger plate in the grillage structure.

\subsection{Recommendations}

If more comprehensive interaction effects between the plate and frame are desired, the addition of the plate in the frame cross-section, as a flange, may allow for an improved response and account for some of the missing beam energy. However, while creating the beam model, only a quick assessment was conducted, with a single beam cross-section and length, to evaluate the assumption of ignoring the axial tension of the beam and considering only the bending response. Therefore, if cross-sections that are more complex or drastically different beam lengths, than those examined while creating the model, are to be modelled, a more extensive stress analysis of the beam is suggested.

There are two suggestions to improve the plate meta-model when compared to the grillage plate response. More factors could be added to the meta-model to create a more comprehensive plate model, like the patch load width, plate thickness, and loading location 
on/off the frame. Another option is to conduct DoE optimization experiments to determine the best plate model dimensions to model the plate response within a grillage structure. For example, some optimization criteria could be to better depict the membrane response of the plate, while not creating an unnecessarily large model. Conversely, the two options could be combined. A DoE optimization or factor screening experiment could be conducted to make sure only the important plate factors are included in the plate model, to avoid an excessively complex meta-model.

A revised ship-ice FEA analysis is also suggested with more comparable ice models. Overall, during the benchmarking assessment, ignoring the errors from the ice models, the algorithm did have similar modelling capabilities compared to the other benchmarking analyses. Nonetheless, a revised ship-ice FEA analysis is suggested, to make sure there are not any large errors being hidden by the even larger difference between the ice models. In regard to the ship-ice benchmarking analysis, it is also recommended that a proper patch load dimension analysis between the algorithm and the FEA be conducted. This assessment would be to determine if the modelling approach adopted in this algorithm is helping improve the weakly coupled approach used to model the interacting system. The patch load dimensions, relative to the contact force, of the algorithm and FEA grillage-ice interaction, should be compared. The rate of change of the patch load dimensions as the contact force increases could also be examined between the two models.

\subsection{Future Work}

Due to the nature of the modular algorithm, there are several ways to improve its modelling capabilities without any significant changes to the layout. Currently, the algorithm assumes 
a Popov collision to determine the kinetic energy introduced to the system. Over the years, more comprehensive external energy models have been created to model ship-ice interactions [36, 67], a more detailed model could replace the Popov collision model. In addition, more complicated interactions like sliding loads could be incorporated into the algorithm. Similarly, a more detailed ice-crushing response could be adopted to model nonuniform ice pressures, including properties like hard zones. Another approach to enhance the ice response is to include more ice failure mechanisms and criteria, like failure due to bending or cracking, which have much lower failure loads. The structural response of the algorithm could also be enhanced from a more comprehensive failure criterion, particularly the frame model, which currently assumes the frame only fails due to bending. The frame model could benefit from also checking for buckling, end frame loading, and shear hinge failure criteria, especially if the frame experiences more complex loads than the one assumes with this model. As mentioned previously, the plate meta-model could be improved by conducting optimization experiments to determine the best size plate to model a grillage plate response. To improve the weakly coupled modelling of the interaction effects of the ice and grillage, the algorithm could benefit from a more accurate changing contact geometry $[17,29]$. Also, effects like the wrapping of the structure around the ice could be taken into account, which affects the confinement of the ice and loading of the structure [37].

Finally, some suggestions are offered regarding implementing the algorithm into a simulator-type environment. To avoid the need for real-time computation of the structural response and techniques like numerical methods to improve the computational speed of the 
implicit system, a lookup table could be created with various types of preprocessed interactions. The extent of the interactions and details examined can vary and be arranged in a matrix type model, to rapidly notify the significance of the interaction to the user. The algorithm would be employed to compute the interactions with the specified structural arrangement, ice masses, and collision speeds, to determine what type of material response ranges the interaction falls in: elastic, plastic, or past the allowed design load. 


\section{Chapter 7 Bibliography}

[1] IACS, "Structural Requirements for Polar Class Ships," International Association of Classification Societies, 2019.

[2] Y. N. Popov, O. V. Faddeyev, D. Y. Kheysin and A. A. Yakovlev, "Strength of Ships Sailing in Ice (Technical Translation FSTC_HT_23_96_68)," Sudostroyeniye Publishing House, Leningrad, 1967.

[3] C. Daley, "Energy Based Ice Collision Forces," in 15th International Conference on Port and Ocean Engineering under Arctic Conditions, Helsinki, 1999.

[4] C. Daley and K. Riska, "Review of Ship-Ice Interaction Mechanics," FinnishCanadian Joint Research Project No. 5 "Ship Interaction With Actual Ice Conditions", Otaniemi, 1990.

[5] B. Su, K. Riska and T. Moan, "Numerical study of ice-induced loads on ship hulls," Marine Structures, vol. 24, no. 2, pp. 132-152, 2011.

[6] G. W. Timco and W. F. Weeks, "A review of the engineering properties of sea ice," Cold Regions Science and Technology, vol. 60, pp. 107-129, 2010.

[7] P. Kujala, "Ice Loading on Ship Hull," in Encyclopedia of Maritime and Offshore Engineering, J. Carlton, P. Jukes and Y. S. Choo, Eds., Hoboken, John Wiley \& Sons, Ltd., 2017. 
[8] J. Dolny, "Methodology for Defining Technical Safe Speeds for Light IceStrengthened Government Vessels Operating in Ice (SSC- 473)," Ship Structure Committee, Houston, 2018.

[9] E. Kim, M. Storheim, J. Amdahl, S. Løset and R. U. F. von Bock und Polach, "Laboratory experiments on shared-energy collisions between freshwater ice blocks and a floating steel structure," Ships and Offshore Structures, vol. 12, no. 4, pp. 530-544, 2017.

[10] A. Kendrick and C. Daley, "Structural Challenges Faced by Arctic Ships (SSC461)," Ship Structure Committee, Kanata, 2011.

[11] K. Riska and J. Kämäräinen, "A Review of Ice Loading and the Evolution of the Finnish-Swedish Ice Class Rules," Society of Naval Architecture and Marine Engineering, Houston, 2011.

[12] G. W. Timco and D. Sudom, "Revisiting the Sanderson pressure-area curve: Defining parameters that influence ice pressure," Cold Regions Science and Technology, vol. 95, pp. 53-66, 2013.

[13] I. J. Jordaan, "Mechanics of ice-structure interaction," Engineering Fracture Mechanics, vol. 68, no. 17-18, pp. 1923-1960, 2001. 
[14] E. Kim, W. Lu, R. Lubbard, S. Løset and J. Amdahl, "Toward a Holistic Load Model for Structures in Broken Ice," in 23rd International Conference on Port and Ocean Engineering under Arctic Conditions, Trondheim, 2015.

[15] K. Riska, "Ice edge failure process and modelling ice pressure," Philosophical Transactions A, vol. 376, no. 2129, 2018.

[16] C. G. Daley, "Reanalysis of Ice Pressure-Area Relationships," Marine Technology, vol. 44, no. 4, pp. 234-244, 2007.

[17] E. Kim, M. Storheim, J. Amdahl, S. Løset and R. von Bock und Polach, "Drop test of ice blocks on stiffened panels with different structural flexibility," in 6th International Conference on Collision and Grounding of Ships and Offshore Structures, Trondheim, 2013.

[18] E. M. Appolonov, A. V. Didkovsky, M. A. Kuteinikov and A. B. Nesterov, "Improvement in design models for ice load evaluation under vessel impact against ice," Ships and Offshore Structures, vol. 6, no. 3, pp. 249-256, 2011.

[19] E. Kim and J. Amdahl, "Discussion of assumptions behind rule-based ice loads due to crushing," Ocean Engineering, vol. 119, pp. 249-261, 2016.

[20] K. Riska and R. Bridges, "Limit state design and methodologies in ice class rules for ships and standards for Arctic offshore structures," Marine Structures, vol. 63, pp. 462-479, 2019. 
[21] K. R. Croasdale, "The Limiting Driving Force Approach to Ice Loads," in 16th Annual Offshore Technology Conference, Houston, 1984.

[22] H. Deeb, R. Ali Mehdi and A. Hahn, "A review of damage assessment models in the maritime domain," Ships and Offshore Structures, vol. 12, no. S1, pp. S31-S54, 2017.

[23] V. U. Minorsky, "An analysis of ship collisions with reference to protection of nuclear power plants," Sharp (George G.) Inc., New York, 1958.

[24] S. Zhang and P. T. Pedersen, "A method for ship collision damage and energy absorption analysis and its validation," Ships and Offshore Structures, vol. 12, no. S1, pp. S11-S20, 2017.

[25] S. Zhang, "The Mechanics of Ship Collisions," Technical University of Denmark, Lyngby, 1999.

[26] M. A. G. Calle and M. Alves, "A review-analysis on material failure modeling in ship collision," Ocean Engineering, vol. 106, pp. 20-38, 2015.

[27] B. Wang, H.-C. Yu and R. Basu, "Ship and Ice Collision Modeling and Strength Evaluation of LNG Ship Structure," in 27th International Conference on Offshore Mechanics and Arctic Engineering, Estoril, 2008. 
[28] Z. Liu, J. Amdahl and S. Løset, "Numerical simulation of collisions between ships and icebergs," in 20th International Conference on Port and Ocean Engineering under Arctic Conditions, Luleå, 2009.

[29] Z. Yu, W. Lu, M. van den Berg, J. Amdahl and S. Løset, "Glacial ice impacts: Part II: Damage assessment and ice-structure interactions in accidental limit states (ALS)," Marine Structures, vol. 75, 2021.

[30] Z. Liu, "Analytical and numerical analysis of iceberg collisions with ship structures," Norwegian University of Science and Technology, Trondheim, 2011.

[31] E. Kim and J. Amdahl, "Review of existing methods for the analysis of the accidental limit state due to ice actions," in 6th International Conference on Collision and Grounding of Ships and Offshore Structures, Trondheim, 2013.

[32] B. Liu, "Analytical method to assess double-hull ship structures subjected to bulbous bow collision," Ocean Engineering, vol. 142, pp. 27-38, 2017.

[33] B. Sun, Z. Hu and G. Wang, "An analytical method for predicting the ship side structure response in raked bow collisions," Marine Structures, vol. 41, pp. 288311, 2015.

[34] Z. Gao, Z. Hu, G. Wang and Z. Jiang, "An analytical method of predicting the response of FPSO side structures to head-on collision," Ocean Engineering, vol. 87, pp. 121-135, 2014. 
[35] Z. Yu, J. Amdahl and Y. Sha, "Large inelastic deformation resistance of stiffened panels subjected to lateral loading," Marine Structures, vol. 59, pp. 342-367, 2018.

[36] Z. Liu and J. Amdahl, "A new formulation of the impact mechanics of ship collisions and its applications to a ship-iceberg collision," Marine Structures, vol. 23, pp. 360-384, 2010.

[37] J. Amdahl, "Impact from ice floes and icebergs on ships and offshore structures in Polar Regions," IOP Conference Series: Materials Science and Engineering, vol. $700,2019$.

[38] C. Daley and H. Kim, "Ice Collision Forces Considering Structural Deformation," in 29th International Conference on Ocean, Offshore, and Arctic Engineering, Shanghai, 2010.

[39] S. Haris and J. Amdahl, "An analytical model to assess a ship side during a collision," Ships and Offshore Structures, vol. 7, no. 4, pp. 431-448, 2012.

[40] S. Ehlers and K. Tabri, "A combined numerical and semi-analytical collision damage assessment procedure," Marine Structures, vol. 28, no. 1, pp. 101-119, 2012.

[41] C. J. Sun, J. H. Zhou and W. Wu, "Study on Collision of Ship Structure by Simplified Plastic Analysis Method," in 2017 International Conference on Structural, Mechanical and Materials Engineering, Seoul, 2017. 
[42] F. DeBord, T. McAllister, C. Cleary, J. Dolny and R. Kawamoto, "Design Considerations for Operation of Coast Guard Cutters and Combatants in the Arctic," Society of Naval Architects and Marine Engineers, Providence, 2015.

[43] C. G. Daley, A. Kendrick and E. Appolonov, "Plating and Framing Design in the Unified Requirements for Polar Class Ships," in 16th International Conference on Port and Ocean Engineering under Arctic Conditions, Ottawa, 2001.

[44] S. Idrissova, M. Bergström, S. E. Hirdaris and P. Kujala, "Analysis of a CollisionEnergy-Based Method for the Prediction of Ice Loading on Ships," Applied Science, vol. 9, no. 21, 2019.

[45] C. G. Daley, "Derivation of plastic framing requirements for polar ships," Marine Structures, vol. 15, pp. 543-559, 2002.

[46] P. Varsta, I. V. Droumev and M. Hakala, "On Plastic Design of an Ice-Strengthened Frame (Report No 27)," Winter Navigation Research Board, Espoo, 1978.

[47] J. Abraham, "Plastic Response of Ship Structure Subject to Ice Loading," Memorial University of Newfoundland, St. John's, 2008.

[48] L. Hong and J. Amdahl, "Plastic design of laterally patch loaded plated for ships," Marine Structures, vol. 20, pp. 124-142, 2007.

[49] E. Appolonov, "IACS Unified Requirements for Polar Ships Background Notes to Shell Plating Thickness," IACS Ad-Hoc Group on Polar Class Ships, 2000. 
[50] C. G. Daley, "Application of plastic framing requirements for polar ships," Marine Structures, vol. 15, pp. 533-542, 2002.

[51] J. R. Dolny, "A Technical Methodology for Establishing Structural Limitations of Ships in Pack Ice," Memorial University of Newfoundland, St. John's, 2017.

[52] R. G. Budynas and A. M. Sadegh, "Beams: Flexure of Straight Bars," in Roark's Formulas for Stress and Strain, 9th ed., McGraw-Hill Education, 2020.

[53] C. Daley and G. Hermanski, "Investigation of Plastic Limit States for Design of Ship Hull Structures: Ship Frame Research Program - A Experimental Study of Ship Frames and Grillages Subjected to Patch Loads (SSC-457)," Ship Structure Committee, St. John's, 2009.

[54] E. Qi and W. Cui, "Analytical method for ultimate strength calculations of intact and damaged ship hulls," Ships and Offshore Structures, vol. 1, no. 2, pp. 153-163, 2006.

[55] O. Hughes and J. B. Caldwell, "Plate Bending," in Ship Structural Analysis and Design, O. F. Hughes and J. K. Paik, Eds., Jersey City, The Society of Naval Architects and Marine Engineers, 2010, pp. 9-1 to 9-41.

[56] K. P. Ratzlaff and D. J. L. Kennedy, "Analysis of continuous steel plates subjected to uniform transverse loads," Canadian Journal of Civil Engineering, vol. 12, pp. 685-699, 1985. 
[57] S. Timoshenko and S. Woinowsky-Krieger, Theory of plates and shells, 2nd ed., Montreal: McGraw-Hill, 1987.

[58] K.-T. Fang, D. K. Lin, P. Winker and Y. Zhang, "Uniform Design: Theory and Application," Technometrics, vol. 42, no. 3, pp. 237-248, 2000.

[59] D. C. Montgomery, Design and Analysis of Experiments, 8th ed., John Wiley \& Sons, Inc., 2013, pp. 478-553.

[60] K. Yeung, "Uniform Design Based on CD2 Discrepancy -- Un(qs)," 14102004. [Online]. Available: http://www.math.hkbu.edu.hk/UniformDesign/. [Accessed April 2020].

[61] Stat-Ease, Design-Expert 11, Minneapolis, MN: Stat-Ease, Inc., 2018.

[62] R. E. Gagnon and A. Derradji-Aouat, "First Results of Numerical Simulations of Bergy Bit Collisions with the CCGS Terry Fox Icebreaker," in 18th IAHR International Symposium on Ice, Sapporo, 2006.

[63] Z. Liu, J. Amdahl and S. Løset, "Integrated numerical analysis of an iceberg collision with a foreship structure," Marine Structures, vol. 24, no. 4, pp. 377-395, 2011.

[64] H. Kim, C. Daley and H. Kim, "Evaluation of large structural grillages subjected to ice loads in experimental and numerical analysis," Marine Structures, vol. 61, pp. 467-502, 2018. 
[65] Z. Liu, J. Amdahl and S. Løset, "Plasticity based material modelling of ice and its application to ship-iceberg impacts," Cold Regions Science and Technology, vol. 65, no. 3, pp. 326-334, 2011.

[66] D. Han, H. Lee, J. Choung, H. Kim and C. Daley, "Cone ice crushing tests and simulations associated with various yield and fracture criteria," Ships and Offshore Structures, vol. 12, no. S1, pp. S88-S99, 2017.

[67] P. T. Pedersen and S. Zhang, "On impact mechanics in ship collisions," Marine Structures, vol. 11, pp. 429-449, 1998. 
Appendices 


\section{Appendix A - Algorithm (MATLAB Script)}

\section{Contents}

- Inputs

- Step \#1 Find the total kinetic energy of the impact

- $\quad$ Step \#1a Set up the while loop to iterate through possible contact forces

- $\quad$ Step \#2 Determine the ice response: the normal impact area \& the energy used in ice-crushing

- $\quad$ Step \#3a Find the beam deformation energy

- $\quad$ Step \#3b Find the plate deformation energy

- Step \#4 Compare total energy to the ice-crushing and structural response energies

- Display

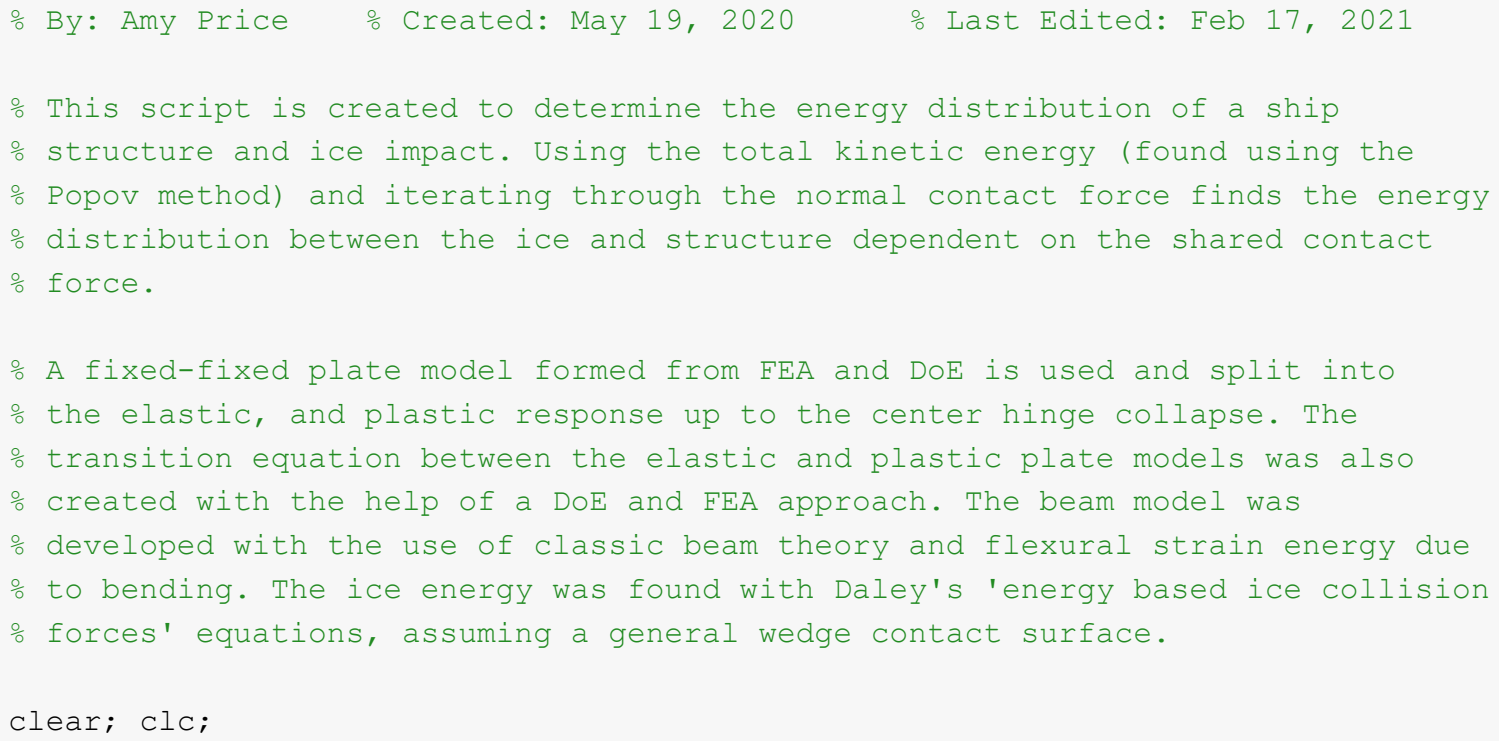

\section{Inputs}

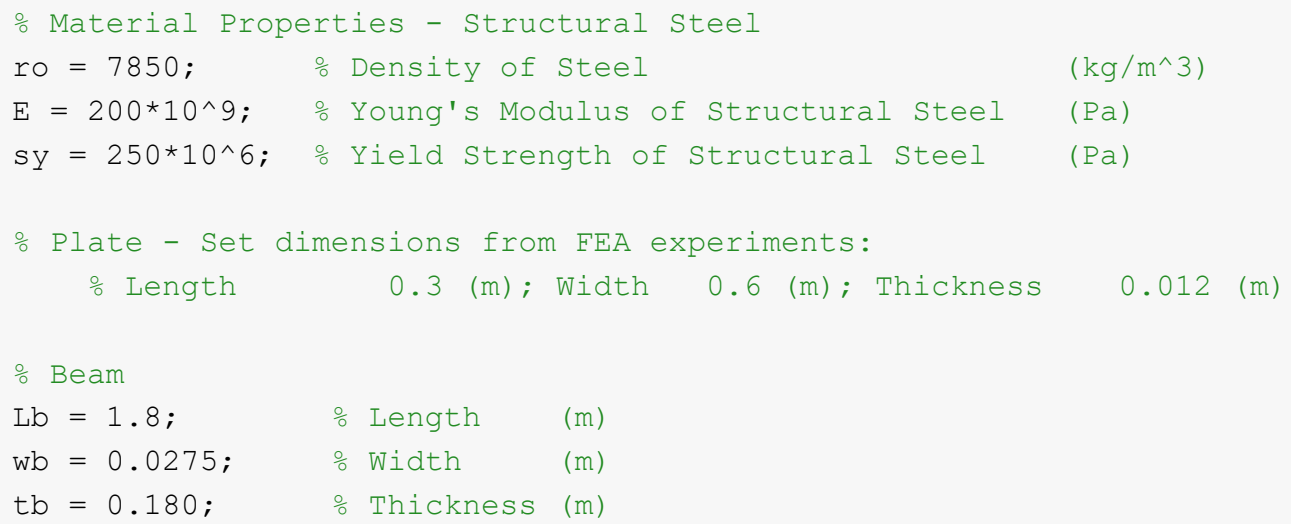




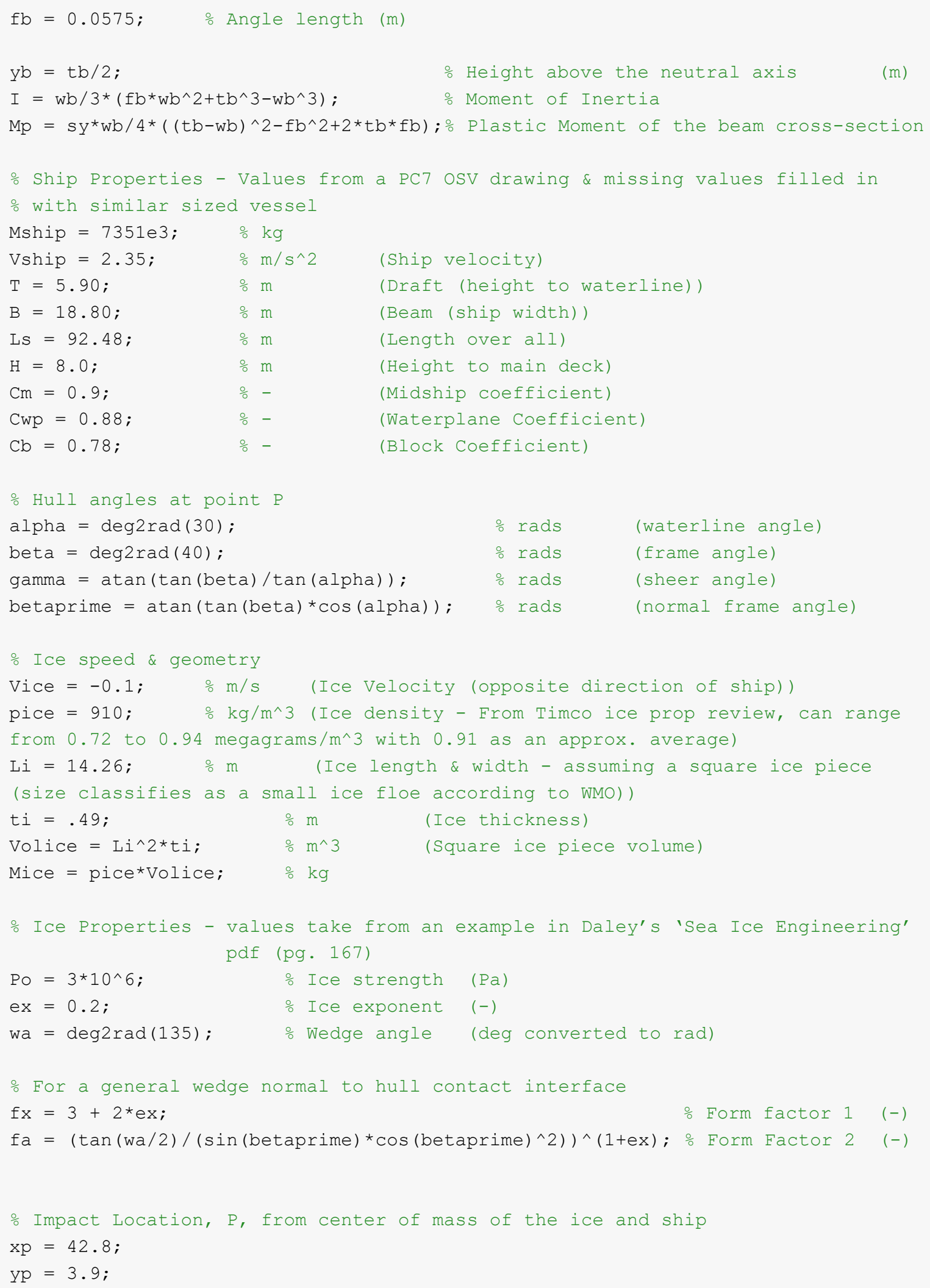




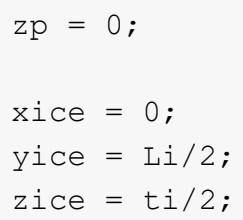

\section{Step \#1 Find the total kinetic energy of the impact}

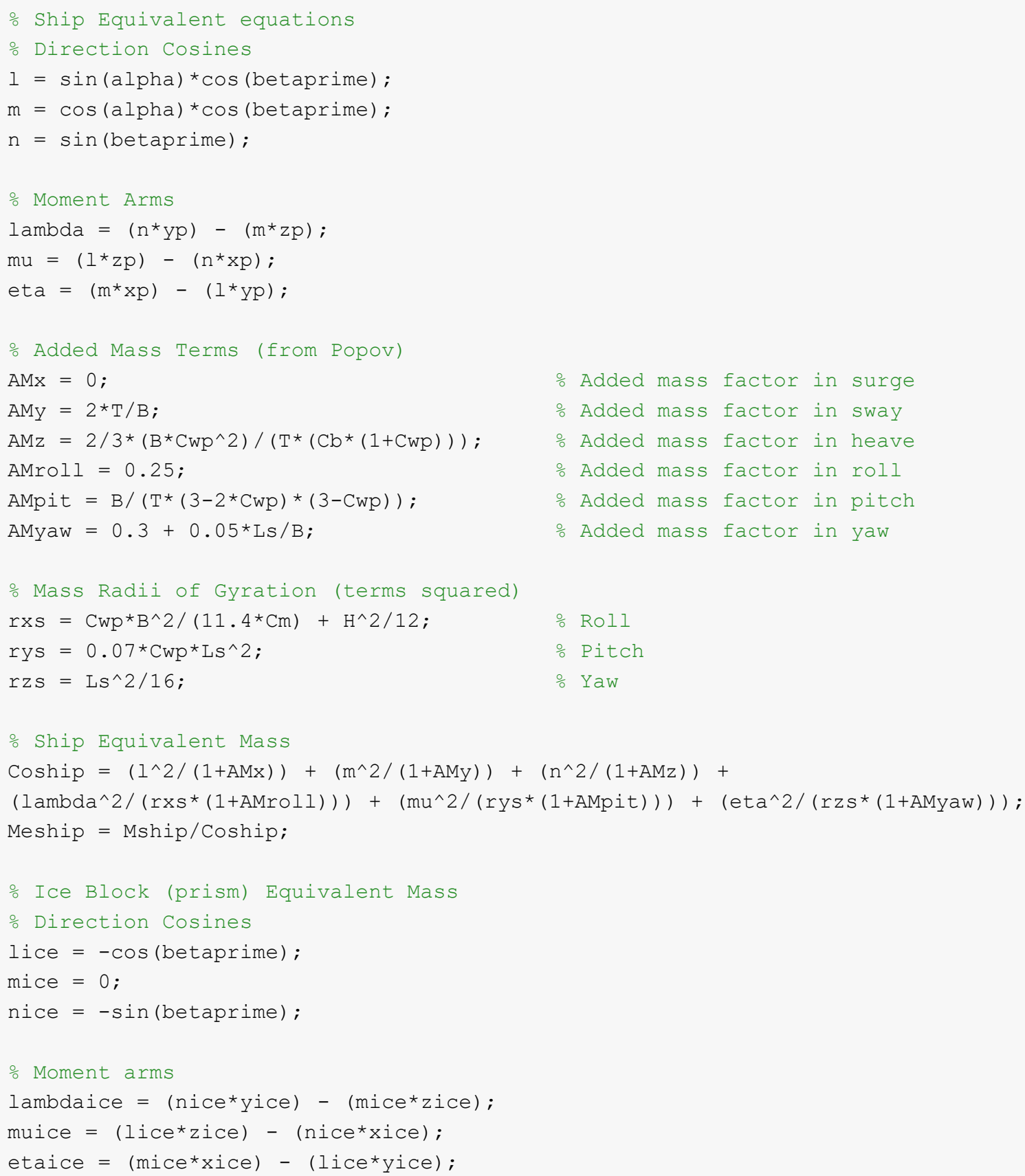




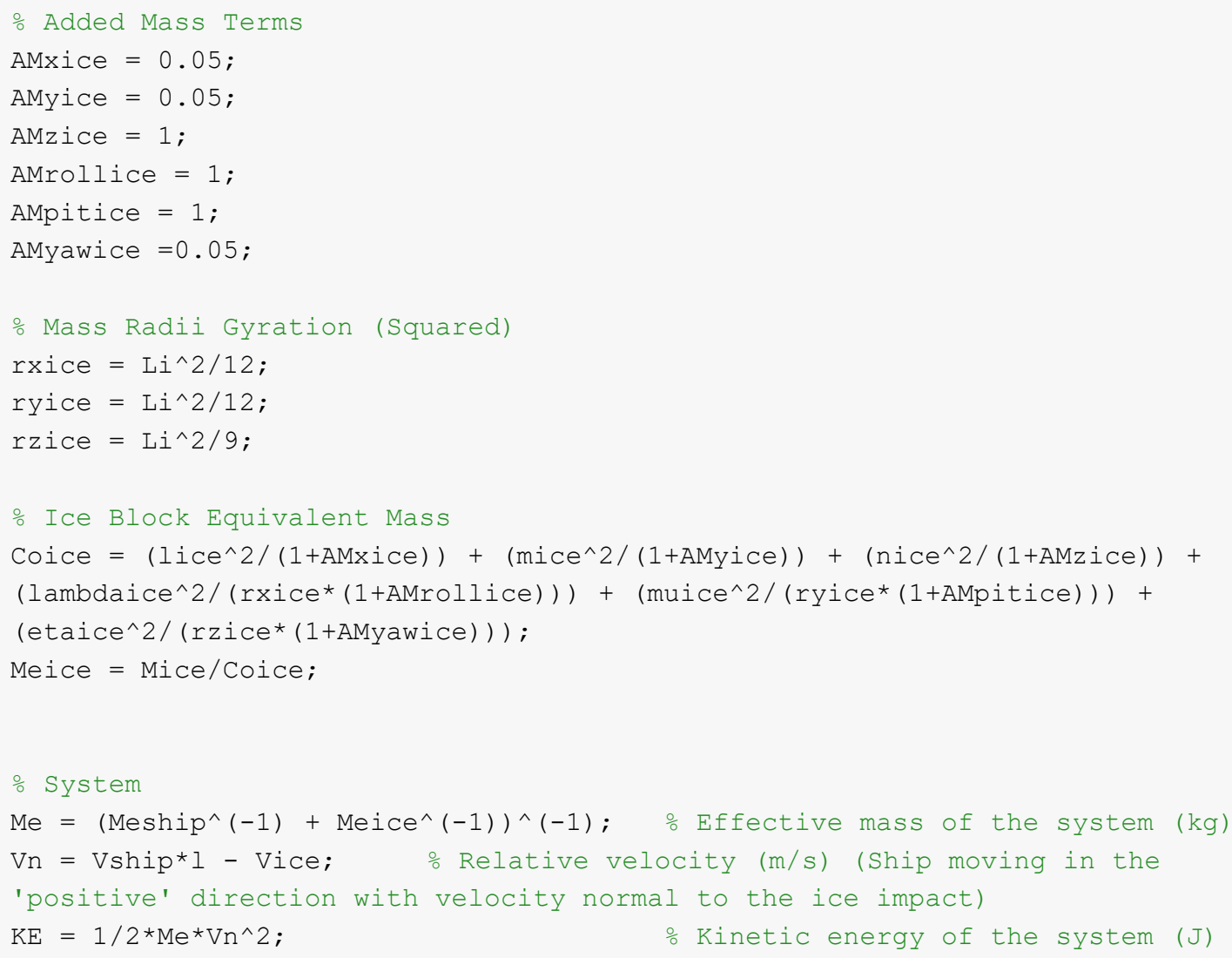

Step \#1a Set up the while loop to iterating through possible contact forces

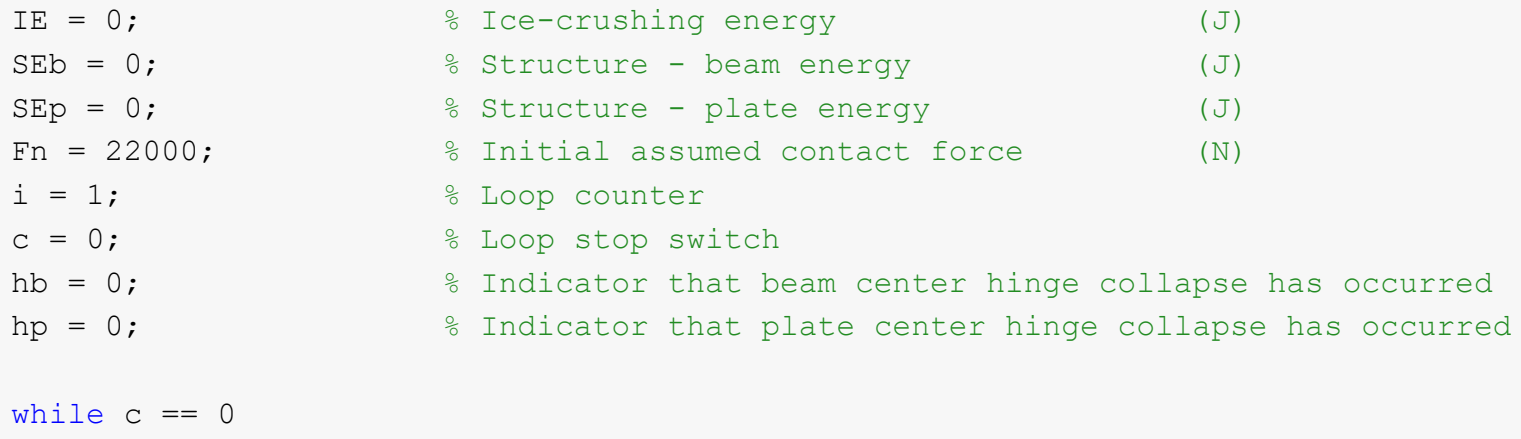

Step \#2 Determine the ice response: the normal impact area \& the energy used in ice-crushing

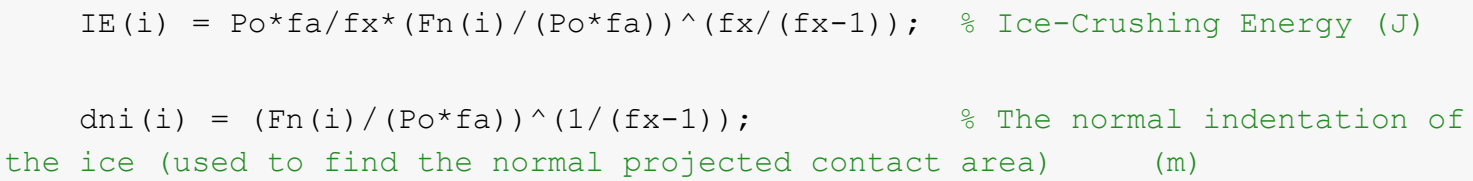




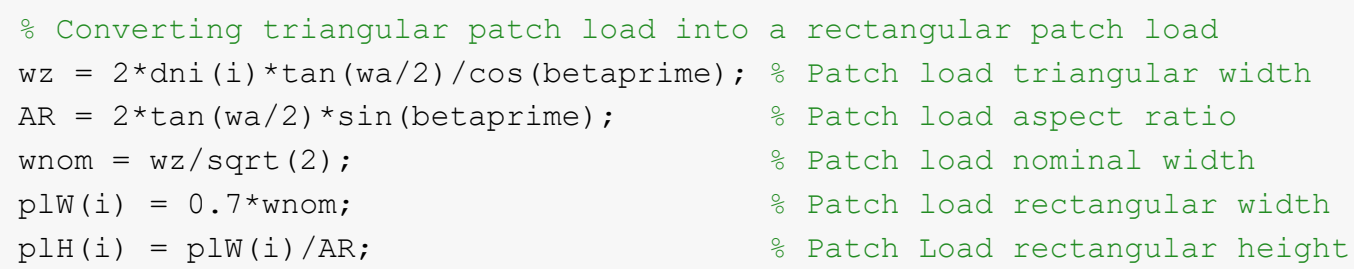

\section{Step \#3a Find the beam deformation energy}

o Checking patch load width is not longer than the beam length if $\mathrm{plW}(\mathrm{i})>\mathrm{L} \mathrm{b}$

$$
\mathrm{b}=\mathrm{Lb} \text {; }
$$

else

$$
\mathrm{b}=\mathrm{plW}(\mathrm{i}) ;
$$

end

$\mathrm{a}=(\mathrm{Lb} / 2)-(\mathrm{b} / 2) ; \quad$ Distance to centred load patch $(\mathrm{m})$

$u=F n(i) / b ; \quad \quad \%$ Uniform distributed load from contact force $(\mathrm{N} / \mathrm{m})$

Reaction Force A for fixed-slider beam (in elastic range)

$\mathrm{RAe}=-\mathrm{u} /\left(2 * L \mathrm{~L}^{\wedge} 3\right) *\left((\mathrm{Lb}-\mathrm{a})^{\wedge} 4-(\mathrm{Lb}-\mathrm{a}-\mathrm{b})^{\wedge} 4\right)-u / L b^{\wedge} 2 *\left((L b-a-b)^{\wedge} 3-(L b-a)^{\wedge} 3\right)$;

\% Reaction Moment A (edge moment) for fixed-slider beam (in elastic range) MAe $=-\left(u /(3 * L b) *\left((L b-a-b)^{\wedge} 3-(L b-a)^{\wedge} 3\right)\right)-\left(u /\left(4 * L b^{\wedge} 2\right) *\left((L b-a)^{\wedge} 4-(L b-a-b)^{\wedge} 4\right)\right)$;

\% Reaction Force A for a pinned patch load (in plastic range)

$\mathrm{RAph}=\mathrm{u} * \mathrm{~b} / \mathrm{L} \mathrm{b} *(\mathrm{~L} \mathrm{~b}-\mathrm{a}-\mathrm{b} / 2)$;

\% Center moment, indicating the center hinge formation (in plastic range) $\mathrm{MC}=\mathrm{RAph} * \mathrm{Lb} / 2-\mathrm{u} / 2 *(\mathrm{Lb} / 2-\mathrm{a})^{\wedge} 2-\mathrm{Mp}$;

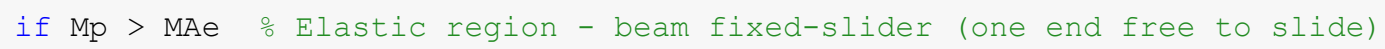

$$
\begin{aligned}
& \circ \text { Flexure Energy }(J) \\
& \mathrm{SEb}(i)=\left(\mathrm { a } ^ { * } \left(\left(\mathrm{u}^{*}\left((\mathrm{a}-\mathrm{Lb}+\mathrm{b})^{\wedge} 4-(\mathrm{Lb}-\mathrm{a})^{\wedge} 4\right)\right) /\left(4^{*} \mathrm{~L} \mathrm{~b}^{\wedge} 2\right)+\left(\mathrm{u}^{*}((\mathrm{a}-\mathrm{Lb}+\right.\right.\right.
\end{aligned}
$$

$\left.\left.\left.b)^{\wedge} 3+(L b-a)^{\wedge} 3\right)\right) /\left(3^{\star} L b\right)\right)^{\wedge} 2+\left(a^{\wedge} 3^{\star}\left(\left(u^{\star}\left((a-L b+b)^{\wedge} 4-(L b-a)^{\wedge} 4\right)\right) /\left(2^{\star} L b^{\wedge} 3\right)\right.\right.$

$\left.\left.+\left(u^{\star}\left((a-L b+b)^{\wedge} 3+(L b-a)^{\wedge} 3\right)\right) / L b^{\wedge} 2\right)^{\wedge} 2\right) / 3+\left(b^{\wedge} 5 * u^{\wedge} 2\right) / 20-a^{\wedge} 2^{\star}\left(\left(u^{\star}((a-L b\right.\right.$

$\left.\left.\left.+b)^{\wedge} 4-(L b-a)^{\wedge} 4\right)\right) /\left(2 * L b^{\wedge} 3\right)+\left(u^{\star}\left((a-L b+b)^{\wedge} 3+(L b-a)^{\wedge} 3\right)\right) / L b^{\wedge} 2\right)^{\star}\left(\left(u^{\star}((a\right.\right.$

$\left.\left.\left.-\mathrm{Lb}+\mathrm{b})^{\wedge} 4-(\mathrm{Lb}-\mathrm{a})^{\wedge} 4\right)\right) /\left(4^{\star} \mathrm{L} \mathrm{b}^{\wedge} 2\right)+\left(\mathrm{u}^{\star}\left((\mathrm{a}-\mathrm{Lb}+\mathrm{b})^{\wedge} 3+(\mathrm{Lb}-\mathrm{a})^{\wedge} 3\right)\right) /\left(3{ }^{\star} \mathrm{Lb}\right)\right)$

$-\left(2 * b^{\wedge} 6 * u^{\wedge} 2\right) /(9 * L b)+\left(11 * b^{\wedge} 7 * u^{\wedge} 2\right) /\left(18 * L b^{\wedge} 2\right)-\left(25 * b^{\wedge} 8 * u^{\wedge} 2\right) /\left(24 * L b^{\wedge} 3\right)+$ $\left(47 * b^{\wedge} 9 * u^{\wedge} 2\right) /\left(48 * \mathrm{~L} b^{\wedge} 4\right)-\left(11 * b^{\wedge} 10 * u^{\wedge} 2\right) /\left(24 * \mathrm{Lb} b^{\wedge} 5\right)+\left(b^{\wedge} 11 * u^{\wedge} 2\right) /\left(12 * L b^{\wedge} 6\right)-$ $\left(2 * a * b^{\wedge} 5 * u^{\wedge} 2\right) /(3 * \mathrm{Lb})+\left(43 * a * b^{\wedge} 6 * u^{\wedge} 2\right) /\left(12 * \mathrm{Lb} b^{\wedge} 2\right)-\left(8 * a * b^{\wedge} 7 * u^{\wedge} 2\right) / L b^{\wedge} 3+$ $\left(35 * a * b^{\wedge} 8 * u^{\wedge} 2\right) /\left(4 * \mathrm{~L} b^{\wedge} 4\right)-\left(55^{*} a^{*} b^{\wedge} 9 * u^{\wedge} 2\right) /\left(12 * L b^{\wedge} 5\right)+\left(11 * a * b^{\wedge} 10 * u^{\wedge} 2\right) /\left(12 * L b^{\wedge} 6\right)-$ $\left(b^{\wedge} 2 * u^{\wedge} 2 *\left(720 * a^{\wedge} 5+2088 * a \wedge 4 * b+2520 * a \wedge 3 * b^{\wedge} 2+1596 * a^{\wedge} 2 * b^{\wedge} 3+532 * a * b^{\wedge} 4+\right.\right.$ $\left.\left.76 * b^{\wedge} 5\right)\right) /\left(144^{\star} \mathrm{Lb} b^{\wedge} 2\right)+\left(b^{\wedge} 2 * u^{\wedge} 2 *\left(144 * a^{\wedge} 4+288 * a^{\wedge} 3 * b+240 * a \wedge 2 * b^{\wedge} 2+96 * a * b^{\wedge} 3+\right.\right.$ $\left.\left.16^{\star} \mathrm{b}^{\wedge} 4\right)\right) /\left(144^{*} \mathrm{Lb}\right)-\left(2 * a^{\wedge} 2^{\star} \mathrm{b}^{\wedge} 4^{\star} \mathrm{u}^{\wedge} 2\right) /\left(3^{\star} \mathrm{Lb}\right)+\left(101^{\star} \mathrm{a}^{\wedge} 2^{\star} \mathrm{b}^{\wedge} 5^{\star} \mathrm{u}^{\wedge} 2\right) /\left(12^{\star} \mathrm{Lb} \mathrm{b}^{\wedge} 2\right)+$ $\left(28 * a^{\wedge} 3 * b^{\wedge} 4 * u^{\wedge} 2\right) /\left(3 * \mathrm{~L} b^{\wedge} 2\right)+\left(4 * a^{\wedge} 4 * b^{\wedge} 3 * u^{\wedge} 2\right) / L b^{\wedge} 2-\left(311^{*} a^{\wedge} 2^{*} b^{\wedge} 6^{\star} u^{\wedge} 2\right) /\left(12 * L b^{\wedge} 3\right)-$ $\left(89 * a^{\wedge} 3 * b^{\wedge} 5 * u^{\wedge} 2\right) /\left(2 * \mathrm{Lb} b^{\wedge} 3\right)-\left(122 * a^{\wedge} 4 * b^{\wedge} 4 * u^{\wedge} 2\right) /\left(3 * \mathrm{Lb} b^{\wedge} 3\right)-\left(16 * a^{\wedge} 5^{\star} b^{\wedge} 3 * u^{\wedge} 2\right) / L b^{\wedge} 3+$ $\left(205 * a \wedge 2 * b^{\wedge} 7 * u^{\wedge} 2\right) /\left(6 * L b^{\wedge} 4\right)+\left(449 * a \wedge 3 * b^{\wedge} 6 * u^{\wedge} 2\right) /\left(6 * L b^{\wedge} 4\right)+$ 


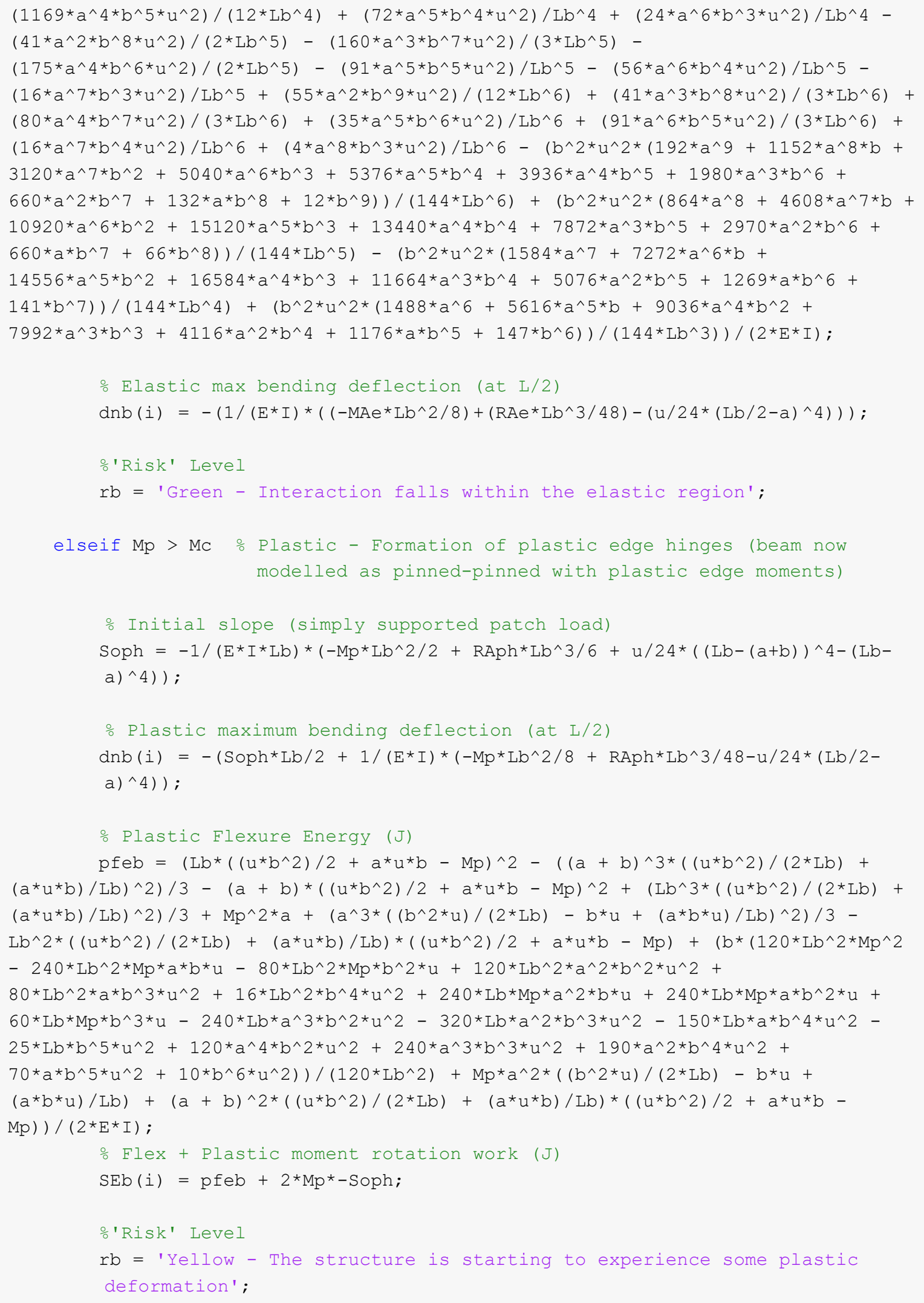




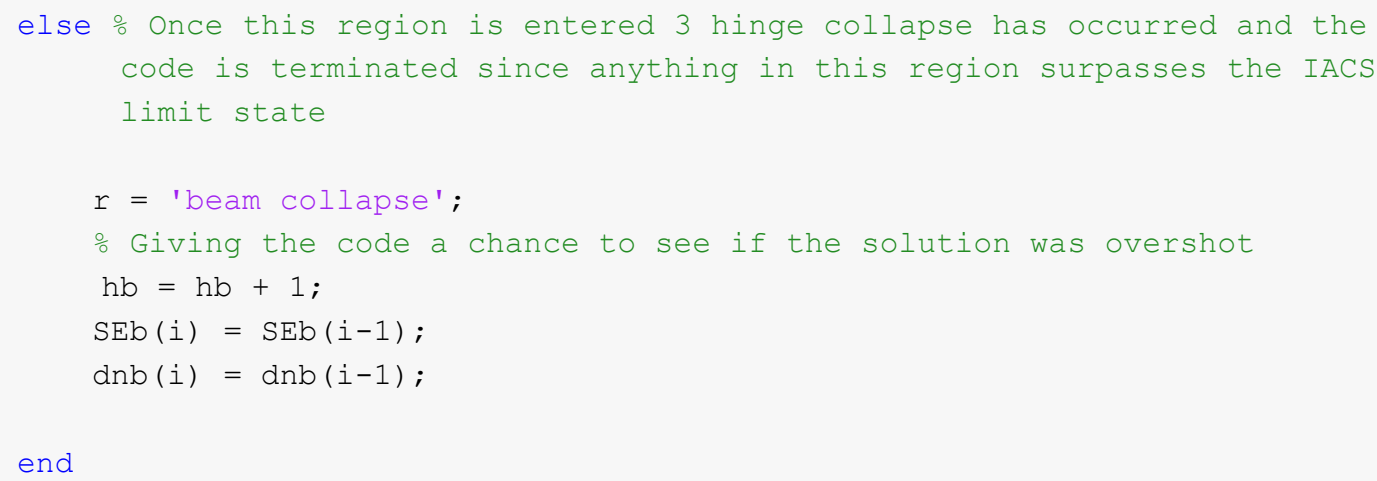

\section{Step \#3b Find the plate deformation energy}

\% Checking that the patch load height falls within the meta-model's patch load height range 


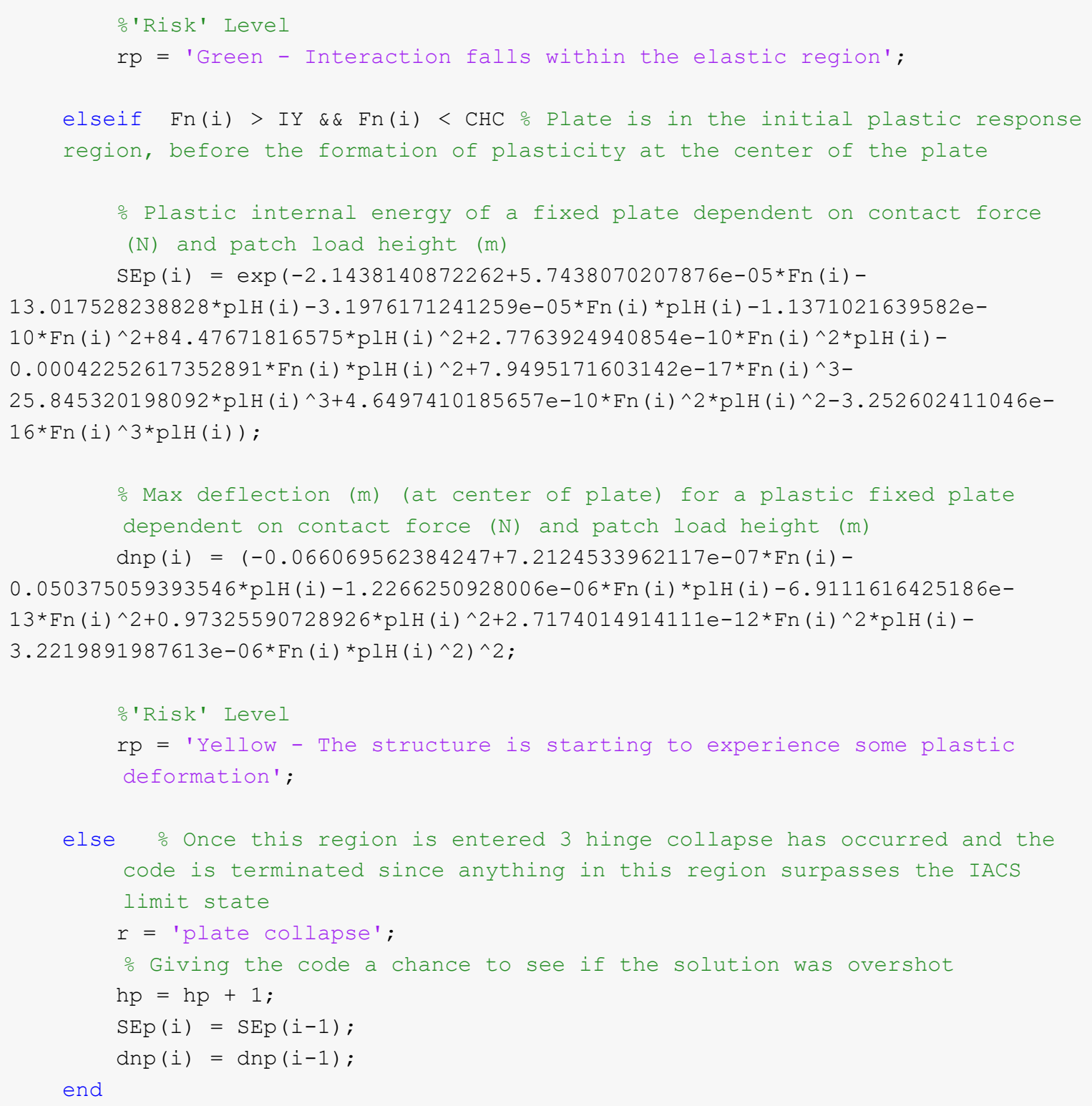

\section{Step \#4 Compare total energy to the ice-crushing and structural response energies}

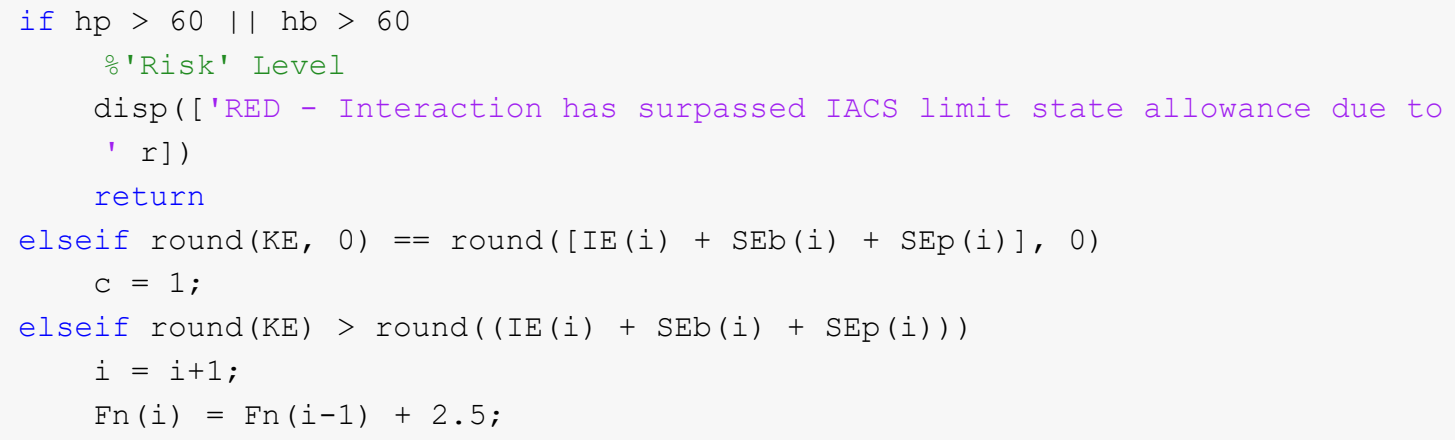


else

$i=i+1 ;$

Fn $(i)=$ Fn $(i-1)-0.05 ;$ smaller since narrowing down at this point \& checking if the solution has been skipped

end

end

\section{Display}

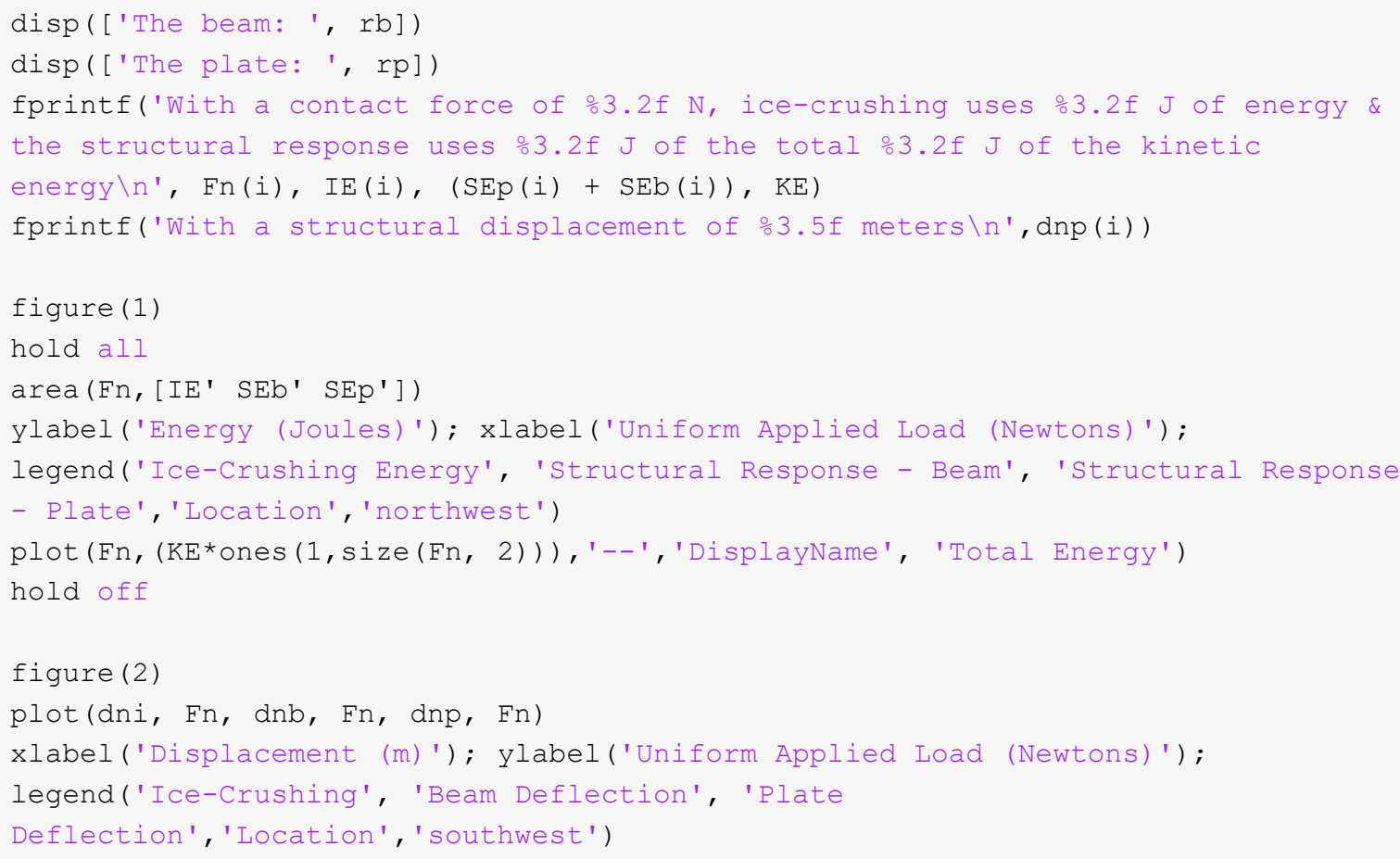

The beam: Yellow - The structure is starting to experience some plastic deformation

The plate: Yellow - The structure is starting to experience some plastic deformation

With a contact force of $357412.50 \mathrm{~N}$, ice-crushing uses $17293.73 \mathrm{~J}$ of energy \& the structural response uses $927.89 \mathrm{~J}$ of the total $18221.65 \mathrm{~J}$ of the kinetic energy

With a structural displacement of 0.00547 meters 


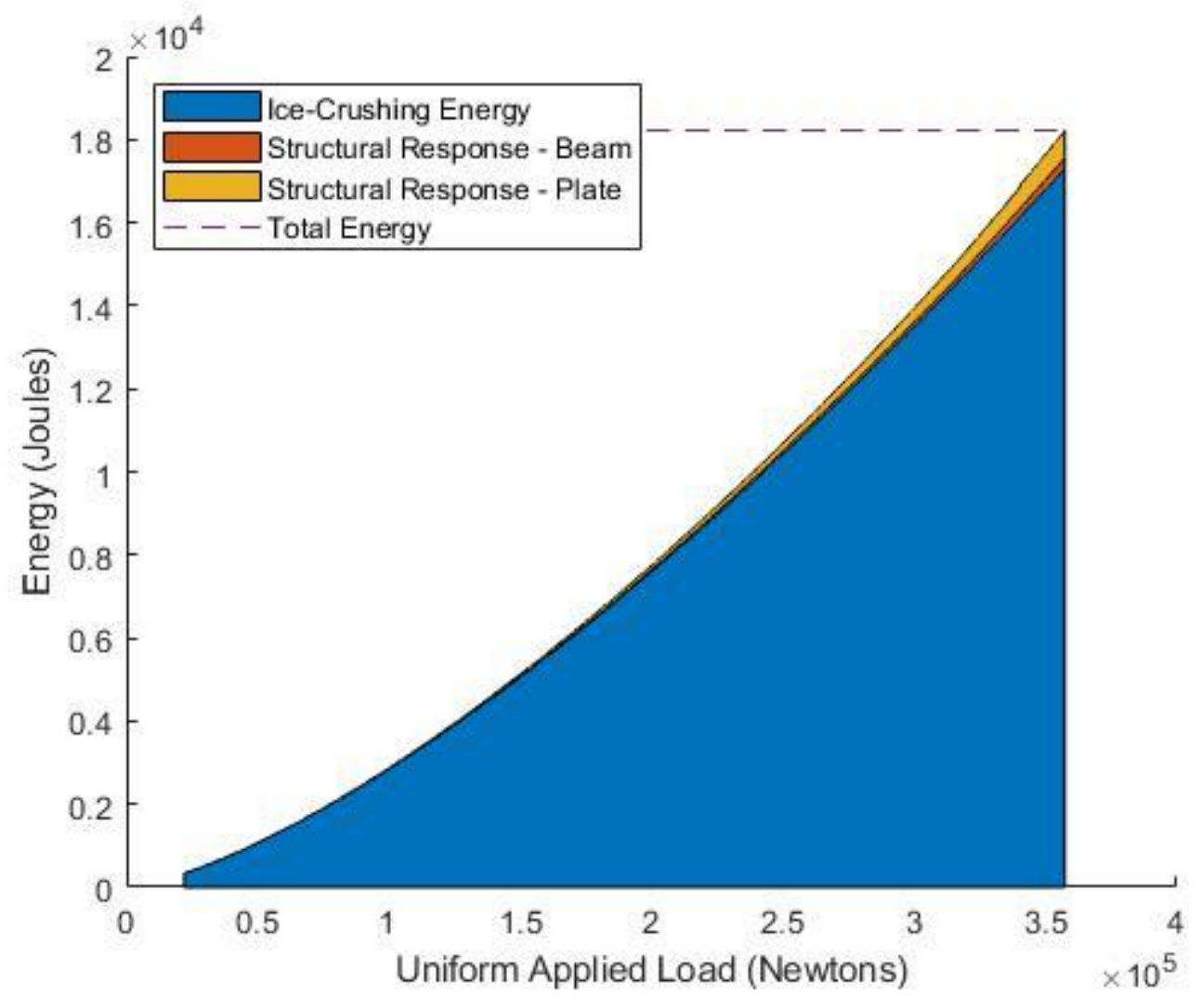




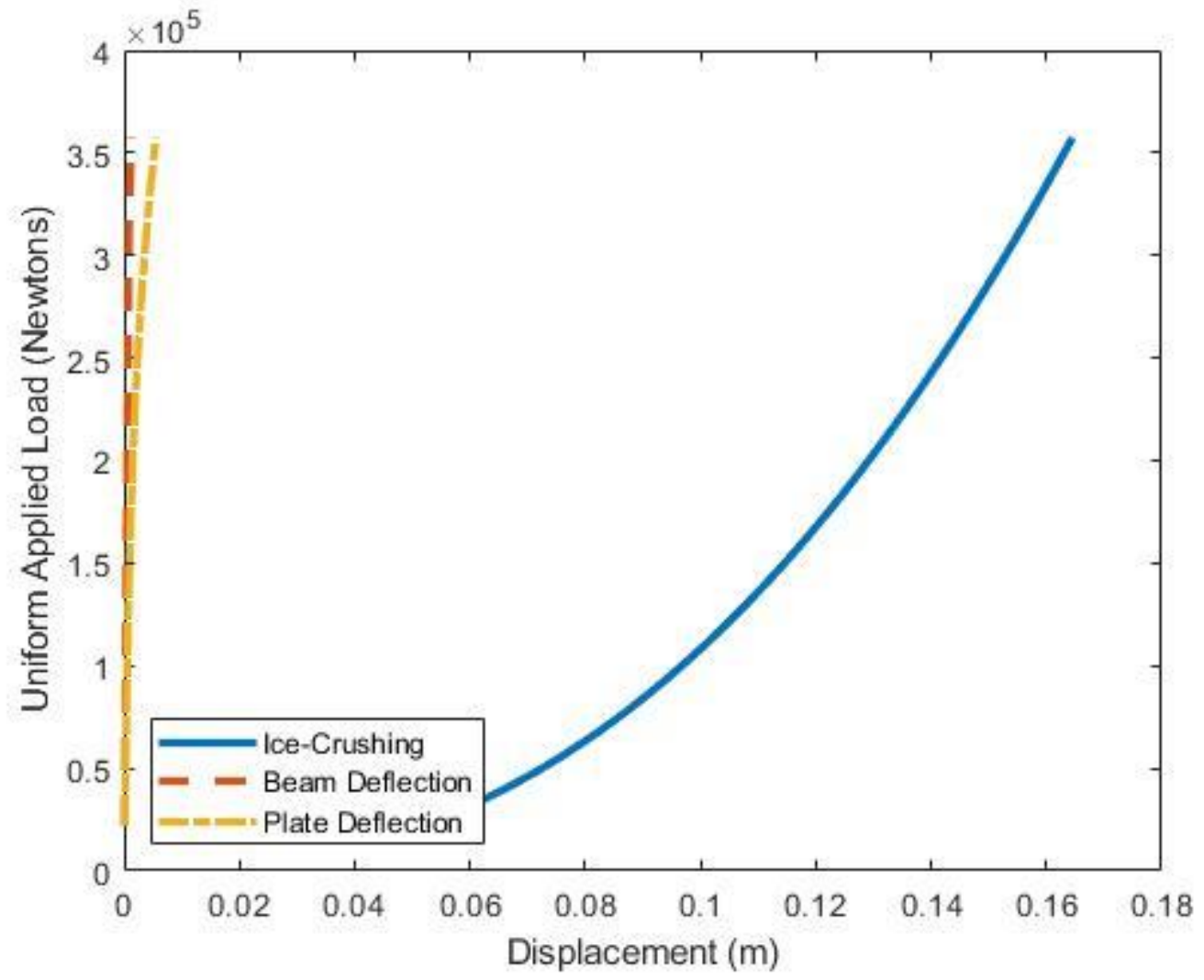

Published with MATLAB® R2019b 


\section{Appendix B - Popov Model Description}

A Popov collision summarizes the complex six-degree of freedom of a two-body collision down to a single-degree of freedom, through an effective mass term. For the Popov collision model implemented into this algorithm, effective mass terms for both the ship and ice are determined and then applied to find an effective mass of the whole collision system, as presented in Section 3.2. In this appendix, the additional inertial functions used to determine Eq. 5 are presented.

The effective mass, $M_{e}$, can be considered a point mass at the point ' $P$ ' of the contact force, $F_{n}$, along the normal vector with the magnitude $\ddot{\zeta}$, Eq. i. The coordinates of point ' $P$ ' are relative to the center of mass of the ship or ice block. The effective mass, Eq. ii, is a function of the inertial properties, mass, radii of gyration, and moment arms, summarized in a coefficient, $C_{o}$, and linearly proportional to the mass of the body, $M$.

$$
\begin{aligned}
& M_{e}=\frac{F_{n}}{\ddot{\zeta}} \\
& M_{e}=\frac{M}{C_{o}}
\end{aligned}
$$

Eq. ii

\section{Ship Effective Mass Coefficient}

To determine the effective mass coefficient of the ship (Eq. iii) the following hull terms, directional cosines, moment arms, mass radii of gyration, and added mass terms are listed as Eq. iv through Eq. xx. The hull angle and directional cosine terms (Eq. vi to Eq. viii) are dependent on the waterline angel, $\alpha$, frame angel, $\beta$, normal frame angle, $\beta$ ', and sheer 
angle, $\gamma$, at the impact location of point ' $P$ '. The $x, y, z$ terms of the moment arms (Eq. ix to Eq. xi) are the location of the impact relative to the ship's center of mass. Lastly the radii of gyration (Eq. xviii to Eq. $\mathrm{xx}$ ) and added mass terms (Eq. xii to Eq. xvii) are dependent on the ship's general dimensions such as draft, $T$, breadth, $B_{\text {ship }}$, waterplane coefficient, $C_{w p}$, block coefficient, $C_{B}$, overall length of the ship, $L_{s h i p}$, height (depth), $H$, and midship coefficient $C_{m}$.

$$
C_{o_{\text {ship }}}=\frac{l^{2}}{1+A M_{x}}+\frac{m^{2}}{1+A M_{y}}+\frac{n^{2}}{1+A M_{z}}+\frac{\lambda^{2}}{r x^{2}\left(1+A M_{\text {roll }}\right)}+\frac{\mu^{2}}{r y^{2}\left(1+A M_{\text {pit }}\right)}+\frac{\eta^{2}}{r z^{2}\left(1+A M_{\text {yaw }}\right)} \quad \text { Eq. iii }
$$

Hull angle relationships:

$$
\begin{array}{lr}
\tan (\beta)=\tan (\alpha) \tan (\gamma) & \text { Eq. iv } \\
\tan \left(\beta^{\prime}\right)=\tan (\beta) \cos (\alpha) & \text { Eq. } v
\end{array}
$$

Directional cosines, based on the ship hull angles:

$$
\begin{array}{lr}
l=\sin (\alpha) \cos \left(\beta^{\prime}\right) & \text { Eq. vi } \\
m=\cos (\alpha) \cos \left(\beta^{\prime}\right) & \text { Eq. vii } \\
n=\sin \left(\beta^{\prime}\right) & \text { Eq. viii }
\end{array}
$$

Moment arms:

$$
\begin{array}{llr}
\lambda=n y_{p}-m z_{p} & \text { (Roll) } & \text { Eq. } \mathrm{ix} \\
\mu=l z_{p}-n x_{p} & \text { (Pitch) } & \text { Eq. } \mathrm{x} \\
\eta=m x_{p}-l y_{p} & \text { (Yaw) } & \text { Eq. xi }
\end{array}
$$

Ship geometric added mass terms (Popov):

$$
A M_{x}=0
$$

Eq. xii 


$$
\begin{array}{lll}
A M_{y}=2 \frac{T}{B_{\text {ship }}} & \text { (Sway) } & \text { Eq. xiii } \\
A M_{z}=\frac{2 B_{\text {ship }} C_{w p}{ }^{2}}{3 T C_{B}\left(1+C_{w p}\right)} & \text { (Heave) } & \text { Eq. xiv } \\
A M_{\text {roll }}=0.25 & \text { (Roll) } & \text { Eq. xv } \\
A M_{\text {pit }}=\frac{B_{\text {ship }}}{T\left(3-2 C_{w p}\right)\left(3-C_{w p}\right)} & \text { (Pitch) } & \text { Eq. xvi } \\
A M_{\text {yaw }}=0.3+0.05 \frac{L_{\text {ship }}}{B_{\text {ship }}} & \text { (Yaw) } & \text { Eq. xvii }
\end{array}
$$

Mass radii of gyration squared:

$$
\begin{aligned}
& r x^{2}=\frac{C_{w p} B_{\text {ship }}^{2}}{11.4 C_{m}}+\frac{H^{2}}{12} \\
& r y^{2}=0.07 C_{w p} L_{\text {ship }}^{2} \\
& r z^{2}=\frac{L_{\text {ship }}^{2}}{16}
\end{aligned}
$$

Eq. xviii

Eq. xix

(Yaw)

Eq. $x x$

\section{Ice Block Effective Mass Coefficient}

Popov originally assumed a circular ice floe, but to more closely model the ice-wedge contact geometry assumed in the IACS Polar rules, Dolny [51] estimated the inertial terms (Eq. xxi to Eq. xxxvi) for the effective mass of a square ice floe (Eq. xxi). In addition to the $x, y, z$ location of the impact relative to the center of mass of the ice square floe, the normal frame angel of the ship at the point of contact, $\beta$, and the edge length of the square ice floe, $L_{i c e}$, are required. 


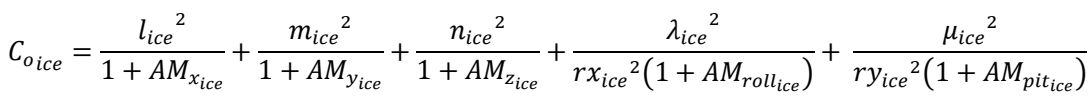

$$
\begin{aligned}
& +\frac{\eta_{i c e}^{2}}{r z_{i c e}{ }^{2}\left(1+A M_{\text {yaw }}\right)}
\end{aligned}
$$

Eq. $x x i$

Ice block directional cosines:

$$
\begin{aligned}
& l_{i c e}=-\cos \left(\beta^{\prime}\right) \\
& m_{i c e}=0 \\
& n_{\text {ice }}=-\sin \left(\beta^{\prime}\right)
\end{aligned}
$$

Eq. xxii

Eq. xxiii

Eq. xxiv

Moment arms:

$$
\begin{aligned}
& \lambda_{i c e}=n_{i c e} y_{p}-m_{i c e} z_{p} \\
& \mu_{i c e}=l_{i c e} z_{p}-n_{i c e} x_{p} \\
& \eta_{i c e}=m_{i c e} x_{p}-l_{i c e} y_{p}
\end{aligned}
$$

Eq. $x x v$

Eq. $x x v i$

Eq. xxvii

Ice block added mass terms (Dolny [51]):
$A M_{x_{i c e}}=0.05$
Eq. xxviii
$A M_{y_{\text {ice }}}=0.05$
(Sway)
Eq. xxix
$A M_{\text {zice }}=1.0$
(Heave)
Eq. $\mathrm{xxx}$
$A M_{\text {roll }}$ ice $=1.0$
(Roll)
Eq. $x x x i$
$A M_{\text {pit }}$ ice $=1.0$
(Pitch)
Eq. xxxii
$A M_{\text {yaw }_{\text {ice }}}=0.05$
(Yaw)
Eq. xxxiii

Mass radii of gyration squared:

$$
r x_{i c e}{ }^{2}=\frac{L_{i c e^{2}}}{12}
$$

Eq. xxxiv 


$$
\begin{aligned}
& r y_{i c e}{ }^{2}=\frac{L_{i c e^{2}}}{12} \\
& r z_{i c e^{2}}=\frac{L_{i c e^{2}}}{9}
\end{aligned}
$$

Eq. $\mathrm{XxXv}$

Eq. xxxvi 


\section{Appendix C - Beam Patch Load Derivation}

To derive the moment across the beam, Figure C.1, classical beam theory is applied and modelled with the Macaulay method. The moment equations are converted into piecewise functions to allow for the integration of the strain energy across the length of the beam, seen in section 3.4.1.

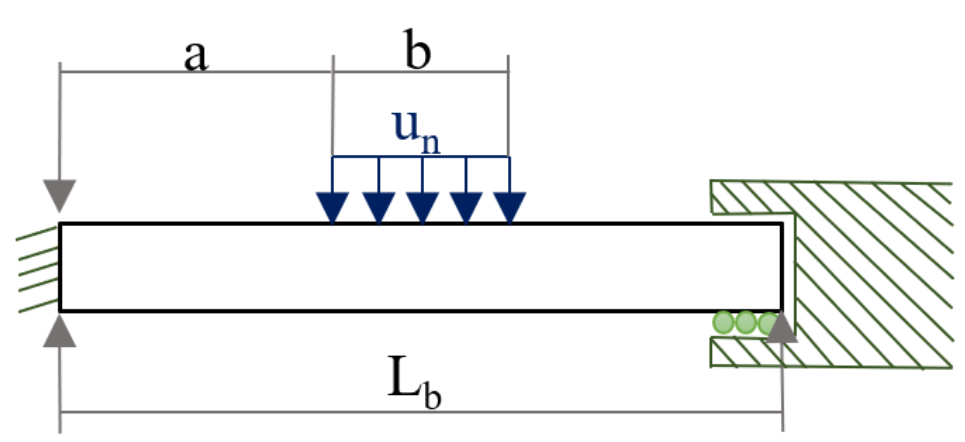

Figure C.1 Beam Boundary and Loading Conditions

\section{Elastic Beam Derivation}

With Figure C.2, the elastic beam responses are derived with Macaulay brackets. First, the reaction forces $(F(x))$ across the beam are determined, then integrated to find the shear force $(Q(x))$, bending moment $(M(x))$, slope $(\theta(x))$, and deflection $(v(x))$ across the length of the beam.

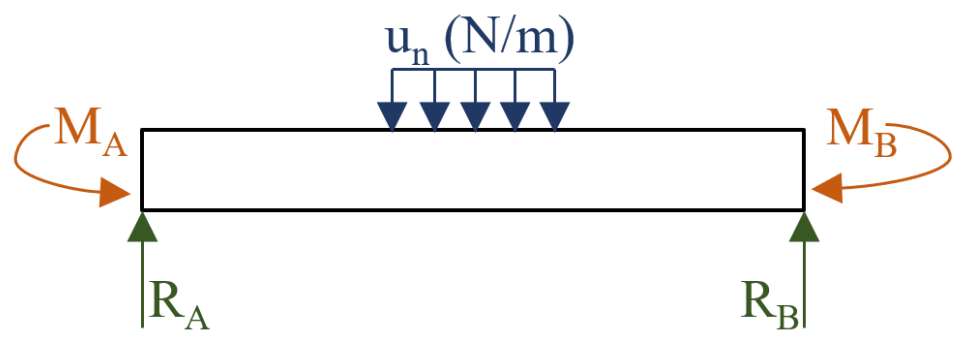

Figure C.2 Elastic Beam Reaction Forces and Moments 
Applied and Reaction Forces [N]

$F(x)=R_{A}\langle x-0\rangle^{-1}-u_{n}\langle x-a\rangle^{0}+u_{n}\langle x-(a+b)\rangle^{0}+R_{B}\left\langle x-L_{b}\right\rangle^{-1}$

Shear Force [N]

$Q(x)=R_{A}\langle x-0\rangle^{0}-u_{n}\langle x-a\rangle^{1}+u_{n}\langle x-(a+b)\rangle^{1}+R_{B}\left\langle x-L_{b}\right\rangle^{0}$

Bending Moment [Nm]

$$
\begin{gathered}
M(x)=-M_{A}\langle x-0\rangle^{0}+R_{A}\langle x-0\rangle^{1}-\frac{u_{n}}{2}\langle x-a\rangle^{2}+\frac{u_{n}}{2}\langle x-(a+b)\rangle^{2}+R_{B}\left\langle x-L_{b}\right\rangle^{1} \\
-M_{B}\left\langle x-L_{b}\right\rangle^{0}
\end{gathered}
$$

Slope of Beam [rad]

$$
\begin{aligned}
\theta(x)=\theta(0)+ & \frac{1}{E I}\left(-M_{A}\langle x-0\rangle^{1}+\frac{R_{A}}{2}\langle x-0\rangle^{2}-\frac{u_{n}}{6}\langle x-a\rangle^{3}+\frac{u_{n}}{6}\langle x-(a+b)\rangle^{3}\right. \\
& \left.+\frac{R_{B}}{2}\left\langle x-L_{b}\right\rangle^{2}-M_{B}\left\langle x-L_{b}\right\rangle^{1}\right)
\end{aligned}
$$

\section{Beam Deflection [m]}

$$
\begin{aligned}
v(x)=v(0)+ & \theta(0) x \\
& +\frac{1}{E I}\left(\frac{-M_{A}}{2}\langle x-0\rangle^{2}+\frac{R_{A}}{6}\langle x-0\rangle^{3}-\frac{u_{n}}{24}\langle x-a\rangle^{4}+\frac{u_{n}}{24}\langle x-(a+b)\rangle^{4}\right. \\
& \left.+\frac{R_{B}}{6}\left\langle x-L_{b}\right\rangle^{3}-\frac{M_{B}}{2}\left\langle x-L_{b}\right\rangle^{2}\right)
\end{aligned}
$$

\section{Determining the Beam Reaction Terms}

Since the elastic boundary conditions are fixed but free-to-pull in, at both ends of the beam $\left(x=0 \& x=L_{b}\right)$ the slope and deflection of the beam are zero. 
$\therefore \theta(0)=0 \& v(0)=0$

To determine $R_{A}$ and $M_{A}$, knowing that the slope and deflection are zero at the ends of the beam, the slope and deflection equations are solved at $x=L_{b}$ and arranged as a system of equations to determine the reaction force and moment at the start $(x=0)$ of the beam.

$$
\begin{aligned}
& \theta\left(L_{b}\right)=0=\frac{1}{E I}\left(-M_{A}\left\langle L_{b}-0\right\rangle^{1}+\frac{R_{A}}{2}\left\langle L_{b}-0\right\rangle^{2}-\frac{u_{n}}{6}\left\langle L_{b}-a\right\rangle^{3}+\frac{u_{n}}{6}\left\langle L_{b}-(a+b)\right\rangle^{3}\right. \\
& \left.+\frac{R_{B}}{2}\left\langle L_{b}-L_{b}\right\rangle^{2}-M_{B}\left\langle L_{b}-L_{b}\right\rangle^{1}\right) \\
& M_{A}=\frac{R_{A}}{2} L_{b}-\frac{u_{n}}{6 L_{b}}\left(L_{b}-a\right)^{3}+\frac{u_{n}}{6 L_{b}}\left(L_{b}-a-b\right)^{3} \\
& v\left(L_{b}\right)=0=\frac{1}{E I}\left(\frac{-M_{A}}{2}\left\langle L_{b}-0\right\rangle^{2}+\frac{R_{A}}{6}\left\langle L_{b}-0\right\rangle^{3}-\frac{u_{n}}{24}\left\langle L_{b}-a\right\rangle^{4}+\frac{u_{n}}{24}\left\langle L_{b}-(a+b)\right\rangle^{4}\right. \\
& \left.+\frac{R_{B}}{6}\left\langle L_{b}-L_{b}\right\rangle^{3}-\frac{M_{B}}{2}\left\langle L_{b}-L_{b}\right\rangle^{2}\right) \\
& R_{A}=\frac{3 M_{A}}{L_{b}}+\frac{u_{n}}{4 L_{b}^{3}}\left(L_{b}-a\right)^{4}-\frac{u_{n}}{4 L_{b}^{3}}\left(L_{b}-a-b\right)^{4}
\end{aligned}
$$

Therefore:

$$
\begin{aligned}
& R_{A}=\frac{u_{n}}{2 L_{b}^{3}}\left[\left(L_{b}-a-b\right)^{4}-\left(L_{b}-a\right)^{4}\right]+\frac{u_{n}}{L_{b}^{2}}\left[\left(L_{b}-a\right)^{3}-\left(L_{b}-a-b\right)^{3}\right] \\
& M_{A}=\frac{u_{n}}{4 L_{b}^{2}}\left[\left(L_{b}-a-b\right)^{4}-\left(L_{b}-a\right)^{4}\right]+\frac{u_{n}}{3 L_{b}}\left[\left(L_{b}-a\right)^{3}-\left(L_{b}-a-b\right)^{3}\right]
\end{aligned}
$$


Now with the reaction force and moment known at the beginning of the beam, the bending moment across the beam can be broken up and modelled with piecewise functions.

$\mathbf{0} \leq \boldsymbol{x} \leq \boldsymbol{a}$

$$
\begin{aligned}
& M_{1}(x)=-M_{A}\langle x-0\rangle^{0}+R_{A}\langle x-0\rangle^{1} \\
& M_{1}(x)=-M_{A}+R_{A} x
\end{aligned}
$$

$\boldsymbol{a} \leq \boldsymbol{x} \leq \boldsymbol{a}+\boldsymbol{b}$

$$
\begin{aligned}
& M_{2}(x)=-M_{A}\langle x-0\rangle^{0}+R_{A}\langle x-0\rangle^{1}-\frac{u_{n}}{2}\langle x-a\rangle^{2} \\
& M_{2}(x)=-M_{A}+R_{A} x-\frac{u_{n}}{2}(x-a)^{2} \\
& \boldsymbol{a}+\boldsymbol{b} \leq \boldsymbol{x} \leq \boldsymbol{L} \\
& M_{3}(x)=-M_{A}\langle x-0\rangle^{0}+R_{A}\langle x-0\rangle^{1}-\frac{u_{n}}{2}\langle x-a\rangle^{2}+\frac{u_{n}}{2}\langle x-(a+b)\rangle^{2} \\
& M_{3}(x)=-M_{A}+R_{A} x-\frac{u_{n}}{2}\left(x^{2}-2 a x+a^{2}\right) \\
& \quad+\frac{u_{n}}{2}\left(x^{2}-2 a x-2 b x+a^{2}+2 a b+b^{2}\right) \\
& M_{3}(x)=-M_{A}+R_{A} x-u_{n} b(x-a-b / 2)
\end{aligned}
$$

\section{Initial Plastic Beam Response Derivation}

Similar to the elastic response, the reaction and applied forces, shear force, bending moment, slope, and deflection across the length of the beam are determined with Macaulay's brackets and the adjusted boundary conditions. A simply-supported beam with 
superimposed plastic moments is used to model the onset of yield at the ends of the beam, shown in Figure C.3.
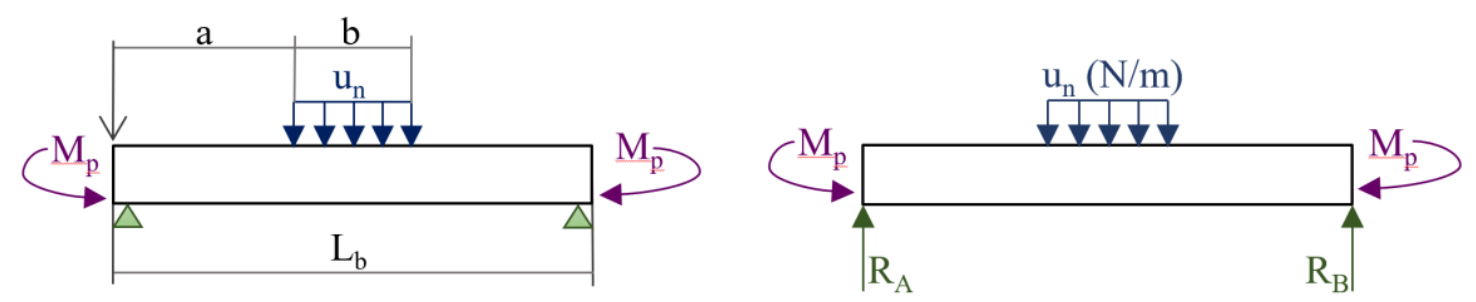

Figure C.3 Plastic Beam Reaction Forces and Moments

Applied and Reaction Forces [N]

$F(x)=R_{A}\langle x-0\rangle^{-1}-u_{n}\langle x-a\rangle^{0}+u_{n}\langle x-(a+b)\rangle^{0}+R_{B}\left\langle x-L_{b}\right\rangle^{-1}$

Shear Force [N]

$Q(x)=R_{A}\langle x-0\rangle^{0}-u_{n}\langle x-a\rangle^{1}+u_{n}\langle x-(a+b)\rangle^{1}+R_{B}\left\langle x-L_{b}\right\rangle^{0}$

\section{Bending Moment [Nm]}

$$
\begin{aligned}
M(x)=-M_{p}\langle x-0\rangle^{0}+R_{A}\langle x-0\rangle^{1}-\frac{u_{n}}{2}\langle x-a\rangle^{2}+\frac{u_{n}}{2}\langle x-(a+b)\rangle^{2}+R_{B}\left\langle x-L_{b}\right\rangle^{1} \\
-M_{p}\left\langle x-L_{b}\right\rangle^{0}
\end{aligned}
$$

\section{Slope of Beam [rad]}

$$
\begin{aligned}
\theta(x)=\theta(0)+ & \frac{1}{E I}\left(-M_{p}\langle x-0\rangle^{1}+\frac{R_{A}}{2}\langle x-0\rangle^{2}-\frac{u_{n}}{6}\langle x-a\rangle^{3}+\frac{u_{n}}{6}\langle x-(a+b)\rangle^{3}\right. \\
& \left.+\frac{R_{B}}{2}\left\langle x-L_{b}\right\rangle^{2}-M_{p}\left\langle x-L_{b}\right\rangle^{1}\right)
\end{aligned}
$$




\section{Beam Deflection [m]}

$$
\begin{aligned}
v(x)=v(0)+ & \theta(0) x \\
& +\frac{1}{E I}\left(\frac{-M_{p}}{2}\langle x-0\rangle^{2}+\frac{R_{A}}{6}\langle x-0\rangle^{3}-\frac{u_{n}}{24}\langle x-a\rangle^{4}+\frac{u_{n}}{24}\langle x-(a+b)\rangle^{4}\right. \\
& \left.+\frac{R_{B}}{6}\left\langle x-L_{b}\right\rangle^{3}-\frac{M_{p}}{2}\left\langle x-L_{b}\right\rangle^{2}\right)
\end{aligned}
$$

\section{Determining the Beam Reaction Terms}

With the plastic boundary conditions now set as pinned with superimposed end moments, at both ends of the beam $\left(x=0 \& x=L_{b}\right)$ the deflection of the beam is zero, and the moment at the ends of the beam is the plastic moment. Therefore the $v(0)$ term is zero. This leaves $R_{A}$ and $\theta(0)$ as unknowns to be solved. First, the reaction force, $R_{A}$, is determined by solving for the moment at $x=L_{b}$.

$$
\begin{aligned}
& M\left(L_{b}\right)=-M_{p}=-M_{p}+R_{A}\left\langle L_{b}-0\right\rangle^{1}-\frac{u_{n}}{2}\left\langle L_{b}-a\right\rangle^{2}+\frac{u_{n}}{2}\left\langle L_{b}-(a+b)\right\rangle^{2} \\
& M\left(L_{b}\right)=0=R_{A} L_{b}-\frac{u_{n}}{2}\left(L_{b}^{2}-2 a b+a^{2}\right)+\frac{u_{n}}{2}\left(L_{b}^{2}-2 a L_{b}-2 b L_{b}+a^{2}+2 a b+b^{2}\right) \\
& R_{A}=\frac{u_{n} b}{L_{b}}\left(L_{b}-a-b / 2\right)
\end{aligned}
$$

To determine the initial slope, $\theta(0)$, the deflection, $v(x)$, at $x=L_{b}$ is arranged knowing that the deflection at the end of the beam is zero. 


$$
\begin{aligned}
\begin{aligned}
& v\left(L_{b}\right)=0= \theta(0) L_{b} \\
&+\frac{1}{E I}\left(\frac{-M_{p}}{2}\left\langle L_{b}-0\right\rangle^{2}+\frac{R_{A}}{6}\left\langle L_{b}-0\right\rangle^{3}-\frac{u_{n}}{24}\left\langle L_{b}-a\right\rangle^{4}\right. \\
&\left.+\frac{u_{n}}{24}\left\langle L_{b}-(a+b)\right\rangle^{4}+\frac{R_{B}}{6}\left\langle L_{b}-L_{b}\right\rangle^{3}-\frac{M_{p}}{2}\left\langle L_{b}-L_{b}\right\rangle^{2}\right) \\
& \theta(0)=\frac{-1}{E I L_{b}}\left(\frac{-M_{p}}{2} L_{b}^{2}+\frac{R_{A}}{6} L_{b}^{3}-\frac{u_{n}}{24}\left(L_{b}-a\right)^{4}+\frac{u_{n}}{24}\left(L_{b}-a-b\right)^{4}\right)
\end{aligned}
\end{aligned}
$$

Now with the reaction force at the beginning of the beam known, the plastic bending moment across the beam can be broken up and modelled with piecewise functions.

$\mathbf{0} \leq \boldsymbol{x} \leq \boldsymbol{a}$

$$
\begin{aligned}
& M_{1}(x)=-M_{p}+R_{A} x \\
& M_{1}(x)=-M_{p}+u_{n} b x-\frac{u_{n} a b x}{L_{b}}-\frac{u_{n} b^{2} x}{2 L_{b}}
\end{aligned}
$$

$\boldsymbol{a} \leq \boldsymbol{x} \leq \boldsymbol{a}+\boldsymbol{b}$

$$
\begin{gathered}
M_{2}(x)=-M_{p}+R_{A} x-\frac{u_{n}}{2}(x-a)^{2} \\
M_{2}(x)=-M_{p}+u_{n} b x-\frac{u_{n} a b x}{L_{b}}-\frac{u_{n} b^{2} x}{2 L_{b}}-\frac{u_{n} x^{2}}{2}+u_{n} a x-\frac{u_{n} a^{2}}{2} \\
\boldsymbol{a}+\boldsymbol{b} \leq \boldsymbol{x} \leq \boldsymbol{L}_{\boldsymbol{b}} \\
M_{3}(x)=-M_{p}+R_{A} x-\frac{u_{n}}{2}(x-a)^{2}+\frac{u_{n}}{2}(x-(a+b))^{2} \\
M_{3}(x)=-M_{p}-\frac{u_{n} a b x}{L_{b}}-\frac{u_{n} b^{2} x}{2 L_{b}}+u_{n} a b-\frac{u_{n} b^{2}}{2}
\end{gathered}
$$




\section{Appendix D - Beam Strain Energy Integration (MATLAB Script)}

\section{Contents}

- Elastic Response

- $\quad$ Plastic Response

- $\underline{\text { Solve }}$

- Show Energy Equations

clear

syms Mp a b Lb u x

\section{Elastic Response}

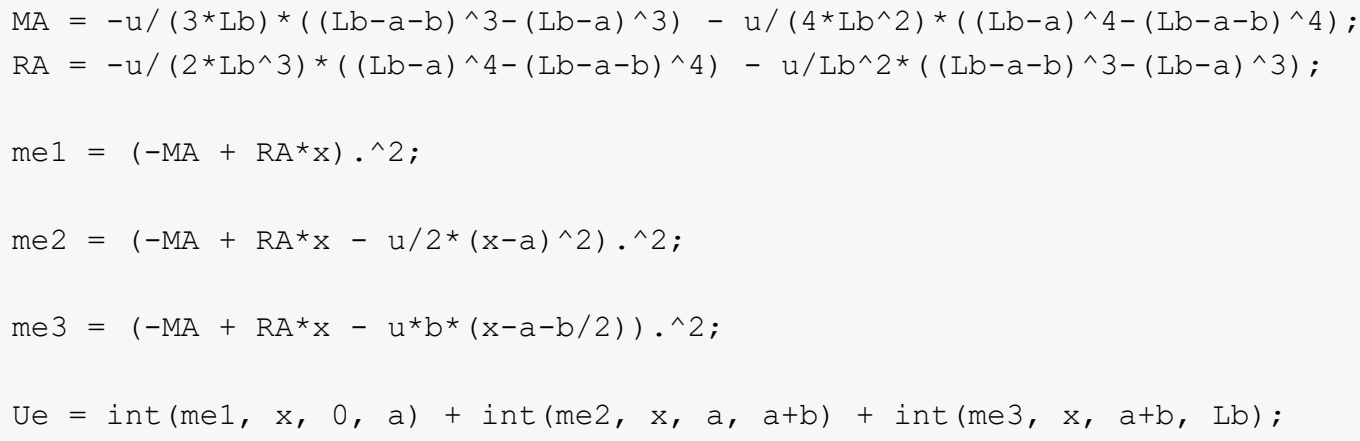

\section{Plastic Response}

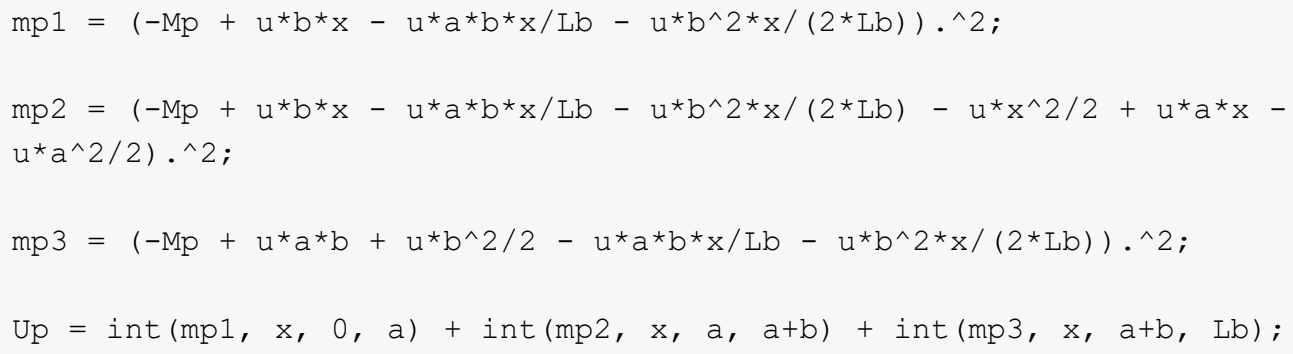

\section{Solve}

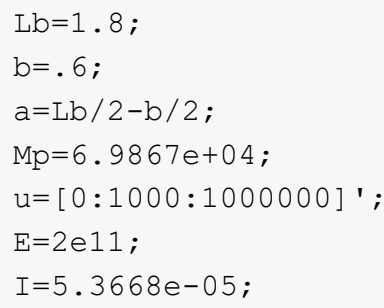




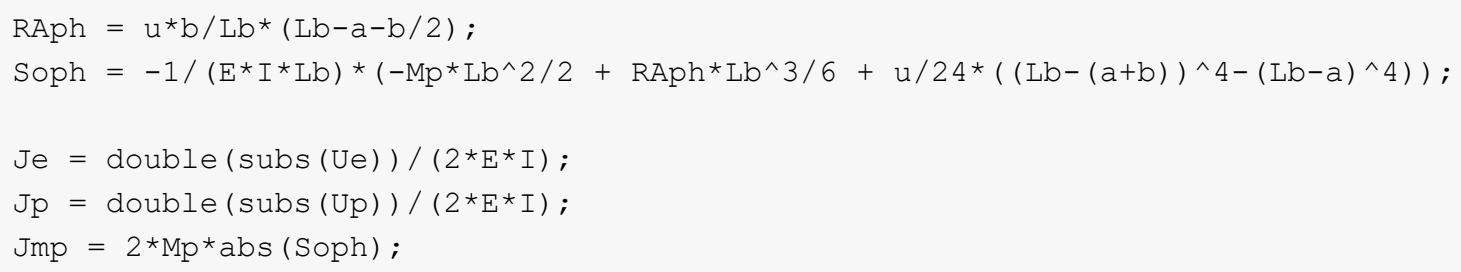

\section{Show Energy Equations}

displayFormula ("Ueb== Ue") displayFormula ("Upb== Up")

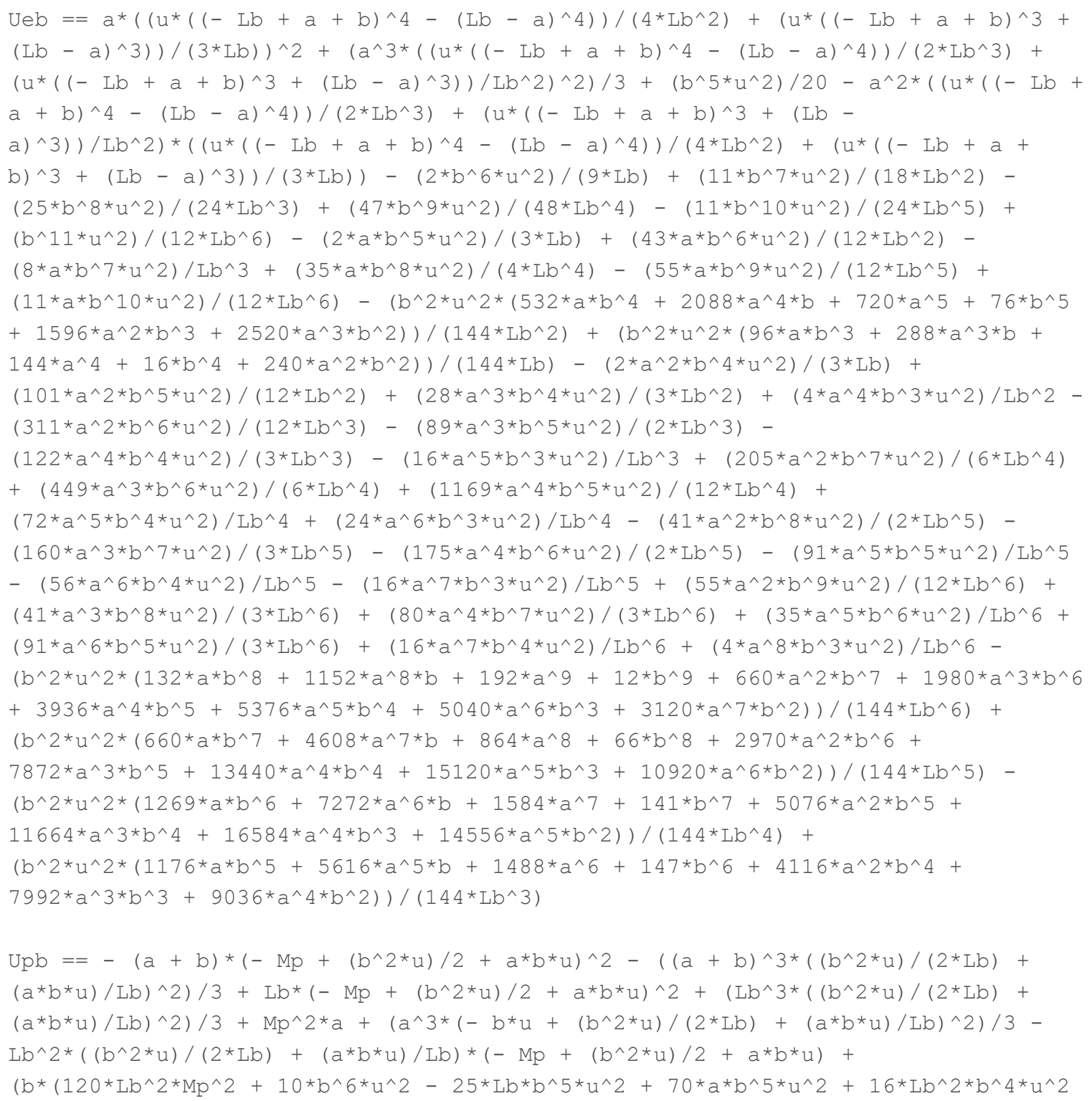




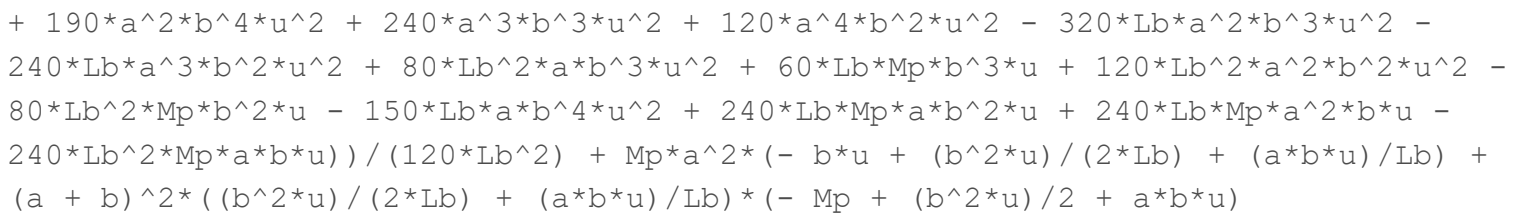

Published with MATLAB® R2019b 